\title{
ADIABATIC LIMITS, NONMULTIPLICATIVITY OF SIGNATURE, AND LERAY SPECTRAL SEQUENCE
}

\author{
XIANZHE DAI
}

\section{INTRODUCTION}

The $\eta$-invariant is introduced by Atiyah-Patodi-Singer [APS] as the correction term (from the boundary) for the index formula on a manifold with boundary. In this paper we study the limiting behavior of the $\eta$-invariant of a Dirac operator on the total space of a fibration, when the metric along the base direction is multiplied by a factor $x^{-2}$ and $x \rightarrow 0$. This operation of blowing up the metric is called passing to the adiabatic limit. The situation arises when one considers an open manifold. The usual passage from compact manifolds with boundary to open manifolds dictates that we study the contribution from the boundaries of an exhaustion. Additional algebraic structure on the open manifold often gives rise to a fibration structure on such boundaries, cf. [ADS, Mü2].

The original motivation, however, comes from a paper of E. Witten [W] where the adiabatic limit of the $\eta$-invariant is related to the so-called global anomaly. Witten's result was given full mathematical treatment in [BF1, BF2, $\mathrm{C} 2$ ], see also [Si].

Recently J.-M. Bismut and J. Cheeger, [BC1, BC2], extended the results of these papers to the case in which the base of the fibration is a compact spin manifold of arbitrary dimension. (Whereas the base in [W, BF1, BF2, C2] is a circle.) What they found is that the adiabatic limit of the $\eta$-invariant of a Dirac operator on the total space is expressible in terms of a canonically constructed differential form, $\tilde{\eta}$, on the base. This form $\tilde{\eta}$ can be viewed as a higher dimensional analogue of the $\eta$-invariant in the sense that it is exactly the boundary correction term in the Families Index Theorem for manifolds with boundary, [BC3, BC4].

In their work, they discussed in detail the case when the Dirac operators along the fibers are always invertible. Their treatment of the general case (i.e., the kernels of these operators have constant dimension) was less complete and

Received by the editors August 3, 1990.

1980 Mathematics Subject Classification (1985 Revision). Primary 58GXX; Secondary 35J05, 35K05, 35P15, 55R20, 55T10.

Supported in part by Alfred P. Sloan Doctoral Dissertation Fellowship. 
explicit although it did suffice for various applications. For example, one can recover the work of Atiyah-Donnelly-Singer [ADS] on the Hirzebruch conjecture, and prove results closely related to those of W. Müller [Mü1, Mü2] and M. Stern [S].

We study questions related to the noninvertible case, which is essential when we consider the signature operator because then the kernel is always nonempty and is given by the cohomology. Here some new phenomena occur. In particular, a global contribution arises from the (asymptotically) very small eigenvalues (cf. Theorem 0.1). What is more, in the case of the signature operator, these eigenvalues have a purely topological significance. In fact, the Leray spectral sequence can be recast in terms of these eigenvalues (Theorem 0.2). This is reminiscent of Hodge theory. Hence the term Hodge-Leray theory. As a consequence, we are able to identify the global contribution in the adiabatic limit formula with a topological invariant coming from the Leray spectral sequence of the fibration.

As an interesting application we give intrinsic characterization of the nonmultiplicativity of signature (see below) in terms of the Leray spectral sequence.

More precisely, let us consider a fibration of closed manifolds $Y \rightarrow M^{2 k-1} \stackrel{\pi}{\rightarrow}$ $B$. Equip M with a submersion metric $g_{M}$,

$$
g_{M}=\pi^{*} g_{B}+g_{Y},
$$

where $g_{B}$ is a metric on $B$ and $g_{Y}$ annihilates the orthogonal compliment of the tangent space to the fibers. Blowing up the metric in the horizontal direction by a factor $x^{-2}$ gives us a family of metrics $g_{x}$,

$$
g_{x}=x^{-2} \pi^{*} g_{B}+g_{Y} .
$$

We assume that $M$ is spin so that we can consider the Dirac operator $D_{x}$ associated with $g_{x}$. Further the bundle of vertical spaces $T^{V} M$ is also spin so that we have the family of Dirac operators $D_{Y}$ along the fibers.

It follows that the base space $B$ is also spin and the Dirac operator $D_{B}$ on the base is well defined. When $\operatorname{ker} D_{Y}$ is a vector bundle on $B$, one can couple $D_{B}$ to it to obtain a twisted Dirac operator $D_{B} \otimes \operatorname{ker} D_{Y}$. Here the connection on $\operatorname{ker} D_{Y}$ is the projection of a unitary connection on the infinite-dimensional bundle of smooth spinor sections along the fibers (cf. $\S 1.1$ ). Its curvature is denoted by $R_{0}$.

More generally, we consider the operator $D_{x}$ twisted by a hermitian vector bundle $\xi$ on $M$. Here $\xi$ is endowed with a unitary connection whose curvature is denoted by $L^{\xi}$. We suppress the $\xi$ dependence and use the same notation for the twisted Dirac operator. Let $\eta\left(D_{x}\right)$ be the $\eta$-invariant of $D_{x}$. Also let $R^{B}$ denote the curvature of the Levi-Civita connection of $g_{B}$ and $R^{Y}$ the curvature of the connection on $T^{V} M$ obtained by the projection of the Levi- 
Civita connection of $g_{M}$. Our first main result is

Theorem 0.1 (Adiabatic limit formula). Assume that $\operatorname{ker} D_{Y}$ is a vector bundle on $B$. Assume further that $\operatorname{dim}\left(\operatorname{ker} D_{x}\right)$ becomes constant when $0<x \leq x_{0}$ for some small $x_{0}$. Then $\lim _{x \rightarrow 0} \eta\left(D_{x}\right)$ exists in $\mathbb{R}$ and

$$
\lim _{x \rightarrow 0} \eta\left(D_{x}\right)=2 \int_{B} \widehat{A}\left(\frac{R^{B}}{2 \pi}\right) \wedge \widetilde{\eta}+\eta\left(D_{B} \otimes \operatorname{ker} D_{Y}\right)+\lim _{x \rightarrow 0} \sum_{\lambda_{0}, \lambda_{1}=0} \operatorname{sgn} \lambda_{x},
$$

where the summation $\sum_{\lambda_{0}, \lambda_{1}=0}$ runs over all eigenvalues $\lambda_{x}$ of $D_{x}$ decaying at least quadratically in $x$ (see Theorem 1.5). Moreover,

$$
d \widetilde{\eta}= \begin{cases}\int_{Y} \widehat{A}\left(\frac{R^{Y}}{2 \pi}\right) \wedge \operatorname{tr}\left(e^{-L^{\xi} / 2 \pi i}\right)-\operatorname{tr}_{s}\left(e^{-R_{0} / 2 \pi i}\right) & \text { if } \operatorname{dim} Y \text { even }, \\ \int_{Y} \hat{A}\left(\frac{R^{Y}}{2 \pi}\right) \wedge \operatorname{tr}\left(e^{-L^{\xi} / 2 \pi i}\right) & \text { if } \operatorname{dim} Y \text { odd }\end{cases}
$$

Remark 1. The summation $\sum_{\lambda_{0}, \lambda_{1}=0}$ is a finite sum (see Theorem 1.5; also below). Its limit exists because $\operatorname{dim}\left(\operatorname{ker} D_{x}\right)$ is assumed to stabilize eventually.

Remark 2. Formula (0.2) holds without the assumption on $\operatorname{dim} \operatorname{ker} D_{x}$. When $\operatorname{dim} Y$ is even, (0.2) should be viewed as a transgression formula. In fact, by the Chern-Weil theory, $\operatorname{tr}_{s}\left(e^{-R_{0} / 2 \pi i}\right)$ is a representative of $\operatorname{ch}\left(\operatorname{Ind} D_{Y}^{+}\right)$, the chern character of the index bundle for $D_{Y}^{+}$. On the other hand, Bismut's local family index theorem states that $\int_{Y} \widehat{A}\left(\frac{R^{Y}}{2 \pi}\right) \wedge \operatorname{tr}\left(e^{-L^{\xi} / 2 \pi i}\right)$ is also a representative of $\operatorname{ch}\left(\right.$ Ind $D_{Y}^{+}$). Thus $\widetilde{\eta}$ serves as a canonically constructed transgression between the two. When $\operatorname{dim} Y$ is odd, the assumption that $\operatorname{ker} D_{Y}$ gives a vector bundle clearly implies that there is no spectral flow for $D_{Y}$. It is a result of [BF2] that $\int_{Y} \widehat{A}\left(\frac{R^{Y}}{2 \pi}\right) \wedge \operatorname{tr}\left(e^{-L^{\xi} / 2 \pi i}\right)$ is a representative for the odd chern class of $D_{Y}$. Hence $(0.2)$ can be interpreted as saying that the higher spectral flow also vanishes.

When $D_{Y}$ is invertible, the spectrum of $D_{x}$ is uniformly bounded away from 0 , and the last two terms in (0.1) drop out. Thus formula (0.1) reduces to Bismut-Cheeger's adiabatic limit formula [BC2]. In general, there are infinitely many eigenvalues of $D_{x}$ approaching 0 as $x \downarrow 0$. This is due to the fact that the operator $D_{x}$ is degenerate elliptic and is in fact one of the essential difficulties we have to cope with in the noninvertible case.

In a recent work [MM], R. Mazzeo and R. Melrose studied the Laplacian in the adiabatic limit. Using a general theory of pseudodifferential calculus developed by R. Melrose et al. and well adapted to degenerate problems, they were able to construct a uniform parametrix and obtain a quite explicit description of the singularities of Green's operator. We use the same construction to treat the resolvent of $\frac{1}{x} D_{x}$ and analyze the behavior of the eigenvalues of $D_{x}$ as 
$x \downarrow 0$. We show that (Theorem 1.5) the spectrum of $D_{x}$ can be divided into three parts: those uniformly bounded away from 0 , those decaying linearly in $x$ (the small eigenvalues), and those, finitely many in number, decaying at least quadratically (the very small ones). Roughly speaking, the twisted $\eta$ term in $(0.1)$ comes from the small eigenvalues, and the last term from the very small ones (i.e. the finite sum of their signs).

Whereas asking $\operatorname{dim}\left(\operatorname{ker} D_{x}\right)$ to stabilize is a bit unnatural, it guarantees the existence of the adiabatic limit of the $\eta$-invariant, which does not happen in general. On the other hand, the limit of the reduced $\eta$-invariant, $\bar{\eta}$, always exists in $\mathbb{R} / \mathbb{Z}[\mathrm{C} 2, \mathrm{BC} 2]$. Hence, we have the following modulo $\mathbb{Z}$ counterpart of Theorem 0.1 .

Theorem 0.1'. Assume that $\operatorname{ker} D_{Y}$ is a vector bundle on $B$. Then

$$
\lim _{x \rightarrow 0} \bar{\eta}\left(D_{x}\right) \equiv \int \hat{A}\left(\frac{R^{B}}{2 \pi}\right) \wedge \tilde{\eta}+\bar{\eta}\left(D_{B} \otimes \operatorname{ker} D_{Y}\right)+\frac{1}{2} h \bmod \mathbb{Z},
$$

where $\frac{1}{2} h \equiv \frac{1}{2} \operatorname{dim}\left(\operatorname{ker} D_{x}\right)-\frac{1}{2} \operatorname{dim} \operatorname{ker}\left(D_{B} \otimes \operatorname{ker} D_{y}\right) \bmod \mathbb{Z}$ is a spin cobordism invariant [AS3].

An important operator satisfying the hypothesis of Theorem 0.1 is the socalled signature operator $A$, whose kernel space is identified with the cohomology by the Hodge theory. In this case, as we have indicated, the global contribution turns out to be a topological invariant. This is a consequence of the following interesting result.

Theorem 0.2. Let $E_{r}=$ the limit of the spaces spanned by the $\lambda_{x}$-eigenforms associated to eigenvalues $\lambda_{x}$ such that $\lambda_{x}$ is $O\left(x^{r}\right)(r \geq 2)$ in the adiabatic limit; then $\left(E_{r}, x^{-r} d\right)$ forms a spectral sequence that is isomorphic to the Leray spectral sequence of the fibration. Moreover, the * map induced by the metric $g_{x}$ gives rise to the duality map.

Remark. That such $E_{r}$ 's are well defined is a nontrivial result; compare Theorem (15) in [MM]. In [CD] we take a different point of view and study the adiabatic limit of a filtered differential complex. We prove a somewhat stronger version of Theorem 0.2 , and use it to study the $L^{2}$-cohomology of a cone bundle.

Theorem 0.2 gives us a refined adiabatic limit formula in the case of the signature operator. Let $\left(E_{r}, d_{r}\right)(r \geq 2)$ be the $E_{r}$-term of the Leray spectral sequence of the fibration $Y \rightarrow M^{4 k-1} \rightarrow B$. The orientation gives a natural basis $\xi_{2}$ on $E_{2}^{4 k-1}$ (in the sense of Chern-Hirzebruch-Serre [CHS], see $\S 4.3$ ), which then induces a basis $\xi_{r}$ on $E_{r}^{4 k-1}$ for each $r>2$. Consider the pairing

$$
\begin{aligned}
&\langle,\rangle_{r}: E_{r}^{p} \otimes E_{r}^{q} \longrightarrow \mathbb{R}, \\
& \varphi \otimes \psi \longrightarrow\left(\varphi \cdot d_{r} \psi, \xi_{r}\right) .
\end{aligned}
$$

Clearly $\langle,\rangle_{r}$ is symmetric when restricted to $E_{r}^{2 k-1}$. Therefore it gives rise to a symmetric matrix whose signature is denoted by $\tau_{r}$. Define $\tau=\sum_{r \geq 2} \tau_{r}$. 
Theorem 0.3. Suppose the fibration $Y \rightarrow M^{4 k-1} \rightarrow B$ is oriented (see $\S 4.1$ ), then

$$
\lim _{x \rightarrow 0} \eta\left(A_{x}\right)=2 \int_{B} \mathscr{L}\left(\frac{R^{B}}{2 \pi}\right) \wedge \tilde{\eta}+\eta\left(A_{B} \otimes \operatorname{ker} A_{Y}\right)+2 \tau,
$$

where $A_{B}$ denotes the signature operator on $B$ and $A_{Y}$ the family of signature operators along $Y$ (for the precise definition of the operator $A_{B} \otimes \operatorname{ker} A_{Y}$, see $\S 4.1)$.

Theorem 0.3 can be used to study the multiplicative behavior of the signature, in the following sense. Consider an oriented fibration $Z \rightarrow N^{4 k} \rightarrow B$ of compact manifolds (with or without boundary). Let $\operatorname{Sign}^{Z}$ denote the signature bundle of fibers on $B$. This virtual bundle can be considered as a $\mathbb{Z}_{2}$-graded bundle. It has a natural flat structure. Therefore we can define $\operatorname{sign}\left(B, \operatorname{Sign}^{Z}\right)$, the signature of $B$ with coefficients in the $\mathbb{Z}_{2}$-graded flat bundle $\operatorname{Sign}^{Z}$ (see [L]). Let

$$
\Delta \stackrel{\text { def }}{=} \operatorname{sign}\left(B, \operatorname{Sign}^{Z}\right)-\operatorname{sign}(N) .
$$

This is clearly a topological invariant of the fibration $Z \rightarrow N \rightarrow B$. We call it the nonmultiplicativity of signature. It measures the deviation of signature from being multiplicative. In the case of an oriented fibration of closed manifolds $Z \rightarrow N \rightarrow B$, when $\pi_{1}(B)$ acts trivially on $H^{*}(Z)$, the classical ChernHirzebruch-Serre Theorem [CHS] says

$$
\operatorname{sign} N=\operatorname{sign} B \operatorname{sign} Z \text {. }
$$

In general, the signature for closed oriented manifolds is multiplicative in the sense that

$$
\operatorname{sign} N=\operatorname{sign}\left(B, \operatorname{Sign}^{Z}\right) .
$$

This follows from the Atiyah-Singer index theorems and the signature theorem for twisted coefficients; see [A, AS1, L].

For manifolds with boundary, however, this does not hold generally, as examples show. Hence $\Delta \neq 0$ in general. (But compare [BC5], where it is shown that the index theory is asymptotically multiplicative in the invertible case.) Therefore it would be interesting to compute this nontrivial invariant. The boundary of the total space $N$ can arise either from the boundary of the fiber or from that of the base. For the simplicity of exposition, we restrict our attention to the case when $B$ is closed and $\partial Z=Y, \partial N=M$. (The same result holds for the other case, i.e. the base has boundary while the fiber is closed, see $\S 4.3$.) In this case, $\Delta$ depends only on the boundary fibration $Y \rightarrow M \rightarrow B$ by virtue of the extended Novikov additivity (cf. [BC5]). We have

Theorem 0.4 (intrinsic characterization of the nonmultiplicativity). The topological invariant $\tau$, which is defined from the closed fibration $Y \rightarrow M \rightarrow B$, intrinsically characterizes the nonmultiplicativity of signature. That is, whenever there exists another fibration of manifolds with boundary $Z \rightarrow N^{n} \rightarrow B$ such that $\partial Z=Y, \partial N=M$, then $\tau=\Delta$. 
Remark. It turns out that the invariant $\tau$ also computes the $L^{2}$-signature of the associated cone bundle, see [CD].

As another application of Theorem 0.3 , let us consider a locally symmetric space $X$ of $\mathbb{Q}$-rank one. The cross section of a cusp is a flat bundle over a compact locally symmetric space, cf. [Mü2]. In this case the invariant $\tau$ can be shown to be zero (see $\S 4.3$ ). Let $Y$ be the manifold with boundary obtained by chopping off all the cusps of $X$. Using the results of Müller [Mü2] and Theorem 0.3 , we can show that

$$
L^{2}-\operatorname{sign} X=\operatorname{sign}(Y, \partial Y) .
$$

Roughly speaking, the above suggests that the contributions from the cohomology at infinity cancel out. This is quite similar to [APS], where they showed that the $L^{2}$-signature of an elongation of a manifold with boundary is the same as the signature of the original manifold with boundary.

The remainder of the paper is organized as follows:

1. The adiabatic limit of the $\eta$-invariant

1.1. The local geometry of a fibration, the Levi-Civita superconnection and the $\tilde{\eta}$-form

1.2. Formal computation and outline of the proof

1.3. Large time behavior of heat kernels

1.4. Small time convergence

2. Asymptotic behavior of the spectrum

2.1. Analysis of degenerate elliptic operators

2.2. Asymptotic behavior of the spectrum

3. The uniform asymptotic expansion

3.1. Localization

3.2. A "rough" parametrix

4. The signature operator and Hodge-Leray theory

4.1. The signature operator on an odd-dimensional manifold

4.2. Hodge-Leray theory of the very small eigenvalues

4.3. Adiabatic limit of the $\eta$-invariant and nonmultiplicativity of signature

\section{THE ADIABATIC LIMIT OF THE $\eta$-INVARIANT}

By the work of Bismut-Cheeger the study of the adiabatic limit of the $\eta$ invariant of $D_{x}$ is essentially reduced to the study of the large time behavior of the heat kernel of $D_{x}$. In this section we analyze this large time behavior via Theorem 1.5, which gives detailed information about the spectrum of $D_{x}$ in the adiabatic limit. Theorem 1.5 is proved in the next section. We begin in $\S 1.1$, with some preliminaries and a brief review of the work of [BC2]. Then we present a formal computation, indicating some ideas behind the proof of Theorem 0.1. The justification for it is given in $\S \S 1.3$ and 1.4. 
1.1. The local geometry of a fibration, the Levi-Civita superconnection, and the $\tilde{\eta}$-form. Let

$$
Y \rightarrow M^{n} \stackrel{\pi}{\rightarrow} B^{p}
$$

be a smooth fibration of closed manifolds. A connection determines a splitting of the tangent bundle of $M$ into its vertical subbundle and horizontal subbundle,

$$
T M=T^{V} M \oplus T^{H} M
$$

Let $P^{H}, P^{V}$ denote the projections on $T^{H}, T^{V}$ respectively relative to this splitting. We use $U$ 's to denote the vertical vectors on $M$ and $X$ 's the tangent vectors on $B$ as well as their horizontal lifts to $M$.

Now equip $M$ with a submersion metric $g$, which preserves the splitting (1.2),

$$
g=\pi^{*} g_{B}+g_{Y}
$$

The Levi-Civita connection on $M$ is denoted by $\nabla^{L}$. This connection does not preserve the splitting (1.2). In [B] a unitary connection $\nabla$ is defined that respects the splitting. In fact,

$$
\begin{gathered}
\nabla_{U_{1}} U_{2}=P^{V}\left(\nabla_{U_{1}}^{L} U_{2}\right), \quad \nabla_{X} U=P^{V}\left(\nabla_{X}^{L} U\right), \\
\nabla_{U} X=0, \quad \nabla_{X_{1}} X_{2}=\nabla_{X_{1}}^{B} X_{2},
\end{gathered}
$$

where $\nabla^{B}$ denotes the Levi-Civita connection on $B$. Denote by $T$ the torsion tensor of $\nabla$ and $S$ the difference tensor of $\nabla^{L}$ and $\nabla$. Correspondingly, one has $\nabla^{L, x}$ and $S^{x}$ for $g_{x}$ ( $\nabla$ is invariant under this partial scaling). It can be verified that $[\mathrm{BC} 2, \S 4(\mathrm{a})]$

$$
\lim _{x \rightarrow 0} \nabla^{L, x}=\nabla+P^{V} S
$$

and is in uppertriangular form (in terms of the splitting (1.2)) with the diagonal entry $\nabla$. Consequently, its curvature is also in uppertriangular form with the diagonal entry $R=\nabla^{2}$. From this we deduce

Lemma 1.1. Let $P$ be an $O(n)$-invariant polynomial on the Lie algebra $o(n)$, and $R^{x}, R=R^{Y} \oplus R^{B}$ the curvatures of $\nabla^{L, x}, \nabla$ respectively. Then

$$
\lim _{x \rightarrow 0} P\left(R^{x} / 2 \pi i\right)=P(R / 2 \pi i)=P\left(R^{Y} / 2 \pi i\right) P\left(R^{B} / 2 \pi i\right) .
$$

Assume from now on that $n(=\operatorname{dim} M)$ is odd. Further, assume that both $M$ and $T^{V} M$ are spin. Consequently, so is $B$. By [BC2, 11$]$, the spinor bundle $F(M)$ of the total space is related to $F(B)$, the spinor bundle of the base, and 
$F(Y)$, the spinor bundle of $T^{V} M$, in the following way:

$$
F(M)=\pi^{*} F(B) \otimes F(Y) \text {. }
$$

Let $\xi$ be a hermitian bundle on $M$ with unitary connection $\nabla^{\xi}$ and curvature $L^{\xi}$, and let $\nabla^{u}$ denote the connection on $F(M) \otimes \xi$ defined by

$$
\nabla^{u}=\nabla-\frac{1}{2} \sum\left\langle S\left(e_{i}\right) e_{i}, \cdot\right\rangle,
$$

where $\left\{e_{i}\right\}$ is a local orthonormal basis for $T^{V} M$. The Dirac operator along the fiber $D_{Y}$ is defined as $D_{Y}=e_{i} \nabla_{e_{i}}$. We have the following nice formula for $D_{x}[\mathrm{BC} 2,(4.26)]$.

Lemma 1.2. If $\left\{f_{\alpha}\right\}$ is a local orthonormal basis for $T B$, then

$$
D_{x}=x \sum_{\alpha} f_{\alpha} \nabla_{f_{\alpha}}^{u}+D_{Y}-\frac{x^{2}}{4} \sum_{\alpha \leq \beta} f_{\alpha} f_{\beta} T\left(f_{\alpha}, f_{\beta}\right) \text {. }
$$

For simplicity, we denote $\widetilde{D}_{B}=\sum_{\alpha} f_{\alpha} \nabla_{f_{\alpha}}^{u}, T=\sum_{\alpha \leq \beta} f_{\alpha} f_{\beta} T\left(f_{\alpha}, f_{\beta}\right)$.

We now recall the definition of the Levi-Civita superconnection introduced by Bismut in [B]. The smooth sections of $F(Y) \otimes \xi$ can be viewed as the smooth sections of an infinite-dimensional vector bundle $H_{\infty}$ over $B$. The fiber of $H_{\infty}$ over a point $y \in B$ is the space of smooth sections of $\left.(F(Y) \otimes \xi)\right|_{\pi^{-1}(y)}$. Thus it comes with a natural $L^{2}$-metric. The connection $\nabla^{u}$ induces a unitary connection $\tilde{\nabla}^{u}$ on $H_{\infty}$. If we wish to regard the operator $D_{x}$ as acting on sections of $F(B) \otimes H_{\infty}$, we simply write $\tilde{\nabla}^{u}$ for $\nabla^{u}$ in (1.3). The Levi-Civita superconnection $B_{t}$ on $H_{\infty}$ is the superconnection

$$
B_{t}=\tilde{\nabla}^{u}+t^{1 / 2} D_{Y}-c(T) / 4 t^{1 / 2}
$$

where $c(T)=\sum_{\alpha \leq \beta} d y^{\alpha} d y^{\beta} T\left(f_{\alpha}, f_{\beta}\right)$, and $d y^{\alpha}$ denotes the 1 -form dual to $f_{\alpha}$. It follows from (1.3) that $\frac{1}{x} D_{x}$ is the Dirac operator coupled to the LeviCivita superconnection $B_{x^{-2}}$, cf. [BC2, Appendix 1].

The asymptotics of heat kernels associated to the Levi-Civita superconnection exhibit some remarkable cancellations. The first one is expressed in the local index theorem for families [B, BF2]. Essential to our discussion are the other two cancellation results [BC2]; i.e. when $\operatorname{dim} Y=2 l$ is even

$$
\operatorname{tr}_{s}\left[\left(D_{Y}+c(T) / 4 t\right) e^{-B_{t}^{2}}\right]=O\left(t^{1 / 2}\right) \text { as } t \rightarrow 0,
$$

or when $\operatorname{dim} Y=2 l-1$ is odd

$$
\operatorname{tr}^{\text {even }}\left[\left(D_{Y}+c(T) / 4 t\right) e^{-B_{t}^{2}}\right]=O\left(t^{1 / 2}\right) \text { as } t \rightarrow 0
$$

where $\operatorname{tr}^{\text {even }}$ indicates taking the even form part of $\operatorname{tr}$.

Remarkably, when $\operatorname{ker} D_{Y}$ is a vector bundle on $B$, the expressions on the left-hand sides of (1.5), (1.6) are also well behaved for the large time. In fact, 
it is shown in [BGV] (in a more general setting) that for $\operatorname{dim} Y=2 l$,

$$
\operatorname{tr}_{s}\left[\left(D_{Y}+c(T) / 4 t\right) e^{-B_{t}^{2}}\right]=O\left(t^{-1}\right) \text { as } t \rightarrow \infty
$$

and for $\operatorname{dim} Y=2 l-1$,

$$
\operatorname{tr}^{\text {even }}\left[\left(D_{Y}+c(T) / 4 t\right) e^{-B_{t}^{2}}\right]=O\left(t^{-1}\right) \text { as } t \rightarrow \infty .
$$

By virtue of (1.5)-(1.8), we now define a differential form on $B$, the $\widehat{\eta}$ form

$$
\widehat{\eta}= \begin{cases}\frac{1}{\sqrt{\pi}} \int_{0}^{\infty} \operatorname{tr}_{s}\left[\left(D_{Y}+\frac{c(T)}{4 t}\right) e^{-B_{t}^{2}}\right] \frac{d t}{2 t^{1 / 2}} & \text { if } \operatorname{dim} Y=2 l, \\ \frac{1}{\sqrt{\pi}} \int_{0}^{\infty} \operatorname{tr}^{\text {even }}\left[\left(D_{Y}+\frac{c(T)}{4 t}\right) e^{-B_{t}^{2}}\right] \frac{d t}{2 t^{1 / 2}} & \text { if } \operatorname{dim} Y=2 l-1 .\end{cases}
$$

For example, the first integral is convergent at 0 because of $(1.5)$, and convergent at $\infty$ because of (1.7).

The $\hat{\eta}$ should be viewed as a higher dimensional analogue of the $\eta$-invariant. In fact, when $\operatorname{dim} Y=2 l-1$ is odd, its 0 -form component is exactly the $\eta$ invariant of the Dirac operator along the fiber. Moreover, results of [BC3, $\mathrm{BC} 4]$ show that it is precisely the boundary correction term in the Families Index Theorem for manifolds with boundary. We also point out that when $\operatorname{dim} Y=2 l$ the 1 -form component of $\hat{\eta}$ represents the Quillen connection of the determinant line bundle $\operatorname{det} D_{Y}^{+}$, which is interpreted by Witten as the covariant anomaly $[\mathrm{W}]$; see also $[\mathrm{BF} 2, \mathrm{C} 2, \mathrm{~F}]$.

We normalize $\hat{\eta}$ by defining

$$
\tilde{\eta}= \begin{cases}\sum \frac{1}{(2 \pi i)^{j}}[\widehat{\eta}]_{2 j-1} & \text { if } \operatorname{dim} Y=2 l, \\ \sum \frac{1}{(2 \pi i)^{j}}[\widehat{\eta}]_{2 j} & \text { if } \operatorname{dim} Y=2 l-1 .\end{cases}
$$

Here we decompose the odd (respectively even) form $\hat{\eta}$ into its homogeneous components $[\hat{\eta}]_{2 j-1}$ (respectively $[\widehat{\eta}]_{2 j}$ ).

Theorem 1.3 (Bismut-Cheeger). Assume that $D_{Y}$, the Dirac operator along the fiber, is always invertible. Then the limit $\lim _{x \rightarrow 0} \bar{\eta}\left(D_{x}\right)=\lim _{x \rightarrow 0} \frac{1}{2} \eta\left(D_{x}\right)$ exists in $\mathbb{R}$ and

$$
\lim _{x \rightarrow 0} \bar{\eta}\left(D_{x}\right)=\int_{B} \widehat{A}\left(\frac{R^{B}}{2 \pi}\right) \wedge \tilde{\eta} .
$$

Moreover,

$$
d \widetilde{\eta}=\int_{Y} \widehat{A}\left(\frac{R^{Z}}{2 \pi}\right) \wedge \operatorname{tr}\left(e^{-L^{\xi} / 2 \pi i}\right) .
$$


We briefly recall the basic ideas involved in the proof. By virtue of the local regularity result of [BF2], the $\eta$-invariant of $D_{x}$ has a heat kernel representation:

$$
\eta\left(D_{x}\right)=\frac{1}{\sqrt{\pi}} \int_{0}^{+\infty} t^{-1 / 2} \operatorname{tr}\left(D_{x} e^{-t D_{x}^{2}}\right) d t .
$$

Note that this involves the contribution from the heat kernel for all the time. For $t \in(0, T]$, we have uniform convergence as $x \rightarrow 0$ (in the case of odddimensional fiber, the other case similar),

$$
\operatorname{tr}\left(D_{x} e^{-D_{x}^{2} t}\right)=\frac{\sqrt{\pi}}{(2 \pi i)^{k}} \int_{B} \widehat{A}\left(i R^{B}\right) \operatorname{tr}^{\text {even }}\left(\left(D_{Y}+\frac{c(T)}{4 t}\right) e^{-B_{t}^{2}}\right)+O\left(x\left(1+T^{N}\right)\right)
$$

for some $N$. This is shown in [BC2, $\S 4]$ by exploitation of the so-called Getzler's transformation.

For large time the heat kernel contribution is negligible as $x \rightarrow 0$. This is because when $D_{Y}$ is invertible, there is a uniform lower bound for the smallest eigenvalues of $D_{x}^{2}$, see [BC2, $\left.\S 4\right]$ for the details.

1.2. Formal computation and outline of the proof. Our starting point is the following observation, which is a direct consequence of the proof of Theorem 1.3 (cf. (1.9) above).

Proposition 1.4. Without the assumption that $D_{Y}$ is invertible, one can still find a small positive number $\alpha$ such that

$$
\lim _{x \rightarrow 0} \eta\left(D_{x}\right)=2 \int_{B} \widehat{A}\left(\frac{R^{B}}{2 \pi}\right) \wedge \tilde{\eta}+\lim _{x \rightarrow 0} \frac{1}{\sqrt{\pi}} \int_{x^{-\alpha}}^{\infty} t^{-1 / 2} \operatorname{tr}\left(D_{x} e^{-D_{x}^{2} t}\right) d t,
$$

provided either one of the limits exists.

Here the second term is the large time contribution alluded to previously. The discussion on the invertible case indicates that information about the spectrum of $D_{x}$ in the adiabatic limit is crucial in understanding the large time behavior of the heat kernel. In this respect, we have the following result, the proof of which is deferred to $\S 2$.

Theorem 1.5. For $x>0$ the eigenvalues of $D_{x}$ depend analytically on $x$. Thus there are (countably many) analytic functions $\lambda_{x}$ such that $\operatorname{spec}\left(D_{x}\right)=\left\{\lambda_{x}\right\}$ for all $x>0$. Moreover, when $\operatorname{ker} D_{Y}$ is a vector bundle on $B$ :

(A) asymptotic behavior. There exists a positive constant $\lambda_{0}$ such that either $\lambda_{x}$ is uniformly bounded away from 0 by $\lambda_{0}$,

$$
\left|\lambda_{x}\right| \geq \lambda_{0}>0
$$

or $\lambda_{x}$ has a complete asymptotic expansion as $x \rightarrow 0$,

$$
\lambda_{x} \sim \lambda_{1} x+\lambda_{2} x^{2}+\cdots
$$


where $\lambda_{1} \in \operatorname{spec}\left(D_{B} \otimes \operatorname{ker} D_{Y}\right)$. Here the connection on $\operatorname{ker} D_{Y}$ is given by projecting the connection $\widetilde{\nabla}^{u}$ on $\operatorname{ker} D_{Y}$. In the latter case, the correspondence $\lambda_{x} \leftrightarrow \lambda_{1}$ is bijective (counting multiplicity);

(B) uniform remainder estimate. If $\lambda_{x}$ corresponds to $\lambda_{1}$ and $\lambda_{1} \neq 0$, then

$$
\lambda_{x}=\lambda_{1} x+x^{2} C(x) \lambda_{1}^{2},
$$

with $|C(x)| \leq C$ uniformly bounded;

(C) finiteness. For any $K>0$,

$$
\#\left\{\lambda_{x} \mid \lambda_{x} \sim \lambda_{1} x+\cdots \text { and }\left|\lambda_{1}\right| \leq K\right\}<+\infty .
$$

In particular, the number of eigenvalues with $\lambda_{1}=0$ (those of at least quadratic decay) is finite.

Granted Theorem 1.5, the generalized adiabatic limit formula (Theorem 0.1) can be seen formally as follows. By making a substitution (or rescaling)

$$
\begin{aligned}
\lim _{x \rightarrow 0} & \int_{x^{-\alpha}}^{+\infty} t^{-1 / 2} \operatorname{tr}\left(D_{x} e^{-t D_{x}^{2}}\right) d t=\lim _{x \rightarrow 0} \int_{x^{2-\alpha}}^{+\infty} t^{-1 / 2} \operatorname{tr}\left(\frac{1}{x} D_{x} e^{-t\left(\frac{1}{x} D_{x}\right)^{2}}\right) d t \\
= & \lim _{x \rightarrow 0}\left(\int_{x^{2-\alpha}}^{+\infty} t^{-1 / 2} \sum_{\substack{\left|\lambda_{x}\right| \geq \lambda_{0} \\
x}} \frac{1}{x} \lambda_{x} e^{-t\left(\frac{1}{x} \lambda_{x}\right)^{2}} d t\right) \\
& +\lim _{x \rightarrow 0}\left(\int_{x^{2-\alpha}}^{+\infty} t^{-1 / 2} \sum_{\substack{\lambda_{x} \sim \lambda_{1} x+\cdots \\
\lambda_{1} \neq 0}} \frac{1}{x} \lambda_{x} e^{-t\left(\frac{1}{x} \lambda_{x}\right)^{2}} d t\right) \\
& +\lim _{x \rightarrow 0}\left(\int_{x^{2-\alpha}}^{+\infty} t^{-1 / 2} \sum_{\lambda_{x} \sim \lambda_{2} x^{2}+\cdots} \frac{1}{x} \lambda_{x} e^{-t\left(\frac{1}{x} \lambda_{x}\right)^{2}} d t\right) \\
= & \mathrm{I}+\mathrm{II}+\mathrm{III} .
\end{aligned}
$$

In I note that for $t \in\left[x^{2-\alpha},+\infty\right)$ and $\left|\lambda_{x}\right| \geq \lambda_{0}, t\left(\frac{1}{x} \lambda_{x}\right)^{2} \geq \lambda_{0}^{2} x^{-\alpha}$. Therefore, $\lim _{x \rightarrow 0} \frac{1}{x} \lambda_{x} e^{-t\left(\frac{1}{x} \lambda_{x}\right)^{2}}=0$.

For II we have $\frac{1}{x} \lambda_{x} \rightarrow \lambda_{1}$ for $\lambda_{1} \in \operatorname{spec}\left(D_{B} \otimes \operatorname{ker} D_{Y}\right)$. Thus

$$
\lim _{x \rightarrow 0} \frac{1}{x} \lambda_{x} e^{-t\left(\frac{1}{x} \lambda_{x}\right)^{2}}=\lambda_{1} e^{-t \lambda_{1}^{2}} .
$$

Finally for III, we use Mellin's formula to get

$$
\int_{x^{2-\alpha}}^{+\infty} t^{-1 / 2} \frac{1}{x} \lambda_{x} e^{-t\left(\frac{1}{x} \lambda_{x}\right)^{2}} d t=\operatorname{sgn}\left(\frac{1}{x} \lambda_{x}\right) \int_{x^{2-\alpha}}^{\infty} u^{-1 / 2} e^{-u} d u .
$$

Hence, modulo technical problems arising from infinite sums,

$$
\begin{aligned}
\lim _{x \rightarrow 0} \int_{x^{-\alpha}}^{+\infty} t^{-1 / 2} \operatorname{tr}\left(D_{x} e^{-t D_{x}^{2}}\right) d t= & 0+\int_{0}^{+\infty} t^{-1 / 2} \operatorname{tr}\left[\left(D_{B} \otimes \operatorname{ker} D_{Y}\right) e^{-t\left(D_{B} \otimes \operatorname{ker} D_{Y}\right)^{2}}\right) d t \\
& +\lim _{x \rightarrow 0} \sqrt{\pi} \sum_{\lambda_{x} \sim \lambda_{2} x^{2}+\cdots} \operatorname{sgn} \lambda_{x},
\end{aligned}
$$


and consequently, Proposition 1.4 yields

$$
\lim _{x \rightarrow 0} \eta\left(D_{x}\right)=2 \int_{B} \hat{A}\left(\frac{R^{B}}{2 \pi}\right) \wedge \tilde{\eta}+\eta\left(D_{B} \otimes \operatorname{ker} D_{Y}\right)+\lim _{x \rightarrow 0} \sum_{\lambda_{x} \sim \lambda_{2} x^{2}+\cdots} \operatorname{sgn} \lambda_{x} .
$$

The above formal calculation suggests the following.

(1) Those eigenvalues that are uniformly bounded away from 0 do not contribute.

(2) Those eigenvalues that decay exactly linearly in $x$ give rise to the twisted $\eta$-invariant on the base.

(3) The finiteness result in Theorem 1.5 justifies the calculation of III.

The justification of (1) involves estimates by the finite propagation speed technique [CGT], while that of (2) is a consequence of Theorem 1.5. In $\S 1.3$ combining (1) and (2), we prove a stronger result. We show that the heat kernel on the total space under the metric shrinking of the fibers converges to the heat kernel on the base.

Remark 1. Notice that after rescaling, the time interval for integration in (1.2) is $\left[x^{2-\alpha}, \infty\right)$. So there arises the question of small time convergence. This is dealt with in $\S 1.4$.

Remark 2. Intuitively, when we are in the finite time, the effect of blowing up is to localize on the base. Hence the semilocal term $2 \int_{B} \widehat{A} \wedge \tilde{\eta}$. When time goes like $x^{-2}$, which is comparable to the speed at which the base is blowing up, one starts to pick up some global contributions. This is the twisted $\eta$ term. After $x^{-4}$, one picks up some "very" global information, i.e. the very small eigenvalues. In [DM], using the notion of blow up in [M2], the above picture can be made very precise, thus giving an essentially complete description of the heat kernel in the adiabatic limit. The discussion generalizes to manifolds with boundary as well.

1.3. Large time behavior of heat kernels. Motivated by the above formal calculation, we rescale the (large) time interval $\left[x^{-\alpha}, \infty\right)$ back to $\left[x^{2-\alpha}, \infty\right)$,

$$
\int_{x^{-\alpha}}^{+\infty} t^{-1 / 2} \operatorname{tr}\left(D_{x} e^{-t D_{x}^{2}}\right) d t=\int_{x^{2-\alpha}}^{+\infty} t^{-1 / 2} \operatorname{tr}\left(\frac{1}{x} D_{x} e^{-t\left(\frac{1}{x} D_{x}\right)^{2}}\right) d t
$$

This rescaling corresponds to the metric rescaling

$$
x^{2} g_{x}=\pi^{*} g_{B}+x^{2} g_{Y}
$$

which shrinks the metric along the fiber direction. Our first step toward proving the adiabatic limit formula is the following result, which appears to have an interest of its own. 
Theorem 1.6. Let $D_{0}=D_{B} \otimes \operatorname{ker} D_{Y}$. Then for $N=p+1+\frac{n+5}{2}$ and $x$ small

$$
\left|\operatorname{tr}^{\prime}\left(\frac{1}{x} D_{x} e^{-t\left(\frac{1}{x} D_{x}\right)^{2}}\right)-\operatorname{tr}\left(D_{0} e^{-t D_{0}^{2}}\right)\right| \leq \frac{C x}{t^{N}} e^{-C^{\prime} t}
$$

where $\operatorname{tr}^{\prime}$ indicates taking trace over those eigenvalues, which decay at most linearly in $x$. Here and throughout, the $C$ 's are constants that depend on the dimensions and geometry of the manifolds considered but not on $t$ and $x$.

Taking into account the rest of the eigenvalues (i.e., those decaying quadratically) by using Theorem 1.5, we can rewrite the result in Theorem 1.6 in a nicer although weaker form,

$$
\left|\operatorname{tr}\left(\frac{1}{x} D_{x} e^{-t\left(\frac{1}{x} D_{x}\right)^{2}}\right)-\operatorname{tr}\left(D_{0} e^{-t D_{0}^{2}}\right)\right| \leq\left(\frac{C}{t^{N}}+C^{\prime}\right) x .
$$

Roughly speaking, this formula says that when the total space collapses to the base (in the sense of Cheeger-Gromov) its heat kernels converge to the corresponding heat kernels on the base.

The proof of Theorem 1.6 goes exactly as our formal calculation suggested, except that we take a different dividing line between the large eigenvalues (those not contributing) and small eigenvalues (those giving rise to the twisted $\eta$ term). This is determined by the uniform remainder estimate of Theorem 1.5. It turns out that workable dividing lines are $\lambda_{0} x^{a}$ for any $0<a<1$. We take $a=1 / 2$.

Proof. One has

$$
\operatorname{tr}^{\prime}\left(\frac{1}{x} D_{x} e^{-t\left(\frac{1}{x} D_{x}\right)^{2}}\right)=\sum^{\prime} \frac{1}{x} \lambda_{x} e^{-t\left(\frac{1}{x} \lambda_{x}\right)^{2}},
$$

where the summation $\Sigma^{\prime}$ runs over all (nonzero) eigenvalues of $D_{x}$ that decay at most linearly. Also

$$
\operatorname{tr}\left(D_{0} e^{-t D_{0}^{2}}\right)=\sum \lambda_{1} e^{-t \lambda_{1}^{2}}
$$

where the summation runs over all (nonzero) eigenvalues of $D_{0}$. Thus

$$
\begin{aligned}
\mid \operatorname{tr}^{\prime}( & \left.\frac{1}{x} D_{x} e^{-t\left(\frac{1}{x} D_{x}\right)^{2}}\right)-\operatorname{tr}\left(D_{0} e^{-t D_{0}^{2}}\right) \mid \\
\quad & \left|\sum^{\prime} \frac{1}{x} \lambda_{x} e^{-t\left(\frac{1}{x} \lambda_{x}\right)^{2}}-\sum \lambda_{1} e^{-t \lambda_{1}^{2}}\right| \\
& \leq\left|\sum_{\left|\lambda_{x}\right| \geq \lambda_{0} \sqrt{x}} \frac{1}{x} \lambda_{x} e^{-t\left(\frac{1}{x} \lambda_{x}\right)^{2}}\right|+\left|\sum^{\prime}\right| \lambda_{x}\left|<\lambda_{0} \sqrt{x} \frac{1}{x} \lambda_{x} e^{-t\left(\frac{1}{x} \lambda_{x}\right)^{2}}-\sum \lambda_{1} e^{-t \lambda_{1}^{2}}\right| .
\end{aligned}
$$

We now divide our discussion into two parts.

(1) Large eigenvalues. Define

$$
f_{x}(\lambda)=\left\{\begin{array}{ll}
\lambda e^{-t \lambda^{2}} & \text { if }|\lambda| \geq \lambda_{0} / \sqrt{x}, \\
0 & \text { otherwise; }
\end{array} \quad h_{x}(\lambda)= \begin{cases}\lambda^{2} e^{-t \lambda^{2}} & \text { if }|\lambda| \geq \lambda_{0} / \sqrt{x} \\
0 & \text { otherwise }\end{cases}\right.
$$


Then the operators $f_{x}\left(\frac{1}{x} D_{x}\right)$ and $h_{x}\left(\frac{1}{x} D_{x}\right)$ can be defined by the spectral theorem. Moreover, by the spectral mapping theorem,

$$
\begin{aligned}
\left|\operatorname{tr}\left(f_{x}\left(\frac{1}{x} D_{x}\right)\right)\right| & =\left|\sum_{\left|\lambda_{x} / x\right| \geq \lambda_{0} / \sqrt{x}} \frac{1}{x} \lambda_{x} e^{-t\left(\frac{1}{x} \lambda_{x}\right)^{2}}\right| \\
& \leq \sum_{\left|\lambda_{x} / x\right| \geq \lambda_{0} / \sqrt{x}}\left|\frac{1}{x} \lambda_{x}\right| e^{-t\left(\frac{1}{x} \lambda_{x}\right)^{2}} \\
& \leq \sum_{\left|\lambda_{x} / x\right| \geq \lambda_{0} / \sqrt{x}}\left(\frac{1}{x} \lambda_{x}\right)^{2} e^{-t\left(\frac{1}{x} \lambda_{x}\right)^{2}}=\operatorname{tr}\left(h_{x}\left(\frac{1}{x} D_{x}\right)\right),
\end{aligned}
$$

for $x$ small enough. Choose a nonnegative function $\varphi_{x}(\lambda) \in C^{\infty}(-\infty,+\infty)$ such that $\varphi_{x}(\lambda) \equiv 0$ for $|\lambda| \leq \lambda_{0} / 2 \sqrt{x}$ and $\varphi_{x}(\lambda) \equiv 1$ for $|\lambda| \geq \lambda_{0} / \sqrt{x}$. Further $\left\|\varphi_{x}\right\|_{C^{2}} \leq C$ uniformly for $x \in(0,1]$. Set

$$
H=H_{x}(\lambda)=\lambda^{2} e^{-t \lambda^{2}} \varphi_{x}(\lambda)
$$

Clearly we have

$$
\operatorname{tr}\left(h_{x}\left(\frac{1}{x} D_{x}\right)\right) \leq \operatorname{tr}\left(H_{x}\left(\frac{1}{x} D_{x}\right)\right) .
$$

Let $k_{H_{x}}=k_{H_{x}\left(\frac{1}{x} D_{x}\right)}$ denote the (Schwartz) kernel of the operator $H_{x}\left(\frac{1}{x} D_{x}\right)$ (with respect to the volume element induced by the metric $x^{2} g_{x}$ ). We want to estimate $k_{H_{x}}$ by the finite propagation speed technique of Cheeger-GromovTaylor [CGT].

First of all, note that $\frac{1}{x} D_{x}$ corresponds to the metric $x^{2} g_{x}$. The sectional curvature of this metric is bounded by $C\left(1 / x^{2}\right)$, and the injectivity radius is bounded from below by $C x$ (cf. [C2]). Therefore, by (1.29) of [CGT], one has the pointwise estimate

$$
\left|k_{H_{x}}\right| \leq \frac{C}{x^{n+1}} \sum_{k=0}^{n+1} \int_{0}^{+\infty}\left|\widehat{H}^{(k)}(s)\right| d s,
$$

where $n=\operatorname{dim} M$. Thus it remains to estimate $\int_{0}^{+\infty}\left|\widehat{H}^{(k)}(s)\right| d s$. To do this, write

$$
\int_{0}^{+\infty}\left|\widehat{H}^{(k)}(s)\right| d s=\int_{0}^{+\infty} \frac{1}{\left(1+s^{2}\right)}\left(1+s^{2}\right)\left|\widehat{H}^{(k)}(s)\right| d s .
$$

We show that

$$
\left|\left(1+s^{2}\right) \widehat{H}^{(k)}(s)\right| \leq \frac{C(k)}{t^{(n+5) / 2}} e^{-t \lambda_{0} / 4 x} .
$$


By calculus and the basic properties of the Fourier transform, we have

$$
\begin{aligned}
\left(1+s^{2}\right) \widehat{H}^{(k)}(s) & =\widehat{H}^{(k)}(s)+s^{2} \widehat{H}^{(k)}(s) \\
& =\widehat{H}^{(k)}(s)+\left(s^{2} \widehat{H}(s)\right)^{(k)}-2 k(s \widehat{H})^{(k-1)}-(k-1)^{2} \widehat{H}^{(k-1)}(s) \\
& \left.=\widehat{H}^{(k)}(s)+\widehat{H}^{\prime \prime}(s)\right)^{(k)}-2 k\left(\widehat{H}^{\prime}\right)^{(k-1)}-(k-1)^{2} \widehat{H}^{(k-1)}(s) .
\end{aligned}
$$

Now

$$
\begin{aligned}
\left|\widehat{H}^{(k)}(s)\right| & =\left|\int e^{-i s \lambda}(-i \lambda)^{k} H(\lambda) d \lambda\right| \\
& \leq C \int_{|\lambda| \geq \lambda_{0} / 2 \sqrt{x}}\left|\lambda^{k+2} e^{-t \lambda^{2}}\right| d \lambda \\
& \leq 2 \int_{\lambda_{0} / 2 \sqrt{x}}^{\infty} \frac{C(k)}{t^{(k+1) / 2}} \lambda e^{-t \lambda^{2} / 2} d \lambda=\frac{C(k)}{t^{(k+1) / 2}} e^{-t \lambda_{0}^{2} / 4 x} .
\end{aligned}
$$

The last step comes from the elementary inequality

$$
\lambda^{2 j} e^{-t \lambda^{2}} \leq t^{-j} C(j) e^{-t \lambda^{2} / 2}
$$

One can apply the same argument as above to estimate $\left(\widehat{H}^{\prime}\right)^{(k-1)}$ and $\left.\widehat{\left(H^{\prime \prime}\right.}\right)^{(k)}$. Plug $(1.18)$ and the corresponding estimates for $\left({\widehat{H^{\prime}}}^{(k-1)}\right.$ and $\left(\widehat{H^{\prime \prime}}\right)^{(k)}$ in (1.17), and together with (1.15), (1.16), one finds

$$
\left|k_{H_{x}\left(\frac{1}{x} D_{x}\right)}\right| \leq \frac{C}{x^{n+1} t^{(n+5) / 2}} e^{-t \lambda_{0}^{2} / 4 x} .
$$

Thus one can integrate to obtain

$$
\operatorname{tr}\left(H_{x}\left(\frac{1}{x} D_{x}\right)\right) \leq \frac{C}{x^{p+1} t^{(n+5) / 2}} e^{-t \lambda_{0}^{2} / 4 x},
$$

where $p$ is the dimension of the base $B$. Consequently,

$$
\left|\sum_{\left|\lambda_{x} / x\right|>\lambda_{0} / \sqrt{x}} \frac{1}{x} \lambda_{x} e^{-t\left(\frac{1}{x} \lambda_{x}\right)^{2}}\right| \leq \frac{C}{x^{p+1} t^{(n+5) / 2}} e^{-t \lambda_{0}^{2} / 4 x} \leq \frac{C\left(p, \lambda_{0}\right) x}{t^{p+2+(n+5) / 2}} e^{-t \lambda_{0}^{2} / 8 x},
$$

again by (1.19).

(2) Small eigenvalues. If $\left|\lambda_{x}\right|<\lambda_{0}$, then by Theorem 1.5, $\frac{1}{x} \lambda_{x} \rightarrow \lambda_{1}$ for some $\lambda_{1} \in \operatorname{spec}\left(D_{0}\right)$. Since we are taking $\operatorname{tr}^{\prime}$, we have $\lambda_{1} \neq 0$. Now

$$
\begin{aligned}
& \left|\sum_{\left|\lambda_{x}\right|<\lambda_{0} \sqrt{x}}^{\prime} \frac{1}{x} \lambda_{x} e^{-t\left(\frac{1}{x} \lambda_{x}\right)^{2}}-\sum \lambda_{1} e^{-t \lambda_{1}^{2}}\right| \\
& \leq\left|\sum_{\left|\lambda_{x}\right|<\lambda_{0} \sqrt{x}}^{\prime}\left(\frac{1}{x} \lambda_{x}-\lambda_{1}\right) e^{-t\left(\frac{1}{x} \lambda_{x}\right)^{2}}\right|+\left|\sum_{\left|\lambda_{x}\right|<\lambda_{0} \sqrt{x}}^{\prime} \lambda_{1}\left(e^{-t\left(\frac{1}{x} \lambda_{x}\right)^{2}}-e^{-t \lambda_{1}^{2}}\right)\right| \\
& +\left|\sum_{\left|\lambda_{1}\right| \geq \lambda_{0} / 2 \sqrt{x}} \lambda_{1} e^{-t \lambda_{1}^{2}}\right|=\mathrm{I}+\mathrm{II}+\mathrm{III} .
\end{aligned}
$$


By the same argument as we show (1.20) (in a simpler situation), it is easy to see that

$$
\text { III } \leq \frac{C}{t^{p / 2}} e^{-t \lambda_{0}^{2} / 4 x} \leq \frac{C x}{t^{p / 2+1}} e^{-t \lambda_{0}^{2} / 8 x} .
$$

To estimate I and II, we invoke the uniform remainder estimate in Theorem 1.5. Note that the remainder there is bounded by a uniform constant times the square of $\lambda_{1}$, which can be arbitrarily large. To cope with this problem, we now use the fact that the summations are over $\left|\lambda_{x}\right|<\lambda_{0} \sqrt{x}$, together with the estimates (2.12), (2.13) in the proof of Theorem 1.5, to show that $\lambda_{1}$ cannot grow too fast relative to $x$.

Since $\frac{1}{x} \lambda_{x} \rightarrow \lambda_{1}$, by taking $x \rightarrow 0$ in (2.12) where $\bar{\lambda}_{x}$ obeys (2.13), we deduce

$$
\left|\lambda_{1}-\lambda_{0}^{\prime}\right| \leq C
$$

Now $\left|\lambda_{x}\right|<\lambda_{0} \sqrt{x}$ together with (2.13) and (2.12) yields

$$
\left|\lambda_{0}^{\prime}\right| \leq\left|\bar{\lambda}_{x}\right|+C x \leq\left|\frac{1}{x} \lambda_{x}\right|+2 C \leq \frac{\lambda_{0}}{\sqrt{x}}+2 C \leq \frac{2 \lambda_{0}}{\sqrt{x}} .
$$

Combining the two gives

$$
\left|\lambda_{1}\right| \leq 3 \lambda_{0} / \sqrt{x}
$$

Hence $\lambda_{1}$ grows no faster than $x^{-1 / 2}$. It follows from this and the uniform remainder estimate that

$$
\left(\frac{1}{x} \lambda_{x}\right)^{2}=\lambda_{1}^{2}\left(1+x C(x) \lambda_{1}\right)^{2} \geq \frac{\lambda_{1}^{2}}{4}
$$

provided $x \leq 1 / 36 \lambda_{0}^{2} C^{2}$. This combined with (1.11) implies

$$
\begin{aligned}
\mathrm{I} & \leq \sum_{\lambda_{1} \neq 0} x C \lambda_{1}^{2} e^{-t\left(\frac{1}{x} \lambda_{x}\right)^{2}} \leq x C \sum_{\lambda_{1} \neq 0} \lambda_{1}^{2} e^{-t \lambda_{1}^{2} / 4} \\
& \leq \frac{C x}{t^{n / 2+1}} e^{-C^{\prime} t} .
\end{aligned}
$$

The last step is by virtue of (1.19) and the standard heat kernel estimates (cf., say, [CGT]).

For II, notice that by using the inequality $\left|e^{-\lambda}-1\right| \leq|\lambda| e^{|\lambda|}$ (which follows by the standard expansion),

$$
\begin{aligned}
\left|e^{-t\left(\frac{1}{x} \lambda_{x}\right)^{2}}-e^{-t \lambda_{1}^{2}}\right| & =\left|e^{-t \lambda_{1}^{2}}\left(e^{-t\left[\left(\frac{1}{x} \lambda_{x}\right)^{2}-\lambda_{1}^{2}\right]}-1\right)\right| \\
& \leq t C \lambda_{1}^{4} x e^{-t \lambda_{1}^{2}\left(1-2 C x\left|\lambda_{1}\right|-C^{2} x^{2} \lambda_{1}^{2}\right)} .
\end{aligned}
$$

Therefore,

$$
\mathrm{II} \leq C x t \sum\left|\lambda_{1}\right|^{3} e^{-t \lambda_{1}^{2} / 4} \leq \frac{C x}{t^{n / 2+1}} e^{-C^{\prime} t} .
$$

These estimates combined with (1.20) give us (1.13). 
Remark. The above method allows a more general statement. For example, if $f \in \mathscr{S}([0,+\infty))$ is in the Schwartz class, then the same argument (with a slight adaptation) gives an estimate of the same type for $\left|\operatorname{tr}^{\prime}\left(f\left(\frac{1}{x} D_{x}\right)\right)-\operatorname{tr}^{\prime}\left(f\left(D_{0}\right)\right)\right|$. Here the first $\operatorname{tr}^{\prime}$ means the same as before, and the second $\operatorname{tr}^{\prime}$ indicates taking out the zero spectrum. An interesting example of such an $f$ would be $e^{-t \lambda^{2}}$, which gives rise to the heat kernel. Thus

$$
\left|\operatorname{tr}^{\prime}\left(e^{-t\left(\frac{1}{x} D_{x}\right)^{2}}\right)-\operatorname{tr}^{\prime}\left(e^{-t D_{0}^{2}}\right)\right| \leq \frac{C x}{t^{N}} e^{-C^{\prime} t} .
$$

As an immediate consequence of Theorem 1.6, we have

Corollary 1.6. There exists $\beta>0$ sufficiently small such that

$$
\begin{aligned}
\lim _{x \rightarrow 0} & \frac{1}{\sqrt{\pi}} \int_{C x^{-2+\beta}}^{+\infty} t^{-1 / 2} \operatorname{tr}\left(D_{x} e^{-t D_{x}^{2}}\right) d t \\
& =\frac{1}{\sqrt{\pi}} \int_{0}^{+\infty} t^{-1 / 2} \operatorname{tr}\left(D_{0} e^{-t D_{0}^{2}}\right) d t+\lim _{x \rightarrow 0} \sum_{\lambda_{x} \sim \lambda_{2} x^{2}+\cdots} \operatorname{sgn} \lambda_{x},
\end{aligned}
$$

if either one of the limits exists in $\mathbb{R}$.

Proof.

$$
\begin{aligned}
\lim _{x \rightarrow 0} & \frac{1}{\sqrt{\pi}} \int_{C x^{-2+\beta}}^{+\infty} t^{-1 / 2} \operatorname{tr}\left(D_{x} e^{-t D_{x}^{2}}\right) d t \\
& =\lim _{x \rightarrow 0} \frac{1}{\sqrt{\pi}} \int_{C x^{\beta}}^{+\infty} t^{-1 / 2} \operatorname{tr}\left(\frac{1}{x} D_{x} e^{-t\left(\frac{1}{x} D_{x}\right)^{2}}\right) d t \\
& =\lim _{x \rightarrow 0} \frac{1}{\sqrt{\pi}} \int_{C x^{\beta}}^{+\infty} t^{-1 / 2} \operatorname{tr}^{\prime}\left(\frac{1}{x} D_{x} e^{-t\left(\frac{1}{x} D_{x}\right)^{2}}\right) d t+\lim _{x \rightarrow 0} \sum_{\lambda_{x} \sim \lambda_{2} x^{2}+\cdots} \operatorname{sgn} \lambda_{x} .
\end{aligned}
$$

In the last step above, we used Mellin's formula and the fact that the number of eigenvalues that decay at least quadratically is finite. Now take $0<\beta<1 / 2 N$ and apply Theorem 1.6.

1.4. Small time convergence. In the proceeding section large time behavior of the heat kernel is analyzed by virtue of our detailed knowledge of the (small) eigenvalues. As a result we obtained (1.22), which furnished a justification for (1) and (2) of $\S 1.2$, but only for the time interval $\left[x^{-2+\beta}, \infty\right)$, where $\beta$ is a sufficiently small positive number. Thus we are left with the task of showing that the remaining piece (the small time contribution after the rescaling) is negligible. This is done by establishing a uniform asymptotic expansion.

Theorem 1.7. One has the following uniform pointwise asymptotic expansion,

$$
\operatorname{tr}\left(D_{x} e^{-t D_{x}^{2}}\right)=\sum_{i=-n}^{N-1} a_{i}(t)\left(t x^{2}\right)^{i / 2}+O\left(\left(t x^{2}\right)^{N / 2}\right), \quad \text { as } x \rightarrow 0,
$$

where $a_{i}(t)$ 's are bounded for $t \geq 1$, and so is $O(\cdot)$. 
The proof of this theorem is given in $\S 3$. Here we demonstrate that Theorems 1.6 and 1.8 combined imply the small time convergence.

Proposition 1.8. For any $\beta>0$ small, $2>\alpha>0$,

$$
\lim _{x \rightarrow 0} \int_{x^{-\alpha}}^{x^{-2+\beta}} t^{-1 / 2} \operatorname{tr}\left(D_{x} e^{-t D_{x}^{2}}\right) d t=0
$$

Proof. That $\operatorname{tr}\left(D_{x} e^{-t D_{x}^{2}}\right)$ converges as $x \downarrow 0$ implies significant cancellation here. In fact, since for fixed $t$ the left-hand side of (1.23) approaches a finite limit as $x \rightarrow 0$, we must have

$$
a_{i}=0, \quad \text { if } i<0 .
$$

A further cancellation result is furnished by our uniform asymptotic expansion and estimate (1.14). Again,

$$
\lim _{x \rightarrow 0} \int_{x^{-\alpha}}^{x^{-2+\beta}} t^{-1 / 2} \operatorname{tr}\left(D_{x} e^{-t D_{x}^{2}}\right) d t=\lim _{x \rightarrow 0} \int_{x^{2-\alpha}}^{x^{\beta}} t^{-1 / 2} \operatorname{tr}\left(\frac{1}{x} D_{x} e^{-t\left(\frac{1}{x} D_{x}\right)^{2}}\right) d t .
$$

Now we apply the uniform asymptotic expansion (1.23) to $\operatorname{tr}\left(D_{x} e^{-t\left(\frac{1}{x} D_{x}\right)^{2}}\right)$ with $t / x^{2}$ as the new time parameter. Thus

$$
\operatorname{tr}\left(D_{x} e^{-t\left(\frac{1}{x} D_{x}\right)^{2}}\right)=\sum_{i=0}^{N^{\prime}-1} a_{i}\left(t / x^{2}\right) t^{i / 2}+O\left(t^{N^{\prime} / 2}\right)
$$

and hence

$$
\operatorname{tr}\left(\frac{1}{x} D_{x} e^{-t\left(\frac{1}{x} D_{x}\right)^{2}}\right)=\sum_{i=0}^{N^{\prime}-1} a_{i}\left(t / x^{2}\right) t^{i / 2} x^{-1}+O\left(t^{N^{\prime} / 2} x^{-1}\right)
$$

To obtain cancellation in the $x$ parameter, we use the estimate (1.14). Take $M \geq N+1, N^{\prime} \geq 2 M+1$ and set $x=t^{M} \rightarrow 0$. By the same reasoning as above one finds

$$
a_{i}\left(t^{1-2 M}\right) t^{-M+\frac{i}{2}}=O(1)
$$

Or

$$
a_{i}\left(t^{-1}\right)=O\left(t^{\frac{M}{2 M-1}-\frac{i}{2(2 M-1)}}\right) .
$$

Plugging in (1.25) we finally arrive at

$$
\operatorname{tr}\left(\frac{1}{x} D_{x} e^{-t\left(\frac{1}{x} D_{x}\right)^{2}}\right)=\sum_{i=0}^{N^{\prime}-1} a_{i}^{\prime} t^{(i-1) \frac{M}{2 M-1}} x^{-\frac{i-1}{2 M-1}}+O\left(t^{N^{\prime} / 2} / x^{-1}\right)
$$


where $a_{i}^{\prime}$ are bounded functions. Consequently

$$
\begin{aligned}
& \left|\int_{x^{2-\alpha}}^{x^{\beta}} t^{-1 / 2} \operatorname{tr}\left(\frac{1}{x} D_{x} e^{-t\left(\frac{1}{x} D_{x}\right)^{2}}\right) d t\right| \\
& \leq C x^{1 /(2 M-1)} \int_{x^{2-\alpha}}^{x^{\beta}} t^{-1 / 2-M /(2 M-1)} d t \\
& +\sum_{i=1}^{N^{\prime}-1} C x^{(i-1)(\alpha M-1) /(2 M-1)} \int_{x^{2-\alpha}}^{x^{\beta}} t^{-1 / 2} d t+C\left(x^{\beta}-x^{2-\alpha}\right) x^{\left(N^{\prime}-1\right) \alpha / 2-1} \\
& \leq C x^{\alpha /(4 M-2)}+\sum_{i=1}^{N^{\prime}-1} C x^{(i-1)(\alpha M-1) /(2 M-1)}\left(x^{\beta / 2}-x^{1-\alpha / 2}\right) \\
& +C\left(x^{\beta}-x^{2-\alpha}\right) x^{\left(N^{\prime}-1\right) \alpha / 2-1} \text {. }
\end{aligned}
$$

Taking $M \geq \alpha^{-1}$ and $N^{\prime} \geq 2 \alpha^{-1}+1$ gives us (1.24).

Proof of Theorem 0.1. By Proposition 1.4, Corollary 1.7, and Proposition 1.9, to show the existence of $\lim _{x \rightarrow 0} \eta\left(A_{x}\right)$ and the resulting formula for it, we just have to show the existence of $\lim _{x \rightarrow 0} \sum_{\lambda_{0}, \lambda_{1}=0} \operatorname{sgn} \lambda_{x}$. The only way it would not exist is if such a $\lambda_{x}$ fluctuates around 0 as $x \downarrow 0$. This is prevented by our assumption that $\operatorname{dim}\left(\operatorname{ker} D_{x}\right)$ stabilizes eventually.

As for the transgression formula, we prove it for $\operatorname{dim} Y$ odd since the discussions are parallel. Let us first note that (cf. [BC2, (4.38)])

$$
\begin{aligned}
\frac{\partial}{\partial t} \operatorname{tr}\left(e^{-B_{t}^{2}}\right) & =-d \operatorname{tr}\left(\frac{\partial B_{t}}{\partial t} e^{-B_{t}^{2}}\right) \\
& =-d \operatorname{tr}\left(\left(D_{Y}+\frac{c(T)}{4 t}\right) e^{-B_{t}^{2}}\right) \frac{1}{2 t^{1 / 2}} .
\end{aligned}
$$

Taking the odd form part, we have

$$
\frac{\partial}{\partial t} \operatorname{tr}^{\text {odd }}\left(e^{-B_{t}^{2}}\right)=-d \operatorname{tr}^{\text {even }}\left(\left(D_{Y}+\frac{c(T)}{4 t}\right) e^{-B_{t}^{2}}\right) \frac{1}{2 t^{1 / 2}} .
$$

Thus

$$
-d \hat{\eta}=\lim _{t \rightarrow \infty} \operatorname{tr}^{\text {odd }}\left(e^{-B_{t}^{2}}\right)-\lim _{t \rightarrow 0} \operatorname{tr}^{\text {odd }}\left(e^{-B_{t}^{2}}\right)
$$

Here $\hat{\eta}$ is the unnormalized version of $\tilde{\eta}$; see $\S 1.1$. It is shown in [BF2] that

$$
\lim _{t \rightarrow 0} \operatorname{tr}^{\text {odd }}\left(e^{-B_{t}^{2}}\right)=\int_{Y} \widehat{A}\left(i R^{Y}\right) \wedge \operatorname{tr}\left(e^{-L^{\xi}}\right) .
$$

On the other hand, when $\operatorname{ker} D_{Y}$ is a vector bundle, it is proved in [BGV] that

$$
\lim _{t \rightarrow \infty} e^{-B_{t}^{2}}=e^{-R_{0}}
$$

where $R_{0}$ is the curvature of the connection on $\operatorname{ker} D_{Y}$. Since $e^{-R_{0}}$ contains only even forms, our formula follows. 


\section{ASYMPTOTIC BEHAVIOR OF THE SPECTRUM}

This section is devoted to the proof of Theorem 1.5. Recall that $D_{x}$ is associated with the metric that is rescaled in the base direction by $x^{-2}$. From the local point of view, the rescaling is making the operator $D_{x}$ better since the local geometry is simplifying. And this is the reason that for the finite time behavior of the heat kernel one can work effectively with the so-called Getzler's transformation. However, from the global point of view, the elliptic operator $D_{x}$ becomes degenerate as $x \downarrow 0$. This degeneracy is the essential difficulty here.

Recently using a general theory of pseudodifferential calculus developed by Melrose et al. (cf. [M1-M3]), Mazzeo and Melrose [MM] constructed a uniform parametrix for the Laplacian in the adiabatic limit. From there they obtain a quite explicit description of the singularities of Green's operator. Their construction extends to Dirac operators as well. Our main point here is to use this description to show that the resolvents of the operators $\frac{1}{x} D_{x}$ are $L^{2}$-bounded and further as $L^{2}$-bounded operators these resolvents depend smoothly on the parameter $x$ down to $x=0$. The result, combined with the regular perturbation theory, gives us the asymptotic behavior of the small eigenvalues at $x=0$, in $\S 2.2$. The finiteness result, which is a consequence of ellipticity, is also discussed there. The uniform remainder estimate is established by a deformation argument where the local geometry of the fibration plays a definite role.

2.1. Analysis of degenerate elliptic operators. We first fix some notations. Throughout this section, for $Z$ a manifold with corner, $U \subset Z$ an embedded submanifold, and $E \rightarrow Z$ a vector bundle, $I^{m}(Z, U ; E)$ denotes the space of $E$-valued distributions conormal to $U$, of order $m$. The reader is referred to $[\mathrm{H}]$ for the basic properties of conormal distributions.

Let $M$ be the total space of the fibration (1.1). Set

$$
X=[0, \infty) \times M, \quad Z=[0, \infty) \times M \times M=X \times M .
$$

The spinor bundle $F(M)$ lifts to $X$ and $Z$ in the obvious way. The Schwartz kernels of the operators concerned here are generally distributions on $Z$. However, the degeneracy of our problem means that these distributions have complicated singularities. One of the central ideas in [MM] is the notion of blow up.

Let

$$
Q=\left\{\left(0, p, p^{\prime}\right) \mid \pi_{L}(p)=\pi_{R}\left(p^{\prime}\right)\right\} \subset Z=[0, \infty) \times M \times M,
$$

where $\pi_{L}$ and $\pi_{R}$ are the left and right fibrations. The blow up of $Z$ along the submanifold $Q$ is

$$
Z_{Q}=S N Q \sqcup[Z \backslash Q],
$$

where $S N Q$ is the inward-pointing unit spherical bundle of $Q$ in $Z$. This is a manifold with corner with two codimension 1 boundary faces and one 
codimension 2 boundary face. As a set it is given by replacing $Q$ by $S N Q$. It comes equipped with the 'blow-down' map

$$
\pi_{Q}: Z_{Q} \rightarrow Z
$$

which is the identity away from the 'front face' $S N Q$, which we denote $\operatorname{ff}\left(Z_{Q}\right)$. The blown up space $Z_{Q}$ has a unique $C^{\infty}$ structure such that $\pi_{Q}$ is a $C^{\infty}$ diffeomorphism from $Z_{Q} \backslash \mathrm{ff}\left(Z_{Q}\right)$ to $Z \backslash Q$ and has rank $\operatorname{dim} B+2 \operatorname{dim} Y+1$ at ff $\left(Z_{Q}\right)$. Moreover, the diagonal $\Delta=\{(x, p, p) \mid x \geq 0, p \in M\}$ has a unique lift $\Delta_{\mathscr{V}}$ to $Z_{Q}$, which intersects ff $\left(Z_{Q}\right)$ transversally; see [MM].

The significance of the blown up space $Z_{Q}$ is that the singularities of the parametrix and hence of the resolvents can be much better described when lifted up to $Z_{Q}$. Let $R$ be a defining function of the front face. Then $\rho=x / R$ is a defining function for the boundary face other than the front face. As we will see, besides the usual diagonal singularity the boundary singularity only involves the powers of these defining functions.

Following [MM], the Schwartz kernels here are taken with respect to the singular measure $d g_{x}=x^{-p} d g$. By a straightforward modification of the construction in [MM] (for the simplicity of notation, in what follows, we use "Hom" to denote "End $F(M)$ " and " $F$ " to denote "F(M)"), we have

Proposition 2.1. Assume that $\operatorname{ker} D_{Y}$ gives a vector bundle on $B$. If $\lambda \notin \operatorname{spec}\left(D_{0}\right)$, then the resolvent $\left(\frac{1}{x} D_{x}-\lambda\right)^{-1}$ exists for $x$ sufficiently small. Further, its Schwartz kernel lies in the space $x \mathscr{G}$ where

$$
\begin{aligned}
\mathscr{G}= & I_{0}^{-1}\left(Z_{Q}, \Delta_{\mathscr{V}} ; \text { Hom }\right)+\rho^{p-1} C^{\infty}\left(Z_{Q}, \text { Hom }\right)+x^{p-1} \log R \cdot C^{\infty}(Z, \text { Hom }) \\
& +x^{p-1} C^{\infty}(Z, \text { Hom })
\end{aligned}
$$

and it maps $C^{\infty}(X, F)$ into itself. Here $I_{o}^{-1}$ consists of all conormal distributions in $I^{-1}$ that vanish in a neighborhood of $\partial Z_{Q} \backslash f\left(Z_{Q}\right)$.

With this explicit description of the resolvent, we can now proceed to prove

Theorem 2.2. Every element of $x \mathscr{G}$ defines a bounded operator on $L^{2}(M, F)$ and consequently $\left(\frac{1}{x} D_{x}-\lambda\right)^{-1}\left(\lambda \notin \operatorname{spec}\left(D_{0}\right)\right)$ defines a family of bounded operators on $L^{2}(M, F)$. Furthermore, this family depends smoothly on $x$ down to $x=0$.

Proof. We divide the proof into two parts, corresponding to the two statements in the theorem.

(A) One shows that each element of

$$
\begin{array}{cl}
x I_{0}^{-1}\left(Z_{Q}, \Delta_{\mathscr{V}} ; \text { Hom }\right), & x \rho^{p-1} C^{\infty}\left(Z_{Q}, \text { Hom }\right), \\
x^{p} \log R \cdot C^{\infty}(Z, \text { Hom }), & x^{p} C^{\infty}(Z, \text { Hom })
\end{array}
$$

defines bounded operator on $L^{2}(M, F)$. This is clear for elements in $x^{p} C^{\infty}(Z$, Hom $)$. We now look at $x \rho^{p-1} C^{\infty}\left(Z_{Q}\right.$, Hom $)$. Let $y, z$ be local 
coordinate systems on $B$ and $Y$ respectively. Since we are using the singular measure $d g_{x}=x^{-p} d y d z$, if $G$ is a Schwartz kernel, then

$$
\begin{aligned}
(G \phi)(x, y, z) & =\int_{M} G\left(x, y, z, y^{\prime}, z^{\prime}\right) \phi\left(y^{\prime}, z^{\prime}\right) d g_{x} \\
& =\int_{M} x^{-p} G \phi d y^{\prime} d z^{\prime} .
\end{aligned}
$$

For $G \in x \rho^{p-1} C^{\infty}\left(Z_{Q}\right.$, Hom $)$,

$$
G^{\prime} \stackrel{\text { def }}{=} x^{-p} G \in x^{-p+1} \rho^{p-1} C^{\infty}\left(Z_{Q}, \text { Hom }\right)=R^{1-p} C^{\infty}\left(Z_{Q}, \text { Hom }\right) .
$$

If $x>0$, we have $R>0$ and $G^{\prime}$ clearly defines a $L^{2}$-bounded operator. Thus it suffices to prove the statement for $x=0$. By using the Schwarz inequality,

$$
\begin{aligned}
\int|(G \phi)(x, y, z)|^{2} d y d z= & \int\left|\int G^{\prime}\left(x, y, z, y^{\prime}, z^{\prime}\right) \phi\left(y^{\prime}, z^{\prime}\right) d y^{\prime} d z^{\prime}\right|^{2} d y d z \\
\leq & \int\left(\int\left|G^{\prime}\left(x, y, z, y^{\prime}, z^{\prime}\right)\right| d y^{\prime} d z^{\prime}\right) \\
& \times\left(\int\left|G^{\prime}\left(x, y, z, y^{\prime}, z^{\prime}\right)\right|\left|\phi\left(y^{\prime}, z^{\prime}\right)\right|^{2} d y^{\prime} d z^{\prime}\right) d y d z .
\end{aligned}
$$

Introduce polar coordinates around $Q$ :

$$
R=\left(x^{2}+\left|y-y^{\prime}\right|^{2}\right)^{1 / 2}, \quad \omega=\left(\frac{x}{R}, \frac{y-y^{\prime}}{R}\right)=\left(\rho, \omega^{\prime}\right), y, z, z^{\prime} .
$$

This defines a local coordinate near the front face on $Z_{Q}$. We can take our previous defining function $R$ and replace it by the above defined $R$ since they are both defining functions for the front face. Let

$$
\left|G^{\prime}\right|(R, \omega)=\sup _{z, y^{\prime}, z^{\prime}}\left|R^{p-1} G^{\prime}\left(R, \omega, z, y^{\prime}, z^{\prime}\right)\right| .
$$

This is a smooth function of $R$ and $\omega$. Therefore

$$
\begin{aligned}
\int\left|G^{\prime}\left(0, y, z, y^{\prime}, z^{\prime}\right)\right| d y^{\prime} d z^{\prime} & =\int\left|G^{\prime}\left(R, \omega, z, y^{\prime}, z\right)\right| R^{p-1} d R d \omega^{\prime} \\
& \leq \int\left|G^{\prime}\right|(R, \omega) d R d \omega^{\prime}=C^{\prime}<\infty
\end{aligned}
$$

Plug this into (2.1) to get

$$
\begin{aligned}
& \int|(G \phi)(0, y, z)|^{2} d y d z \\
& \leq C^{\prime} \iint\left|G^{\prime}\left(0, y, z, y^{\prime}, z^{\prime}\right)\right|\left|\phi\left(y^{\prime}, z^{\prime}\right)\right|^{2} d y^{\prime} d z^{\prime} d y d z \\
& \quad=C^{\prime} \int\left(\int\left|G^{\prime}\left(0, y, z, y^{\prime}, z^{\prime}\right)\right| d y d z\right)\left|\phi\left(y^{\prime}, z^{\prime}\right)\right|^{2} d y^{\prime} d z^{\prime} \\
& \quad \leq C^{\prime 2} \int\left|\phi\left(y^{\prime}, z^{\prime}\right)\right|^{2} d y^{\prime} d z^{\prime} .
\end{aligned}
$$


This is the desired $L^{2}$-boundedness of $x \rho^{p-1} C^{\infty}\left(Z_{Q}\right.$, Hom $)$. From the proof, we see that the singularity here comes from the factor $R^{1-p}$, which is cancelled in the polar coordinates. The argument clearly carries over for $x^{p} \log R \cdot C^{\infty}(Z$, Hom $)$.

For $x I_{0}^{-1}\left(Z_{Q}, \Delta_{\mathscr{V}} ;\right.$ Hom $)$, one reduces it to the $L^{2}$-boundedness of

$$
x I_{0}^{-\infty}\left(Z_{Q}, \Delta_{\mathscr{V}} ; \text { Hom }\right) \subset x C^{\infty}\left(Z_{Q}, \Delta_{\mathscr{V}} ; \text { Hom }\right) .
$$

Then, because the support of the elements of $x I_{0}^{-\infty}\left(Z_{Q}, \Delta_{\mathscr{V}}\right.$; Hom $)$ is contained in $\{\rho \neq 0\}$, the same argument as above works also for $x I_{0}^{-\infty}\left(Z_{Q}, \Delta_{\mathscr{V}} ;\right.$ Hom $)$. For the reduction we need the following version of Hörmander's lemma.

Lemma 2.3. If $A \in I^{0}\left(Z_{Q}, \Delta_{\mathscr{V}}\right.$; Hom $)$ and $\nu \in C^{\infty}(M, \Omega M)$ is a positive density on $M$, then there exists a constant $C>0$ and $B \in I^{0}\left(Z_{Q}, \Delta_{\mathscr{V}}\right.$; Hom $)$, selfadjoint with respect to $\nu$ such that

$$
A^{*} A=-B^{2}+C+R, \quad R \in I^{-\infty}\left(Z_{Q}, \Delta_{\mathscr{V}} ; \text { Hom }\right),
$$

where $A^{*}$ is taken with respect to $\nu$.

Proof. First a little explanation is in order. Note that (see [M1])

$$
I^{0}\left(Z_{Q}, \Delta_{\mathscr{V}} ; \text { Hom }\right) \subset C^{-\infty}\left(Z_{Q}, \text { Hom }\right)=C^{-\infty}(Z, \text { Hom }) .
$$

Thus every $G \in I^{0}\left(Z_{Q}, \Delta_{\mathscr{V}} ;\right.$ Hom $)$ defines

$$
\begin{array}{cccc}
G: & C^{\infty}(X, F) & \longrightarrow & C^{\infty}(X, F) \\
& C^{\infty}(\{x\} \times M, F) & \longrightarrow & C^{\infty}(\{x\} \times M, F) .
\end{array}
$$

The element $\nu$ gives a positive density on every $\{x\} \times M$, with respect to which we can define

$$
G^{*}: C^{\infty}(\{x\} \times M, F) \longrightarrow C^{\infty}(\{x\} \times M, F) .
$$

One checks that $G^{*}$ is still in $I^{0}\left(Z_{Q}, \Delta_{\mathscr{V}}\right.$; Hom $)$ and its symbol behaves like the usual symbol. (This is nothing but simultaneously taking adjoints of the operators for each parameter.) Hence (2.2) follows from the formal properties of the symbolic calculus, as in the proof of Hörmander's lemma; cf. [H, M1].

Now, using Lemma 2.3, we can verify the $L^{2}$-boundedness of $x I_{0}^{-1}\left(Z_{Q}, \Delta_{\mathscr{V}} ;\right.$ Hom $)$. Take $\chi \in C^{\infty}\left(Z_{Q}\right)$ such that $\chi \equiv 1$ on the support of the given element $A$ of $x I_{0}^{-1}$ and $\chi$ vanishes on the nonfront face of the boundary. Then by $(2.2)$

$$
\begin{aligned}
\|A \phi\|^{2} & =\left(\phi, \chi A^{*} A \chi \phi\right) \\
& =-\|\chi B \phi\|^{2}+C\|\chi \phi\|^{2}+(\phi, \chi R \phi) \\
& \leq\left(C+C^{\prime}\right)\|\phi\|^{2}, \quad \text { if }\|\chi R \phi\| \leq C^{\prime}\|\phi\| .
\end{aligned}
$$


This reduces the $L^{2}$-boundedness of $x I_{0}^{-1}$ to that of $x I_{0}^{-\infty}$, which can be handled by our previous argument.

(B) We now turn to the proof of smooth dependence of the resolvents on $x$. By the principle of uniform boundedness, it suffices to show that for $\phi, \psi \in$ $L^{2}(M, F), G \in x \mathscr{G}$, we have $F(x)=\langle G \phi, \psi\rangle \in C^{\infty}([0,+\infty))$. Here the inner product $\langle$,$\rangle on L^{2}(M, F)$ is induced by $g_{M}$. First let us note that $F(x) \in L_{\mathrm{loc}}([0,+\infty))$. In fact, one has

$$
\int|F(x)||\chi(x)| d x \leq C(\chi)\|\phi\|_{2}\|\psi\|_{2},
$$

where $C(\chi)$ is a constant depending only on $\chi$, a compactly supported smooth function on $[0, \infty)$. To see this, write out

$$
F(x)=\int G\left(x, y, z, y^{\prime}, z^{\prime}\right) \phi\left(y^{\prime}, z^{\prime}\right) \psi(y, z) d g_{x}^{\prime} d g .
$$

Then

$$
\int|F(x)||\chi(x)| d x \leq \int|\chi||G||\phi||\psi| d x d g_{x}^{\prime} d g .
$$

Since

$$
\begin{aligned}
G \in x \mathscr{G}= & x I_{0}^{-1}\left(Z_{Q}, \Delta_{\mathscr{V}} ; \text { Hom }\right)+x \rho^{p-1} C^{\infty}\left(Z_{Q}, \text { Hom }\right) \\
& +x^{p} \log R \cdot C^{\infty}(Z, \text { Hom })+x^{p} C^{\infty}(Z, \text { Hom }),
\end{aligned}
$$

one finds

$$
\begin{aligned}
\rho^{-p} G \in & x \rho^{-p} I_{0}^{-1}\left(Z_{Q}, \Delta_{\mathscr{V}} ; \text { Hom }\right)+R C^{\infty}\left(Z_{Q}, \text { Hom }\right) \\
& +R^{p} \log R \cdot C^{\infty}\left(Z_{Q}, \text { Hom }\right)+R^{p} C^{\infty}(Z, \text { Hom }) .
\end{aligned}
$$

By using Lemma 2.3, we can assume that

$$
\rho^{-p} G \in R C^{\infty}\left(Z_{Q}, \text { Hom }\right)+R^{p} \log R C^{\infty}\left(Z_{Q}, \text { Hom }\right)+R^{p} C^{\infty}(Z, \text { Hom }) .
$$

Hence $\rho^{-p} G=R^{1 / 2} G^{\prime}$ where $G^{\prime}$ is bounded continuous on $Z$.

Without loss of generality, we assume that $\phi\left(y^{\prime}, z^{\prime}\right)$ and $\psi(y, z)$ are compactly supported. Now

$$
\int|\chi||G||\phi||\psi| d x d g_{x}^{\prime} d g \leq C \int R^{-p+1 / 2}|\phi||\psi| d x d y^{\prime} d z^{\prime} d y d z .
$$

Here the integration in $x$ is over a bounded interval. Note that

$$
R=\left(x^{2}+\left|y-y^{\prime}\right|^{2}\right)^{1 / 2} \text {. }
$$

Let $R^{\prime}=\left(\left(x-x^{\prime}\right)^{2}+\left|y-y^{\prime}\right|^{2}\right)^{1 / 2}$. Then

$$
\int R^{-p+1 / 2}|\phi||\psi| d x d y^{\prime} d z^{\prime} d y d z \leq \int R^{-p+1 / 2}|\phi||\psi| d x d x^{\prime} d y^{\prime} d z^{\prime} d y d z .
$$

Here the integrations in both $x$ and $x^{\prime}$ are over bounded intervals. The desired estimate now follows from Schur's lemma. 
We now show the tangential regularity.

Lemma 2.4. $F(x)$ is conormal to $\{x=0\}: F(x) \in \mathscr{A}([0,+\infty))$.

Proof. To show this, we have to check that $\left(x \partial_{x}\right)^{k} F(x) \in L_{\mathrm{loc}}([0,+\infty))$ for any integer $k$. For $k=0$, this is (2.3). For $k=1$, note that $x \partial_{x}$ lifts trivially to $\mathscr{V}_{b}\left(Z_{Q}\right)$, tangent to $\Delta_{\mathscr{V}}$. Since $\mathscr{G}$ (thus $x \mathscr{G}$ ) is invariant under the action of this type of vector fields of $\mathscr{V}_{b}\left(Z_{Q}\right),\left(x \partial_{x}\right) G$ is also in $x \mathscr{G}$. Hence

$$
\left(x \partial_{x}\right) F(x)=\left\langle\left(x \partial_{x}\right) G \varphi, \psi\right\rangle \in L_{\text {loc }}([0,+\infty)) .
$$

Repeating this argument finishes the proof.

The next lemma gives the normal regularity.

Lemma 2.5. The Mellin transform of $F(x)$,

$$
\frac{x^{t-1}}{\Gamma(t)} F(x) \in \dot{C}^{-\infty}([0,+\infty)),
$$

extends to an entire function in $t \in C$, i.e. $F(x) \in \mathscr{A}^{\prime}([0,+\infty))$.

Proof. Note that $\frac{x^{t-1}}{\Gamma(t)} F(x)=\left\langle\left(\frac{x^{t-1}}{\Gamma(t)} G\right) \phi, \psi\right\rangle$. Hence $\frac{x^{t-1}}{\Gamma(t)} F(x)$ is holomorphic on the halfplane $\operatorname{Re} t \geq 1$. Now if $\phi \in C^{\infty}(M, F)$, then Proposition 2.1 implies $G \phi \in C^{\infty}(Z, F)$. Consequently $\frac{x^{t-1}}{\Gamma(t)} F(x)$ is entire.

For $\phi \in L^{2}(M, F)$, choose $\phi_{\delta} \in C^{\infty}(M, F)$ such that $\left\|\phi_{\delta}-\phi\right\|_{2} \rightarrow 0$ as $\delta \rightarrow 0$ and $\left\|\phi_{\delta}\right\|_{2} \leq 2\|\phi\|$. Set

$$
F_{\delta}(x)=\left\langle G \phi_{\delta}, \psi\right\rangle .
$$

We claim that $\left\{\frac{x^{t-1}}{\Gamma(t)} F_{\delta}(x)\right\}_{\delta}$ is a normal family of entire functions. Granted this, the limit of the normal family $\left\{\frac{x^{t-1}}{\Gamma(t)} F_{\delta}(x)\right\}_{\delta}$ (which exists by the normality and the principle of uniform boundedness, i.e. the Banach-Steinhaus Theorem) is also an entire function with values in $\dot{C}^{-\infty}([0,+\infty))$. On the other hand, one has for $\operatorname{Re} t>1, \frac{x^{t-1}}{\Gamma(t)} F_{\delta}(x) \rightarrow \frac{x^{t-1}}{\Gamma(t)} F(x)$ weakly by $(2.3)$. This proves the lemma.

It remains to establish the claim. We show that the family is locally uniformly bounded. For this note that for $\operatorname{Re} t>1$ and $\chi \in C^{\infty}([0, \infty))$ compactly supported,

$$
\begin{aligned}
\left|\int_{0}^{\infty} \frac{x^{t-1}}{\Gamma(t)} F_{\delta}(x) \chi(x) d x\right| & \leq \frac{C(\chi) a(\chi)^{\operatorname{Re} t-1}}{|\Gamma(t)|} \int\left|F_{\delta}\right||\chi| d x \\
& \leq \frac{C(\chi) a(\chi)^{\operatorname{Re} t-1}}{|\Gamma(t)|}\|\phi\|_{2}\|\psi\|_{2},
\end{aligned}
$$

where the last inequality comes from $(2.3)$ (and $\left.\left\|\phi_{\delta}\right\|_{2} \leq 2\|\phi\|\right)$. Since $\frac{x^{t-1}}{\Gamma(t)} \stackrel{\text { def }}{=}$ $\gamma(t)$ satisfies the functional equation

$$
\frac{d}{d x} \gamma(t)=\gamma(t-1),
$$


using integration by parts, we have for $\operatorname{Re} t>-k+1$,

$$
\left|\int_{0}^{\infty} \frac{x^{t-1}}{\Gamma(t)} F_{\delta}(x) \chi(x) d x\right| \leq \frac{C(\chi) a(\chi)^{\operatorname{Re} t+k-1}}{|\Gamma(t+k)|} \sum_{i=0}^{k}\left\|\partial_{x}^{i} F_{\delta}\right\|_{L_{\mathrm{loc}}^{1}} .
$$

We want to estimate $\left\|\partial_{x}^{k} F_{\delta}\right\|_{L_{\text {loc }}^{1}}$ in terms of $\|\phi\|_{2}$ and $\|\psi\|_{2}$. Without loss of generality let us assume that the $F$ 's and $F_{\delta}$ 's have compact support. Define

$$
F_{M}(t)=\int_{0}^{\infty} x^{t-1} F(x) d x, \quad F_{\gamma}(t)=\int_{0}^{\infty} \frac{x^{t-1}}{\Gamma(t)} F(x) d x
$$

Then

$$
F_{\gamma}(t)=\frac{F_{M}(t)}{\Gamma(t)}
$$

and

(a) $\left(\partial_{x} F\right)_{M}(t)=-(t-1) F_{M}(t-1)$.

(b) $\left(x \partial_{x} F\right)_{M}(t)=-t F_{M}(t)$.

(c) (Plancherel's identity) $\left\langle F, \overline{F^{\prime}}\right\rangle=\int_{\operatorname{Re} t=0} F_{M}(t) \bar{F}_{M}^{\prime}(\bar{t}) d t$.

The verifications of (a) and (b) are just computations, while (c) follows from Plancherel's identity for the Fourier transform, when we make the substitution $x=e^{-i u}$.

Now $F(x) \in C^{\infty}([0,+\infty))$ implies that $F_{\gamma}(t)$ is entire. It follows from (2.6) that $F_{M}(t)$ is then meromorphic with at most simple poles at negative integers. If, in addition, $F(x)$ vanishes near $x=0, F_{M}(t)$ is then entire. This can be seen trivially from the definition.

Take $\varphi(x) \in C^{\infty}([0,+\infty))$ identically 1 on $[1,+\infty)$ and 0 on $[0,1 / 2]$, and put $\varphi_{\varepsilon}(x)=\varphi(x / \varepsilon)$. Apply Plancherel's identity to $\varphi_{\varepsilon}(x) \partial_{x}^{k} F_{\delta}(x)$ to obtain

$$
\int_{0}^{\infty} \varphi_{\varepsilon}^{2}(x)\left|\partial_{x}^{k} F_{\delta}(x)\right|^{2} d x=\int_{\operatorname{Re} t=0}\left(\varphi_{\varepsilon} \partial_{x}^{k} F_{\delta}\right)_{M}(t) \overline{\left(\varphi_{\varepsilon} \partial_{x}^{k} F_{\delta}\right)_{M}(\bar{t})} d t
$$

By the above discussion, $\left(\varphi_{\varepsilon} \partial_{x}^{k} F_{\delta}\right)_{M}(t) \overline{\left(\varphi_{\varepsilon} \partial_{x}^{k} F_{\delta}\right)_{M}(\bar{t})}$ is entire, which allows us to shift the line of integration $\operatorname{Re} t=0$ to any other such line. Hence

$$
\int_{0}^{\infty} \varphi_{\varepsilon}^{2}(x)\left|\partial_{x}^{k} F_{\delta}(x)\right|^{2} d x=\int_{\operatorname{Re} t=k+3}\left(\varphi_{\varepsilon} \partial_{x}^{k} F_{\delta}\right)_{M}(t) \overline{\left(\varphi_{\varepsilon} \partial_{x}^{k} F_{\delta}\right)_{M}(\bar{t})} d t .
$$


Letting $\varepsilon \rightarrow 0$ and noting that for $\operatorname{Re} t>0,\left(\varphi_{\varepsilon} \partial_{x}^{k} F_{\delta}\right)_{M}(t) \rightarrow\left(\partial_{x}^{k} F_{\delta}\right)_{M}(t)$, we deduce

$$
\begin{aligned}
\int_{0}^{\infty} & \left|\partial_{x}^{k} F_{\delta}(x)\right|^{2} d x \\
\quad & =\int_{\operatorname{Re} t=k+3}\left(\partial_{x}^{k} F_{\delta}\right)_{M}(t) \overline{\left(\partial_{x}^{k} F_{\delta}\right)_{M}(\bar{t})} d t \\
& =\int_{\operatorname{Re} t=k+3}(t-1)^{2} \cdots(t-k-1)^{2}\left(F_{\delta}\right)_{M}(t-k-1) \overline{\left(F_{\delta}\right)_{M}(\bar{s}-k-1)} d t
\end{aligned}
$$

To estimate $\left(F_{\delta}\right)_{M}(t)$, we use (b) above. Thus for any large $N$,

$$
\left|\left(F_{\delta}\right)_{M}(t)\right| \leq C(1+|t|)^{-N} \sum_{j=0}^{N}\left|\left(\left(x \partial_{x}\right)^{j} F_{\delta}\right)_{M}(t)\right| .
$$

By the invariance of $x \mathscr{G}$ under the action of $x \partial_{x},\left(x \partial_{x}\right)^{j} F_{\delta}$ obeys an estimate of the type (2.3), which yields

$$
\left|\left(\left(x \partial_{x}\right)^{j} F_{\delta}\right)_{M}(t)\right| \leq C a^{\operatorname{Re} t-1}\|\phi\|_{2}\|\psi\|_{2},
$$

provided $\operatorname{Re} t>1$. Combining these two, we finally arrive at

$$
\left|\left(F_{\delta}\right)_{M}(t)\right| \leq C a^{\operatorname{Re} t-1}(1+|t|)^{-N}\|\phi\|_{2}\|\psi\|_{2} .
$$

The claim follows from (2.5), (2.7), and (2.8) by taking $N>k+1$.

Now the smooth dependence of the resolvents on the parameter follows from Lemmas 2.4, 2.5 and the following fact (see [M2]),

$$
\mathscr{A}([0,+\infty)) \cap \mathscr{A}^{\prime}([0,+\infty))=C^{\infty}([0,+\infty))
$$

2.2. Asymptotic behavior of spectrum. The proceeding analysis of the resolvent of $\frac{1}{x} D_{x}$ bears important implications for the spectrum of $D_{x}$ in the adiabatic limit. However, before one can apply regular perturbation theory to obtain the asymptotic behavior of $\operatorname{spec}\left(D_{x}\right)$, one needs certain finiteness results. To this end we now take a closer look at our operator by exploring the local geometry of the fibration.

In terms of the decomposition

$$
L^{2}(M, F)=\text { Image } P^{\perp} \oplus \text { Image } P,
$$

where $P=P_{\operatorname{ker} D_{Y}}$, our operator can be written in the matrix form

$$
\frac{1}{x} D_{x}=\left(\begin{array}{ll}
A_{1} & A_{2} \\
A_{2}^{*} & A_{3}
\end{array}\right)
$$


where (see (1.3))

$$
\begin{aligned}
& A_{1}=x^{-1} D_{Y}+P^{\perp} \widetilde{D}_{B} P^{\perp}+x P^{\perp} \frac{T}{4} P^{\perp}, \\
& A_{2}=P^{\perp} \widetilde{D}_{B} P+x P^{\perp} \frac{T}{4} P, \\
& A_{3}=D_{0}+x P \frac{T}{4} P .
\end{aligned}
$$

Lemma 2.6. (a) There exists a constant $\bar{\lambda}_{0}>0$ such that $\operatorname{spec}\left(\left|A_{1}\right|\right)$ is contained in the halfline $\left[\bar{\lambda}_{0} / x,+\infty\right)$.

(b) $A_{2}$ (consequently $A_{2}^{*}$ ) is bounded on $L^{2}(M, F)$.

Proof. Statement (a) is essentially Proposition 4.41 in [BC2].

(b) First note that the commutator $\left[D_{Y}, \widetilde{D}_{B}\right] \stackrel{\text { def }}{=} D_{Y} \widetilde{D}_{B}+\widetilde{D}_{B} D_{Y}$ is a first order differential operator, which acts fiberwise (cf. [BC2, $\S 4(a)(b)])$. On the other hand, $P^{\perp}\left[D_{Y}, \widetilde{D}_{B}\right] P=P^{\perp} D_{Y} \widetilde{D}_{B} P$ is fiberwisely of finite rank. Therefore $P^{\perp} D_{Y} \widetilde{D}_{B} P=\left(P^{\perp} D_{Y} P^{\perp}\right) P^{\perp} \widetilde{D}_{B} P$ is bounded on $L^{2}(M, F)$. But $\left(P^{\perp} D_{Y} P^{\perp}\right)^{-1}$ is also bounded on $L^{2}(M, F)$. It follows that $P^{\perp} \widetilde{D}_{B} P$, consequently $A_{2}=$ $P^{\perp} \widetilde{D}_{B} P+x P^{\perp} \frac{T}{4} P$, is bounded.

To have a first picture of $\operatorname{spec}\left(D_{x}\right)$, we deform $\frac{1}{x} D_{x}$ to an operator whose spectrum we know much more about. For this purpose, the following lemma is quite useful.

Lemma 2.7. If $T(\varepsilon)$ is an analytic family of selfadjoint operators on a Hilbert space. Then its eigenvalue $\lambda(\varepsilon)$ (which can be arranged to be analytic in $\varepsilon$ ) satisfies

$$
\lambda^{\prime}(\varepsilon)=\left(T^{\prime}(\varepsilon) \phi(\varepsilon), \phi(\varepsilon)\right),
$$

where $\phi(\varepsilon)$ is a normalized eigenvector associated with the eigenvalue $\lambda(\varepsilon)$. In particular,

$$
\left|\lambda^{\prime}(\varepsilon)\right| \leq\left\|T^{\prime}(\varepsilon)\right\| .
$$

For the proof, see [K, p. 391].

Lemma 2.8. Consider the operator

$$
T=\left(\begin{array}{cc}
A_{1} & 0 \\
0 & A_{3}
\end{array}\right) \text {. }
$$

Its eigenvalues $\bar{\lambda}_{x}$ are analytic in $x$ for $x>0$. Furthermore for the positive constant $\bar{\lambda}_{0}$ of Lemma 2.6, either

$$
\left|\bar{\lambda}_{x}\right| \geq \bar{\lambda}_{0} / x
$$

or $\bar{\lambda}_{x}$ is analytic at $x=0$,

$$
\bar{\lambda}_{x}=\lambda_{0}^{\prime}+\lambda_{1}^{\prime} x+\cdots, \quad \text { with } \lambda_{0}^{\prime} \in \operatorname{spec}\left(D_{0}\right) .
$$


Moreover, for each $\bar{\lambda}_{x}$ there corresponds an eigenvalue $\frac{1}{x} \lambda_{x}$ of $\frac{1}{x} D_{x}$ so that

$$
\left|\frac{1}{x} \lambda_{x}-\bar{\lambda}_{x}\right| \leq C
$$

for a uniform constant $C$.

Proof. The eigenvalues of $T$ are either eigenvalues of $A_{1}$ or that of $A_{3}$. Accordingly, we call them of type I or II. By the previous lemma, the eigenvalues $\bar{\lambda}_{x}$ of type I, i.e. of $A_{1}$ satisfy

$$
\left|\bar{\lambda}_{x}\right| \geq \bar{\lambda}_{0} / x
$$

On the other hand $A_{3}=D_{0}+x P \frac{T}{4} P$ is an entire family of selfadjoint operators (in the sense of Kato). Therefore its eigenvalues $\bar{\lambda}_{x}$ (type II) depend analytically on $x$ and we have (2.11). In this case, we also have a uniform remainder estimate

$$
\bar{\lambda}_{x}=\lambda_{0}^{\prime}+x C(x)
$$

with $|C(x)| \leq C$ uniformly. This can be shown by the argument below, using the deformation $A_{3}(\lambda)=D_{0}+\lambda x P \frac{T}{4} P$ and the fact that $\left\|P \frac{T}{4} P\right\| \leq C$.

Now consider the deformation

$$
T(\lambda)=\left(\begin{array}{cc}
A_{1} & \lambda A_{2} \\
\lambda A_{2}^{*} & A_{3}
\end{array}\right),
$$

we have $T(0)=T$ and $T(1)=\frac{1}{x} D_{x}$. For each fixed $x>0, T(\lambda)$ is an entire family of selfadjoint operators. Therefore its eigenvalues depend analytically on $\lambda$. Thus for each $\bar{\lambda}_{x}$ of $T(0)$, there corresponds a $\frac{1}{x} \lambda_{x}$ of $T(1)$. Moreover (see Lemma 2.7)

$$
\left|\frac{1}{x} \lambda_{x}-\bar{\lambda}_{x}\right| \leq \int_{0}^{1}\left\|T^{\prime}(\lambda)\right\| d \lambda \leq C,
$$

where the last inequality follows from (b) of Lemma 2.6.

We are now ready to prove Theorem 1.5.

Proof of Theorem 1.5. For $x>0, D_{x}$ is what is called in [K] the analytic family of type (B) of selfadjoint operators. The first statement follows immediately. Thus the main point here is the study of the behavior of $\lambda_{x}$ as $x \rightarrow 0$.

Lemma 2.8 already contains important information about $\operatorname{spec}\left(D_{x}\right)$. In fact if $\frac{1}{x} \lambda_{x}$ corresponds to $\bar{\lambda}_{x}$, which is of type I, then $x\left|\bar{\lambda}_{x}\right| \geq \bar{\lambda}_{0}$ and (2.14) implies that

$$
\left|\lambda_{x}\right| \geq x\left|\bar{\lambda}_{x}\right|-C x \geq \bar{\lambda}_{0} / 2 \stackrel{\text { def }}{=} \lambda_{0}
$$

provided $x \leq \bar{\lambda}_{0} / 2 C$. On the other hand, if $\bar{\lambda}_{x}$ is of type II, we have

$$
\left|\frac{1}{x} \lambda_{x}\right| \leq\left|\bar{\lambda}_{x}\right|+C \leq \lambda_{0}^{\prime}+2 C
$$

by (2.13). In particular $\left\{\frac{1}{x} \lambda_{x}\right\}$ are uniformly bounded. They must converge as $x \rightarrow 0$ to an eigenvalue $\lambda_{1}$ of $D_{0}$. Otherwise, a subsequence of $\left\{\frac{1}{x} \lambda_{x}\right\}$ 
converges to $\lambda^{\prime} \notin \operatorname{spec}\left(D_{0}\right)$. This implies that the resolvent $\left(\frac{1}{x} D_{x}-\lambda^{\prime}\right)^{-1}$ cannot exist no matter how small $x$ is, in contradiction to Proposition 2.1.

Suppose $\varepsilon_{0}>0$ is chosen such that $\lambda_{1} \pm \varepsilon_{0} \notin \operatorname{spec}\left(D_{0}\right)$ and $\frac{1}{x} \lambda_{x}$ 's are in the $\varepsilon_{0}$-ball around $\lambda_{1}$ for $x$ small. By Theorem 2.2,

$$
P(x)=\frac{1}{2 \pi i} \int_{\left|\lambda-\lambda_{1}\right|=\varepsilon_{0}}\left(\frac{1}{x} D_{x}-\lambda\right)^{-1} d \lambda
$$

is well defined and $C^{\infty}$ in $x$ as a family of orthogonal projections. The following lemma says that they are unitarily equivalent to each other.

Lemma 2.9. Let $P$ and $Q$ be (orthogonal) projections on a Hilbert space with $\|P-Q\|<1$. Then

$$
W=\left[1-(P-Q)^{2}\right]^{-1 / 2}[P Q+(1-P)(1-Q)]
$$

is well defined and invertible (unitary). Furthermore,

$$
\begin{gathered}
W^{-1}=[(1-Q)(1-P)+Q P]\left[1-(P-Q)^{2}\right]^{-1 / 2}, \\
W^{-1} P W=Q .
\end{gathered}
$$

For the proof of this lemma see [RS, p. 72].

Lemma 2.9 applied to the family $P(x)$ produces a smooth family of unitary operators $U(x)$ such that

$$
U(x)^{-1} P(x) U(x)=P(0) \quad(x \text { small }) .
$$

Moreover, $U(x)^{-1}\left(\frac{1}{x} D_{x}-\lambda_{1}-\varepsilon_{0}\right)^{-1} U(x)$ is a $C^{\infty}$ family on Image $P(0)$ :

$$
\begin{array}{ccc}
\text { Image } P(x) & \stackrel{\left(\frac{1}{x} D_{x}-\lambda_{1}-\varepsilon_{0}\right)^{-1}}{\longrightarrow} & \text { Image } P(x) \\
\uparrow U(x) & & \uparrow U(x) \\
\text { Image } P(0) & \longrightarrow & \text { Image } P(0) .
\end{array}
$$

We claim that $\operatorname{dim}(\operatorname{Image} P(0))<+\infty$. In fact, being a smooth family of projections,

$$
\operatorname{rank} P(x)=\operatorname{rank} P(0) \text {. }
$$

Further, by the discreteness of $\operatorname{spec}\left(D_{x}\right)$ and functional analysis, for $x>0$,

$$
P(x)=\bigoplus_{\left|\frac{1}{x} \lambda_{x}-\lambda_{1}\right|<\varepsilon_{0}} P_{\lambda_{x}}
$$

where $P_{\lambda_{x}}$ is the orthogonal projection onto the $\lambda_{x}$-eigenspace. In other words, rank $P(x)$ equals to the number of $\lambda_{x}$ (counted with multiplicity) such that $\frac{1}{x} \lambda_{x}$ lies in an $\varepsilon_{0}$-ball around $\lambda_{1}$. For $x$ small, such $\lambda_{x}$ could only correspond 
to the eigenvalues $\bar{\lambda}_{x}$ of $A_{3}$, which obey

$$
\bar{\lambda}_{x}=\lambda_{0}^{\prime}+\lambda_{1}^{\prime} x+\cdots
$$

with $\lambda_{0}^{\prime} \in \operatorname{spec}\left(D_{0}\right)$. This, together with (2.14) and (2.13), implies rank $P(x)$ is bounded from above by the number of eigenvalues of $D_{0}$ lying in a $\left(2 C+\varepsilon_{0}\right)$ ball around $\lambda_{1}$, counted with multiplicity. There are only finitely many of them, yielding

$$
\text { rank } P(x)<+\infty \text {, }
$$

i.e.

$$
\operatorname{dim}(\text { Image } P(0))<+\infty \text {. }
$$

Thus $U(x)^{-1}\left(\frac{1}{x} D_{x}-\lambda_{1}-\varepsilon_{0}\right)^{-1} U(x)$ is a smooth family on a finite-dimensional vector space. By standard functional analysis so is its inverse $U(x)^{-1}\left(\frac{1}{x} D_{x}-\lambda_{1}-\varepsilon_{0}\right) U(x)=U(x)^{-1}\left(\frac{1}{x} D_{x}\right) U(x)-\lambda_{1}-\varepsilon_{0}$. One can then apply the following lemma (cf. [Sn, Lemma 5.2]), showing the existence of complete asymptotic expainsions for those $\lambda_{x}$ with $\frac{1}{x} \lambda_{x}$ lying near an eigenvalue of $D_{0}$.

Lemma 2.10. Let $C(x)$ be a family of symmetric matrices whose elements have complete asymptotic expansion in $x$ as $x \rightarrow 0$. Then the eigenvalues of $C(x)$ have complete asymptotic expansions.

It remains to prove $(1.11)$. Choose $\lambda \notin \operatorname{spec}\left(D_{0}\right)$ real and consider

$$
S(\varepsilon)=\left(\begin{array}{cc}
A_{1}-\lambda & \varepsilon A_{2} \\
\varepsilon A_{2}^{*} & A_{3}-\lambda
\end{array}\right) .
$$

Set $T(\varepsilon)=S(\varepsilon)^{-1}$. One has

$$
\frac{d}{d \varepsilon} T(\varepsilon)=S(\varepsilon)^{-1} \frac{d S(\varepsilon)}{d \varepsilon} S(\varepsilon)^{-1}=T(\varepsilon)\left(\begin{array}{cc}
0 & A_{2} \\
A_{2}^{*} & 0
\end{array}\right) T(\varepsilon) .
$$

By straightforward calculation, one finds

$$
T(\varepsilon)=\left(\begin{array}{ll}
T_{1} & T_{2} \\
T_{2}^{*} & T_{3}
\end{array}\right)
$$

where

$$
\begin{aligned}
& T_{1}=\left(A_{1}-\lambda-\varepsilon^{2} A_{2}\left(A_{3}-\lambda\right)^{-1} A_{2}^{*}\right)^{-1}, \\
& T_{2}=-\varepsilon\left(A_{1}-\lambda\right)^{-1} A_{2}\left(A_{3}-\lambda-\varepsilon^{2} A_{2}^{*}\left(A_{1}-\lambda\right)^{-1} A_{2}\right)^{-1}, \\
& T_{3}=\left(A_{3}-\lambda-\varepsilon^{2} A_{2}^{*}\left(A_{1}-\lambda\right)^{-1} A_{2}\right)^{-1} .
\end{aligned}
$$

From Lemma 2.6, we have

$$
\left\|T_{1}\right\| \leq C x, \quad\left\|T_{2}\right\| \leq C x, \quad\left\|T_{3}\right\| \leq C .
$$

It follows then

$$
\left\|\frac{d}{d \varepsilon} T(\varepsilon)\right\| \leq C x
$$


This, together with (2.9), implies $\left|\lambda^{\prime}(\varepsilon)\right| \leq C x$. But $\lambda(1)=\left(\frac{\lambda_{x}}{x}-\lambda\right)^{-1}$ and $\lambda(0)=\left(\bar{\lambda}_{x}-\lambda\right)^{-1}$ where $\bar{\lambda}_{x}$ is the same as in Lemma 2.8. Thus

$$
\left|\left(\lambda_{x} / x-\lambda\right)^{-1}-\left(\bar{\lambda}_{x}-\lambda\right)^{-1}\right| \leq C x,
$$

or

$$
\left|\lambda_{x} / x-\bar{\lambda}_{x}\right| \leq C x\left|\lambda_{x} / x-\lambda\right|\left|\bar{\lambda}_{x}-\lambda\right|
$$

Since $\lambda_{x} / x \rightarrow \lambda_{1}$, we must have $\bar{\lambda}_{x}=\lambda_{1}+C(x) x$, where $|C(x)| \leq C$. Plugging in (2.16), and using the estimate (2.12) of Lemma 2.8, one obtains

$$
\left|\lambda_{x} / x-\lambda_{1}\right| \leq C x \lambda_{1}^{2} \text {. }
$$

This is $(1.11)$.

\section{THE UNIFORM ASYMPTOTIC EXPANSION}

In what follows we also use the notation $D_{x} e^{-t D_{x}^{2}}$ to denote its own kernel taken with respect to the metric $g_{M}$. Therefore $\operatorname{tr}\left(D_{x} e^{-t D_{x}^{2}}\right)$ could mean either the integrated trace or the pointwise trace, depending on the situation. We make it clear in the context.

Theorem 3.1. Pointwisely, one has the following uniform asymptotic expansion (compare [BC2, (4.81)]),

$$
\operatorname{tr}\left(D_{x} e^{-t D_{x}^{2}}\right)=\sum_{i=-n}^{N-1} a_{i}(t)\left(t x^{2}\right)^{i / 2}+O\left(\left(t x^{2}\right)^{N / 2}\right), \quad \text { as } x \rightarrow 0,
$$

where the $a_{i}(t)$ 's are bounded for $t \geq 1$, and so is $O(\cdot)$.

This section is devoted to the proof of this statement. We first localize the problem, i.e., we transplant the problem to a trivial fibration whose base space is an Euclidean space. Then we construct a parametrix by taking the product of the heat kernels on the base and along the fiber, and then applying Duhamel's principle. The asymptotic expansion comes from the standard heat expansion.

Remark. In [DM], as indicated, we examine the heat kernel of $D_{x}^{2}$ on an appropriate blow-up space. As a consequence, we obtain a more general expansion.

3.1. Localization. Fix a point $y \in B$, let $U=B_{r_{0}}(y)$ in the metric $g_{B}$ for a fixed small number $r_{0} \geq 0$. Denote by $K_{x}\left(t, y, z, y^{\prime}, z^{\prime}\right)$ the kernel of $D_{x} e^{-t D_{x}}$ with respect to the metric $g=\pi^{*} g_{B}+g_{Y}$.

Lemma 3.2. $K_{x}\left(t, y, z, y^{\prime}, z^{\prime}\right)$ decays exponentially as $x \rightarrow 0$ for those $y^{\prime} \notin$ $U$, i.e., there exist positive constants $C$ and $C^{\prime}$ such that

$$
\left|K_{x}\left(t, y, z, y^{\prime}, z^{\prime}\right)\right| \leq C x^{-p} e^{-C^{\prime}{\overline{y, y^{2}}}^{2} / t x^{2}} .
$$

One has the same bounds for the derivatives in $y, z, y^{\prime}, z^{\prime}$. 
Proof. This follows essentially from the theorem of Cheeger-Gromov-Taylor. Let $\widetilde{K}_{x}\left(t, y, z, y^{\prime}, z^{\prime}\right)$ be the kernel of $D_{x} e^{-t D_{x}}$ with respect to the metric $g_{x}$, then

$$
K_{x}\left(t, y, z, y^{\prime}, z^{\prime}\right)=x^{-p} \widetilde{K}_{x}\left(t, y, z, y^{\prime}, z^{\prime}\right) .
$$

By estimate (1.30) in [CGT],

$$
\left|\widetilde{K}_{x}\left(t, y, z, y^{\prime}, z^{\prime}\right)\right| \leq \frac{C(n)}{\pi^{2}} \sum_{0 \leq i, j \leq N} \int_{d_{x}-r}^{\infty}\left|\hat{f}^{(2 i+2 j)}(s)\right| d s,
$$

where $r=\min \left(r_{0} / 3, \operatorname{inj}(M, g)\right), f(\lambda)=\lambda e^{-t \lambda^{2}} \stackrel{\text { def }}{=} \lambda g(\lambda)$, and $d_{\underline{x}}=$ the distance in $g_{x}$ between $(y, z)$ and $\left(y^{\prime}, z^{\prime}\right)$. Clearly $d_{x} \geq \overline{y, y^{\prime}} / x \geq \overline{y, y^{\prime}} / 2 x+r$ for $x$ small. Note that (see [CGT, p. 28])

$$
\left|\hat{f}^{(k)}(s)\right|=\left|\hat{g}^{(k+1)}(s)\right| \leq \frac{C(k)}{t^{(k+1) / 2}} t^{-1 / 2} e^{-s^{2} / 8 t} .
$$

Therefore,

$$
\begin{aligned}
\left|\widetilde{K}_{x}\left(t, y, z, y^{\prime}, z^{\prime}\right)\right| & \leq \frac{C}{\sqrt{t}} \int_{\frac{y, y^{\prime}}{+\infty} / 2 x} t^{-1 / 2} e^{-s^{2} / 8 t} d t \\
& \leq \frac{C}{\sqrt{t}} e^{-\overline{y, y^{\prime}} / 32 t x^{2}} .
\end{aligned}
$$

This implies (3.2). The estimates for the derivatives are similar.

Let $r_{0}$ be small so that $U$ is a trivializing neighborhood of $\pi$. Consider now the trivial fibration

$$
\begin{gathered}
Y \longrightarrow \bar{M}=Y \times \mathbb{R}^{p} \longrightarrow \mathbb{R}^{p}, \\
\bar{g}=\bar{\pi}^{*} g_{\mathbb{R}^{p}}+g_{Y},
\end{gathered}
$$

where $g_{\mathbb{R}^{p}}$ coincides with $g_{B}$ in a neighborhood of 0 and is flat outside a compact domain. $g_{Y}$ coincides with $g_{Y, B}$ in a neighborhood of 0 and is constant outside the compact domain. Correspondingly, we have $\bar{D}_{x}$. Let $\bar{K}_{x}\left(t, y, z, y^{\prime}, z^{\prime}\right)$ be the kernel of $\bar{D}_{x} e^{-t \bar{D}_{x}^{2}}$ with respect to $\bar{g}$. The following lemma says that instead of considering $D_{x}$, we can just as well consider $\bar{D}_{x}$.

Lemma 3.3. $K_{x}$ and $\bar{K}_{x}$ differ by an exponentially decaying term,

$$
\left|K_{x}\left(t, y, z, y^{\prime}, z^{\prime}\right)-\bar{K}_{x}\left(t, y, z, y^{\prime}, z^{\prime}\right)\right| \leq C t x^{-n} e^{-C^{\prime} / t x^{2}}
$$

Proof. Basically this is because inside a small neighborhood the two operators coincide while outside the neighborhood the heat kernels decay exponentially. Let $G_{x}$ and $\bar{G}_{x}$ be the kernels of $e^{-t D_{x}}$ and $e^{-t \bar{D}_{x}}$. Then

$$
K_{x}=D_{x} G_{x}, \quad \bar{K}_{x}=\bar{D}_{x} \bar{G}_{x} .
$$


Choose a smooth function $\phi=\phi\left(y, y^{\prime}\right)$ such that $\phi\left(y, y^{\prime}\right) \equiv 1$ when $y^{\prime} \in U / 3$ and $\phi\left(y, y^{\prime}\right) \equiv 0$ when $y^{\prime} \notin U$. Consider

$$
\begin{gathered}
G_{x}\left(t, y, z, y^{\prime \prime}, z^{\prime \prime}\right) \phi\left(y, y^{\prime \prime}\right)-\bar{G}_{x}\left(t, y, z, y^{\prime \prime}, z^{\prime \prime}\right) \phi\left(y, y^{\prime \prime}\right) \\
=\int_{0}^{t} \frac{\partial}{\partial s} \int G_{x}\left(s, y, z, y^{\prime}, z^{\prime}\right) \bar{G}_{x}\left(t-s, y^{\prime}, z^{\prime}, y^{\prime \prime}, z^{\prime \prime}\right) \\
\times \phi\left(y, y^{\prime}\right) \phi\left(y^{\prime}, y^{\prime \prime}\right) d y^{\prime} d z^{\prime} d s \\
=-\int_{0}^{t} \int G_{x}\left(s, y, z, y^{\prime}, z^{\prime}\right)\left(D_{x}-\bar{D}_{x}\right) \bar{G}_{x}\left(t-s, y^{\prime}, z^{\prime}, y^{\prime \prime}, z^{\prime \prime}\right) \\
\times \phi\left(y, y^{\prime}\right) \phi\left(y^{\prime}, y^{\prime \prime}\right) d y^{\prime} d z^{\prime} d s .
\end{gathered}
$$

Since $D_{x}=\bar{D}_{x}$ in $U$, one has

$$
\begin{aligned}
& K_{x}\left(t, y, z, y^{\prime \prime}, z^{\prime \prime}\right) \phi\left(y, y^{\prime \prime}\right)-\bar{K}_{x}\left(t, y, z, y^{\prime \prime}, z^{\prime \prime}\right) \phi\left(y, y^{\prime \prime}\right) \\
&=-\int_{0}^{t} \int D_{x} G_{x}\left(s, y, z, y^{\prime}, z^{\prime}\right)\left(D_{x}-\bar{D}_{x}\right) \bar{G}_{x}\left(t-s, y^{\prime}, z^{\prime}, y^{\prime \prime}, z^{\prime \prime}\right) \\
& \times \phi\left(y, y^{\prime}\right) \phi\left(y, y^{\prime \prime}\right) d y^{\prime} d z^{\prime} d s \\
&=-\int_{0}^{t} \int_{y^{\prime}, y \geq r_{0}}\left(D_{x}-\bar{D}_{x}\right) D_{x} G_{x}\left(s, y, z, y^{\prime}, z^{\prime}\right) \bar{G}_{x}\left(t-s, y^{\prime}, z^{\prime}, y^{\prime \prime}, z^{\prime \prime}\right) \\
& \times \phi\left(y, y^{\prime}\right) \phi\left(y, y^{\prime \prime}\right) d y^{\prime} d z^{\prime} d s .
\end{aligned}
$$

Applying the previous estimates on the derivatives of $G_{x}$ and using the fact that as an $L^{2}$ operator $\bar{G}_{x}$ has norm bounded by 1 , we find

$$
\left|K_{x}\left(t, y, z, y^{\prime \prime}, z^{\prime \prime}\right) \phi\left(y, y^{\prime \prime}\right)-\bar{K}_{x}\left(t, y, z, y^{\prime \prime}, z^{\prime \prime}\right) \phi\left(y, y^{\prime \prime}\right)\right| \leq C t x^{-p} e^{-C^{\prime} / t x^{2}} \text {. }
$$

In particular, one has

$$
\left|K_{x}\left(t, y, z, y^{\prime \prime}, z^{\prime \prime}\right)-\bar{K}_{x}\left(t, y, z, y^{\prime \prime}, z^{\prime \prime}\right)\right| \leq C t x^{-n} e^{-C^{\prime} / t x^{2}}
$$

when $\overline{y, y^{\prime \prime}} \leq r_{0} / 3$. For those $y, y^{\prime \prime}$ such that $\overline{y, y^{\prime \prime}} \geq r_{0} / 3$ each of $K_{x}$ and $\bar{K}_{x}$ are exponentially decaying by (3.2). Combining the two finishes the proof.

3.2. A "rough" parametrix. By the above result we can replace the original fibration by the trivial one

$$
Y \longrightarrow \bar{M}=Y \times \mathbb{R}^{p} \longrightarrow \mathbb{R}^{p}
$$

We now construct a parametrix for our operator living on the trivial fibration. For the simplicity of notations we suppress the "bar's" below. 
Recall that the tangent bundle of $M$ splits as the Whitney sum of its vertical space and its horizontal space by the connection,

$$
T M=T^{V} \oplus T^{H}
$$

and the horizontal lift gives an isomorphism

$$
T_{y} \mathbb{R}^{p} \stackrel{\simeq}{\longrightarrow} T_{(y, z)}^{H}
$$

for any $z \in \pi^{-1}(y)$. Also we have by (1.3),

$$
D_{x}=D_{Y}+x \widetilde{D}_{B}+x^{2} T / 4,
$$

where $\widetilde{D}_{B}$ is the Dirac operator on $\mathbb{R}^{p}$ lifted via the isomorphism (3.3). Taking the square of (3.4), we have

$$
D_{x}^{2}=D_{Y}^{2}+x\left[D_{Y}, \widetilde{D}_{B}\right]+x^{2} \widetilde{D}_{B}^{2}+x^{2}\left[D_{Y}, \frac{T}{4}\right]+x^{3}\left[\widetilde{D}_{B}, \frac{T}{4}\right]+x^{4}\left(\frac{T}{4}\right)^{2} .
$$

Recall the basic fact that $\left[D_{Y}, \widetilde{D}_{B}\right]$ is a first order differential operator acting fiberwise. Thus we can construct the heat kernel $F_{y}\left(t, x, z, z^{\prime}\right)$ of $D_{Y}^{2}+$ $x\left[D_{Y}, \widetilde{D}_{B}\right]$. Here the subscript indicates its dependence on the base variable. Let $E\left(t, y, y^{\prime}\right)$ be the heat kernel of $e^{-t \widetilde{D}_{B}^{2}}$, originally living on $\mathbb{R}^{p}$, and lifted to $M$ via the horizontal lift. Consider

$$
\begin{gathered}
K_{x}\left(t, y, z, y^{\prime}, z^{\prime}\right)=F_{y}\left(t, x, z, z^{\prime}\right) E\left(t x^{2}, y, y^{\prime}\right), \\
G_{x}\left(t, y, z, y^{\prime}, z^{\prime}\right)=\left(\partial / \partial t+D_{x}^{2}\right) K_{x}\left(t, y, z, y^{\prime}, z^{\prime}\right) .
\end{gathered}
$$

Note that here and throughout, we use $y$ 's as the coordinates of the base, $\mathbb{R}^{p}$, and $z$ 's as the coordinates of the fiber $Y$. One has

Lemma 3.4. $K_{x}$ is a "rough" parametrix for $\partial / \partial t+D_{x}^{2}$, i.e. it satisfies the estimate

$$
\left|\left(\partial / \partial t+D_{x}^{2}\right) K_{x}\right| \leq C\left(t x^{2}\right)^{-n / 2-1 / 2} x^{2}
$$

for $t \geq 1$.

Proof. We calculate,

$$
\begin{aligned}
G_{x}= & \left(\partial / \partial t F_{y}\right) E+F_{y}(\partial / \partial t E)+\left(\left(D_{Y}^{2}+x\left[D_{Y}, \widetilde{D}_{B}\right]\right) F_{y} E+x^{2}\left(\widetilde{D}_{B}^{2} F_{y}\right) E\right. \\
& +x^{2}\left(\widetilde{D}_{B}^{\prime} F_{y}\right)\left(\widetilde{D}_{B}^{\prime \prime} E\right)+x^{2} F_{y}\left(\widetilde{D}_{B}^{2} E\right)+x^{2} F_{y}(R E)+x^{2}\left(\left[D_{Y}, \frac{T}{4}\right] F_{y}\right) E \\
& +x^{2} F_{y}\left(\widetilde{D}_{B}^{\prime \prime \prime} E\right)+x^{3}\left(\left[\widetilde{D}_{B}, \frac{T}{4}\right] F_{y}\right) E+x^{3} F_{y}\left(\left[\widetilde{D}_{B}, \frac{T}{4}\right] E\right)+x^{4}\left(\frac{T}{4}\right)^{2} F_{y} E \\
= & x^{2}\left(\widetilde{D}_{B}^{2} F_{y}\right) E+x^{2}\left(\widetilde{D}_{B}^{\prime} F_{y}\right)\left(\widetilde{D}_{B}^{\prime \prime} E\right)+x^{2} F_{y}(R E)+x^{2}\left(\left[D_{Y}, \frac{T}{4}\right] F_{y}\right) E \\
& +x^{2} F_{y}\left(\widetilde{D}_{B}^{\prime \prime \prime} E\right)+x^{3}\left(\left[\widetilde{D}_{B}, \frac{T}{4}\right] F_{y}\right) E+x^{3} F_{y}\left(\left[\widetilde{D}_{B}, \frac{T}{4}\right] E\right)+x^{4}\left(\frac{T}{4}\right)^{2} F_{y} E .
\end{aligned}
$$

Here $\widetilde{D}_{B}^{\prime}$ etc. are first order differential operators acting horizontally, and the curvature factor $R$ comes from the use of the Lichnerowicz formula. 
To estimate (3.5), we introduce the following notation. We use ||$_{\infty}$ to denote the sup norm, and ||$_{\infty, i}$ the sup norm of all the derivatives up to $i$ th order. Here we make the convention that derivatives are taken with respect to the space variables but not the time variable. Thus $\left|F_{y}\left(t, x, z, z^{\prime}\right)\right|_{\infty, i_{1}, i_{2}, i_{3}}$ denotes the sup norm of $F$ together with its derivatives with respect to $y$ up to $i_{1}$ th order, $z$ up to $i_{2}$ th order, $z^{\prime}$ up to $i_{3}$ th order.

First we show that for $t \geq 1$,

$$
\left|F_{y}\left(t, x, z, z^{\prime}\right)\right|_{\infty, i_{1}, i_{2}, i_{3}} \leq C\left(i_{1}, i_{2}, i_{3}\right) .
$$

In fact, for $i_{1}=0$, this is the standard heat kernel estimate and follows from, for example, [CGT]. For arbitrary $i_{1}$, note that, since $\operatorname{dim} \operatorname{ker} D_{Y}$ is assumed to be a (finite) constant and $D_{Y}^{2}+x\left[D_{Y}, \widetilde{D}_{B}\right]$ is a small perturbation of $D_{Y}^{2}$, it follows that all its eigenvalues are bounded away from 0 by a positive constant $\lambda_{0}$ except a finite number of them, which decay in $x$. We claim that such eigenvalues must be decaying quadratically. To see this, note that in terms of the splitting

$$
L^{2}(Y)=\operatorname{ker} D_{Y} \oplus\left(\operatorname{ker} D_{Y}\right)^{\perp},
$$

our operator $D_{Y}^{2}+x\left[D_{Y}, \widetilde{D}_{B}\right]$ is of the following special form

$$
D_{Y}^{2}+x\left[D_{Y}, \widetilde{D}_{B}\right]=\left(\begin{array}{cc}
0 & x C \\
x C^{*} & D
\end{array}\right),
$$

where $C=P_{\operatorname{ker} D_{Y}}\left[D_{Y}, \widetilde{D}_{B}\right] P_{\operatorname{ker} D_{Y}}^{\perp}$ is bounded (cf. Lemma 2.6), and $D=$ $P_{\mathrm{ker} D_{Y}}^{\perp}\left(D_{Y}^{2}+x\left[D_{Y}, \widetilde{D}_{B}\right]\right) P_{\mathrm{ker} D_{Y}}^{\perp}$. Our claim follows from a similar analysis as in the proof of (1.11).

Let $P$ be the projection on the space spanned by the eigenspaces corresponding to such eigenvalues. $\mathrm{P}$ is a smoothing operator that depends smoothly also on the base variable. Let $F_{y}^{0}\left(t, x, z, z^{\prime}\right)$ denote the Schwartz kernel of $P F_{y} P$. $F_{y}^{0}\left(t, x, z, z^{\prime}\right)$ obeys

$$
\left|F_{y}^{0}\left(t, x, z, z^{\prime}\right)\right|_{\infty, i_{1}, i_{2}, i_{3}} \leq C\left(i_{1}, i_{2}, i_{3}\right) .
$$

Put $\underline{D}_{Y}=\left.\left(D_{Y}^{2}+x\left[D_{Y}, \widetilde{D}_{B}\right]\right)\right|_{P^{\perp}}$. Then its eigenvalues are bounded away from 0 by $\lambda_{0}$. Therefore, if $F_{y}\left(t, x, z, z^{\prime}\right)$ denotes the heat kernel of $\underline{D}_{Y}$, for $t \geq 1$,

$$
\left|\bar{F}_{y}\left(t, x, z, z^{\prime}\right)\right|_{\infty, 0, i_{2}, i_{3}} \leq C\left(i_{2}, i_{3}\right) e^{-t \lambda_{0}}
$$

Now by Duhamel's principle,

$$
\frac{\partial}{\partial y} \bar{F}_{y}\left(t, x, z, z^{\prime}\right)=-\int_{0}^{t} \int_{Y} \bar{F}_{y}\left(s, x, z, z^{\prime \prime}\right) \frac{\partial \underline{D}_{Y}}{\partial y} \bar{F}_{y}\left(t-s, x, z^{\prime \prime}, z^{\prime}\right) d z^{\prime \prime} d t .
$$

To estimate this convolution, we consider the time intervals $s \leq t / 2$ and $s \geq t / 2$ separately. If $s \leq t / 2$, then $t-s \geq t / 2$. Thinking of $\bar{F}_{y}\left(s, x, z, z^{\prime \prime}\right)$ as the kernel of an integral operator on $L^{2}(Y)$ whose norm is bounded by 1 , we see 
that by the elliptic estimate,

$$
\begin{aligned}
& \left|\int_{Y} \bar{F}_{y}\left(s, x, z, z^{\prime \prime}\right) \frac{\partial \underline{D}_{Y}}{\partial y} \bar{F}_{y}\left(t-s, x, z^{\prime \prime}, z^{\prime}\right) d z^{\prime \prime}\right|_{\infty, 0, i_{2}, i_{3}} \\
& \quad \leq\left\|\underline{D}_{Y, 1}^{i_{2}} \underline{D}_{Y, 2}^{i_{3}} \frac{\partial \underline{D}_{Y}}{\partial y} \bar{F}_{y}\left(t-s, x, z^{\prime \prime}, z^{\prime}\right)\right\|_{L^{2}(Y)} \\
& \quad \leq C\left|\bar{F}_{y}\left(t-s, x, z^{\prime \prime}, z^{\prime}\right)\right|_{\infty, 0,2 i_{2}+2,2 i_{3}} \leq C\left(i_{2}, i_{3}\right) e^{-t \lambda_{0}} .
\end{aligned}
$$

On the other hand, if $s \geq t / 2$, note that

$$
\int_{Y} \bar{F}_{y}(s) \frac{\partial \underline{D}_{Y}}{\partial y} \bar{F}_{y}(t-s) d z^{\prime \prime}=\int_{Y}\left(\frac{\partial \underline{D}_{Y}}{\partial y}\right)^{*} \bar{F}_{y}(s) \bar{F}_{y}(t-s) d z^{\prime \prime} .
$$

Thus letting $\bar{F}_{y}\left(t-s, x, z^{\prime \prime}, z^{\prime}\right)$ play the role of $\bar{F}_{y}\left(s, x, z, z^{\prime \prime}\right)$, we obtain the same estimate. Hence

$$
\left|\frac{\partial}{\partial y} \bar{F}_{y}\left(t, x, z, z^{\prime}\right)\right|_{\infty, 0, i_{2}, i_{3}} \leq C\left(i_{2}, i_{3}\right) t e^{-t \lambda_{0}},
$$

or

$$
\left|\bar{F}_{y}\left(t, x, z, z^{\prime}\right)\right|_{\infty, 1, i_{2}, i_{3}} \leq C\left(i_{2}, i_{3}\right)
$$

Proceed inductively, one proves that

$$
\left|\bar{F}_{y^{\prime}}\left(t, x, z, z^{\prime}\right)\right|_{\infty, i_{1}, i_{2}, i_{3}} \leq C\left(i_{1}, i_{2}, i_{3}\right) \text {. }
$$

Combined with (3.6), this gives the estimate we want.

Second, for $t x^{2}$ small, note the standard heat kernel estimate, which follows from the finite propagation speed technique [CGT],

$$
\left|E\left(t x^{2}, y, y^{\prime}\right)\right|_{\infty, i_{1}, i_{2}} \leq C\left(i_{1}, i_{2}\right)\left(t x^{2}\right)^{-n / 2-i_{1} / 2-i_{2} / 2} .
$$

From these discussions we see by (3.5)

$$
\left|G_{x}\right| \leq C\left(t x^{2}\right)^{-n / 2-1 / 2} x^{2}, \quad \text { for } t \geq 1 .
$$

Denote

$$
G_{x} \# G_{x}=-\int_{0}^{t} \int G_{x}\left(s, y, z, y^{\prime}, z^{\prime}\right) G_{x}\left(t-s, y^{\prime}, z^{\prime}, y^{\prime \prime}, z^{\prime \prime}\right) d y^{\prime} d z^{\prime} d s .
$$

In general denote

$$
G_{x}^{m}=\underbrace{G_{x} \# \ldots \# G_{x}}_{m+1} .
$$

A version of Duhamel's principle says

$$
e^{-t D_{x}^{2}}=\sum_{i=0}^{m-1}(-1)^{i+1} G_{x}^{i} \# K_{x}+G_{x}^{m} \# e^{-t D_{x}^{2}} .
$$

Applying this to get the asymptotic expansion, we need estimates on the $m$-fold convolutions $G_{x}^{m}$. 
Lemma 3.5. $G_{x}^{m}$ improves with $m$,

$$
\left|G_{x}^{m}\right| \leq C(m)\left(t x^{2}\right)^{-n / 2+(m-1) / 2} x^{2} .
$$

Proof. Let us begin with $G_{x} \# G_{x}$. We divide the integral over $[0, t]$ into two, one over $[0, t / 2]$, and the other $[t / 2, t]$,

$$
\begin{aligned}
G_{x} \# G_{x}= & -\int_{0}^{t / 2} \int G_{x}\left(s, y, z, y^{\prime}, z^{\prime}\right) G_{x}\left(t-s, y^{\prime}, z^{\prime}, y^{\prime \prime}, z^{\prime \prime}\right) d y^{\prime} d z^{\prime} d s \\
& -\int_{t / 2}^{t} \int G_{x}\left(s, y, z, y^{\prime}, z^{\prime}\right) G_{x}\left(t-s, y^{\prime}, z^{\prime}, y^{\prime \prime}, z^{\prime \prime}\right) d y^{\prime} d z^{\prime} d s \\
& \stackrel{\text { def }}{=} \mathrm{I}+\mathrm{II} .
\end{aligned}
$$

By (3.5) and integration by parts, we can rewrite I as

$$
I=-\int_{0}^{t / 2} \int K_{x}\left(s, y, z, y^{\prime}, z^{\prime}\right) G_{x}^{\prime}\left(t-s, y^{\prime}, z^{\prime}, y^{\prime \prime}, z^{\prime \prime}\right) d y^{\prime} d z^{\prime} d s,
$$

where by the same consideration as in showing (3.7)

$$
\left|G_{x}^{\prime}\right| \leq C\left(t x^{2}\right)^{-n / 2-1} x^{4}
$$

As an integral operator on the $L^{2}$ space, the norm of $K_{x}$ is bounded by 1 . Therefore,

$$
\begin{aligned}
|I| & \leq t / 2\left\|G_{x}^{\prime}\left(t-s, \cdot, \cdot, y^{\prime \prime}, z^{\prime \prime}\right)\right\|_{2} \\
& \leq C\left(t x^{2}\right)^{-n / 2} x^{2} .
\end{aligned}
$$

The estimate for II is similar. In this way one obtains

$$
\left|G_{x} \# G_{x}\right| \leq C\left(t x^{2}\right)^{-n / 2+1} \text {. }
$$

In estimating the $m$-fold convolution, one divides the time interval into $m$ subintervals, on each of them one of the $m$-tuplet $t_{0}-t_{1}, \ldots, t_{m-2}-t_{m}$ is greater than or equal to $t / m$. One lets the kernels corresponding to the other time parameters play the role of $K$ (after integration by parts), and proceeds as for $G_{x} \# G_{x}$.

Now by the standard heat asymptotic expansion and the above estimate, we arrive at the uniform asymptotic expansion as claimed.

\section{THE SIGNATURE OPERATOR AND HODge-LERAY THEORY}

In the previous sections we proved the adiabatic limit formula (0.1) with the global contribution expressed in terms of the very small eigenvalues. Here, confining ourselves to signature operators, we identify this global contribution with a topological invariant constructed from the Leray spectral sequence of the fibration. In fact we show that one can recover the Leray spectral sequence from the eigenspaces associated to these eigenvalues. 
4.1. The signature operator on an odd-dimensional manifold. For an even-dimensional oriented Riemannian manifold $N^{n}$, one can define an involution $\tau$ on the complexified exterior bundle $\Lambda^{*} N \otimes \mathbb{C}$ by setting

$$
\left.\tau\right|_{\Lambda^{p}}=(\sqrt{-1})^{\frac{n}{2}+p(p-1)} * \text {. }
$$

Correspondingly $\Lambda^{*} N \otimes \mathbb{C}$ decomposes into the \pm 1 -eigenspaces of $\tau$ :

$$
\Lambda^{*} N \otimes \mathbb{C} \simeq \Lambda_{+} \oplus \Lambda_{-}
$$

The operator

$$
d+\delta: \Lambda^{*} N \otimes \mathbb{C} \rightarrow \Lambda^{*} N \otimes \mathbb{C}
$$

anticommutes with $\tau$ and hence

$$
(d+\delta)_{+}: \Lambda_{+} \rightarrow \Lambda_{-}
$$

This operator is called the signature operator on $N$ for the reason that its index gives the signature of $N$ when $N$ is compact.

When $N$ is spin, one has the bundle isomorphism

$$
\Lambda^{*} N \otimes \mathbb{C} \simeq F(N) \otimes F(N) .
$$

This is basically because the twisted adjoint representation is equivalent to the tensor product of the spinor representation with itself, see [Gi, Chapter 3.2; LM, Chapter 1].

It is important to note that the two Clifford module structures correspond to multiplication on the left and on the right (by the transpose) in $\mathrm{Cl}(n)$. It is not hard to verify that under (4.1)

$$
\begin{aligned}
& e \cdot \varphi \simeq\left(\operatorname{ext}_{e}-\text { int }_{e}\right) \varphi, \\
& \varphi \cdot e \simeq\left(\operatorname{ext}_{e}+\text { int }_{e}\right) \alpha \varphi,
\end{aligned}
$$

for $e \in T N$. Here ext ${ }_{e}$ and int ${ }_{e}$ denote the exterior and interior multiplication respectively, and $\left.\alpha\right|_{\Lambda^{p}}=(-1)^{p}$.

With the bundle isomorphism (4.1) one has

$$
d+\delta \cong D \otimes F(N),
$$

where the connections on both the left and the right $F(N)$ are induced by the Levi-Civita connection on $N$. Moreover the involution $\tau$ also identifies with the involution on the left $F(N)$ that determines the splitting

$$
F(N)=F_{+}(N) \oplus F_{-}(N) .
$$

It follows that

$$
(d+\delta)_{+} \cong D_{+} \otimes F(N)
$$

For an oriented odd-dimensional manifold $M^{n}$, we define the signature operator $A$ to be the tangential part of the signature operator on $M \times \mathbb{R}_{+}$. Here 
$\mathbb{R}_{+}=[0, \infty)$ and $M \times \mathbb{R}_{+}$is given the product metric $d r^{2}+g_{M}$. Thus $A$ is such that

$$
(d+\delta)_{+}=\left(\operatorname{ext}_{\partial_{r}}-\text { int }_{\partial_{r}}\right)\left(\partial_{r}+A\right),
$$

and $A$ is a selfadjoint operator acting on $\Lambda_{+}\left(M \times \mathbb{R}_{+}\right)$restricted to $M$.

Now restricted to $M, \Lambda_{+}\left(M \times \mathbb{R}_{+}\right)$can be identified with $\Lambda^{*} M \otimes \mathbb{C}$. A straightforward calculation yields

$$
A=\tau(d+\delta)=d \tau+\tau d: \Lambda^{*} M \otimes \mathbb{C} \rightarrow \Lambda^{*} M \otimes C,
$$

where $\left.\tau\right|_{\Lambda^{p}}=(\sqrt{-1})^{\frac{n+1}{2}+p(p-1)} *, \tau^{2}=1$.

On the other hand, by using (4.4), one finds

$$
A \cong D \otimes F(M \times \mathbb{R}): F(M) \otimes F(M \times \mathbb{R}) \rightarrow F(M) \otimes F(M \times \mathbb{R}) .
$$

Here the identification is

$$
\begin{aligned}
\Lambda^{*} M \simeq \Lambda_{+}\left(M \times \mathbb{R}_{+}\right) & \simeq F_{+}(M \times \mathbb{R}) \otimes F(M \times \mathbb{R}) \\
& =F(M) \otimes F(M \times \mathbb{R}) .
\end{aligned}
$$

One verifies that (4.2) and (4.3) still hold.

Suppose now that $M$ is fibered by (1.1), where we assume the base $B$, and the vertical bundle $T^{V} M$ are both oriented. (In this case we say the fibration is oriented.) In view of (4.5) one would like to apply Theorem 0.1 to the operator $A_{x}$. There is a minor complication here since in (4.5) we are forced to use on the twisted bundle $F(M \times \mathbb{R})$ the connection induced by the LeviCivita connection of the rescaled metric $g_{x}$, rendering it $x$-dependent. Now $\nabla^{L, x}=\nabla+S^{x}$. By a straightforward computation one finds

$$
\begin{aligned}
A_{x}= & A_{Y}+x\left[A_{B} \otimes 1+f_{\alpha} \otimes \nabla_{f_{\alpha}}+\frac{1}{2}\left\langle S\left(e_{i}\right) e_{j}, f_{\alpha}\right\rangle e_{i}\left(e_{j} f_{\alpha}+e_{j}^{*} f_{\alpha}^{*}\right)\right] \\
& +x^{2} \frac{1}{4}\left\langle S\left(e_{i}\right) f_{\alpha}, f_{\beta}\right\rangle\left(-e_{i} f_{\alpha} f_{\beta}+e_{i} f_{\alpha}^{*} f_{\beta}^{*}+2 f_{\alpha} e_{i}^{*} f_{\beta}^{*}\right) .
\end{aligned}
$$

Here the $e_{i}$ 's and $f_{\alpha}$ 's are left Clifford multiplications acting by (4.2) and the $e_{i}^{*}$ 's and $f_{\alpha}^{*}$ 's are right Clifford multiplications acting by (4.3). Also $A_{Y}$ is the family of signature operators along the fibers, $A_{B}$ the signature operator on the base, and the summation convention is understood.

Since in Getzler's transformation, we only scale the "left" Clifford variables but not the "right" Clifford variables, it is clear that the extra terms in (4.6) (i.e. those involving the right Clifford variables ) do not contribute to the first term in the adiabatic limit formula. That is, for the first term (the $\tilde{\eta}$ term), it is as if we are dealing with $D \otimes F(M \times \mathbb{R})$ where the connection on the twisting bundle $F(M \times \mathbb{R})$ is induced by $\nabla$ (which does not depend on $x$ ).

For the second term (the twisted $\eta$ term), however, these terms do make a difference. Let

$A_{B} \otimes \operatorname{ker} A_{Y}=P_{\operatorname{ker} A_{Y}}\left[A_{B} \otimes 1+f_{\alpha} \otimes \nabla_{f_{\alpha}}+\frac{1}{2}\left\langle S\left(e_{i}\right) e_{j}, f_{\alpha}\right\rangle e_{i}\left(e_{j} f_{\alpha}+e_{j}^{*} f_{\alpha}^{*}\right)\right] P_{\operatorname{ker} A_{Y}}$. 
Then

Corollary 4.1. The adiabatic limit of the $\eta$-invariant of the signature operator always exists. Moreover,

$$
\lim _{x \rightarrow 0} \eta\left(A_{x}\right)=2 \int_{B} \mathscr{L}\left(\frac{R^{B}}{2 \pi}\right) \wedge \tilde{\eta}+\eta\left(A_{B} \otimes \operatorname{ker} A_{Y}\right)+\lim _{x \rightarrow 0} \sum_{\lambda_{0}, \lambda_{1}=0} \operatorname{sgn} \lambda_{x} .
$$

Proof. By the Hodge theorem, both $\operatorname{ker} A_{x}$ and $\operatorname{ker} A_{Y}$ are of constant dimension. Therefore this is an immediate consequence of the above discussion and the proof of Theorem 0.1 .

Before we go into the topological interpretation of the very small eigenvalues, we give two ways of thinking about the operator $A_{B} \otimes \operatorname{ker} A_{Y}$. First note that

$$
\nabla_{f_{\alpha}}-\frac{1}{2}\left\langle S\left(e_{i}\right) e_{i}, f_{\alpha}\right\rangle
$$

defines a unitary connection with respect to the $L^{2}$-metric on the infinitedimensional bundle over $B$ of the differential forms along the fibers, cf. $\S 1.1$. From here one sees that $A_{B} \otimes \operatorname{ker} A_{Y}$ is the sum of the signature operator on the base coupled to the projected unitary connection on $\operatorname{ker} A_{Y}$ and $\frac{1}{2}\left\langle S\left(e_{i}\right) e_{j}, f_{\alpha}\right\rangle$ $\times P_{\text {ker } A_{Y}} e_{i} e_{j}^{*} f_{\alpha}^{*} P_{\operatorname{ker} A_{Y}}$.

The operator $A_{B} \otimes \operatorname{ker} A_{Y}$ can also be expressed in terms of the natural flat connection on $\operatorname{ker} A_{Y}\left(=\mathscr{H}^{*}(Y)\right)$. Let $d$ be the exterior differential on the $\left(\operatorname{ker} A_{Y}\right)$-valued differential forms defined in terms of the flat connection. We define an involution $\tau$ on $\Lambda^{*}(B) \otimes \operatorname{ker} A_{Y}$ by

$$
\tau=P_{\text {ker } A_{Y}}(\sqrt{-1})^{\frac{n+1}{2}} f_{1} \cdots f_{k} e_{1} \cdots e_{l} P_{\operatorname{ker} A_{Y}}
$$

This is essentially the Hodge $*$ operator on the base coupled to the $*$ operators along the fibers. Then

$$
A_{B} \otimes \operatorname{ker} A_{Y}=d \tau+\tau d
$$

To see this, define a new connection $\nabla^{Y}$ on $T^{V} M$ by setting

$$
\nabla_{U_{2}}^{Y} U_{1}=P^{V}\left(\nabla_{U_{2}}^{L} U_{1}\right), \quad \nabla_{U}^{Y} X=[U, X]
$$

The projection of $\nabla^{Y}$ is the flat connection on $\operatorname{ker} A_{Y}$. Let

$$
\tilde{d}=f_{\alpha} \wedge\left(\nabla_{f_{\alpha}}^{B} \otimes 1+1 \otimes \nabla_{f_{\alpha}}^{Y}\right), \quad \tilde{\tau}=(\sqrt{-1})^{\frac{n+1}{2}} f_{1} \cdots f_{k} e_{1} \cdots e_{l},
$$

and

$$
\tilde{A}=\tilde{d} \tilde{\tau}+\tilde{\tau} \tilde{d}
$$

It can be shown that

$$
d \tau+\tau d=P_{\text {ker } A_{Y}} \widetilde{A} P_{\text {ker } A_{Y}}
$$


and by a straightforward calculation using (4.2) and (4.3) one finds

$$
A_{B} \otimes 1+f_{\alpha} \otimes \nabla_{f_{\alpha}}+\frac{1}{2}\left\langle S\left(e_{i}\right) e_{j}, f_{\alpha}\right\rangle e_{i}\left(e_{j} f_{\alpha}+e_{j}^{*} f_{\alpha}^{*}\right)=\tilde{A} .
$$

Our claim follows.

4.2. Hodge-Leray theory of the very small eigenvalues. Here we start out to prove Theorem 0.2. We use Serre's construction of the Leray spectral sequence. To relate our space $E_{r}$ to the $E_{r}$-term of the Leray spectral sequence, we introduce another complex that incorporates the asymptotic properties in its filtration. Let us first recall Serre's filtration.

Consider the de Rham complex $\left(C^{\infty}\left(\Lambda^{*} M\right), d\right)$. An $l$-form $\omega$ is in $F^{i}$ if

$$
\omega\left(U_{1}, U_{2}, \ldots, U_{l}\right)=0,
$$

whenever $l-i+1$ of the tangent vectors $U_{j}$ 's are vertical.

In our case, we are given a splitting $T M=T^{H} M \oplus T^{V} M \cong \pi^{*} T B \oplus T^{V} M$. Thus $\Lambda^{*} M=\pi^{*} \Lambda^{*} B \otimes \Lambda^{*} T^{V} M$. Formally, we can use $a(y, z) d y^{\alpha} \wedge d z^{\beta}(y$ local coordinates of $B, z$ local coordinates of $Y$ ) to indicate such a splitting. With this convention, Serre's filtration can be simply described as

$$
F^{i}=\left\{a(y, z) d y^{\alpha} \wedge d z^{\beta}:|\alpha| \geq i\right\} .
$$

It is well known that Serre's filtration gives rise to the Leray spectral sequence of the fibration, $\left(\bar{E}_{i}, \bar{d}_{i}\right)$.

To make sense of the spaces considered in the Hodge-Leray theorem (Theorem 0.2 ) we proceed to construct good bases for such spaces. These bases are also used in proving the Hodge-Leray theorem. Let us first introduce some notations.

Let $\Lambda_{k}$ denote $k$-tuplets of real numbers

$$
\Lambda_{k}=\left\{\lambda_{0}, \lambda_{1}, \ldots, \lambda_{k-1}\right\}
$$

and similarly we use $\Lambda$ to denote infinite sequence of real numbers

$$
\Lambda=\left\{\lambda_{0}, \lambda_{1}, \ldots\right\} .
$$

Also, let $\lambda_{x}^{\prime}$ be a function of $x$ that has complete asymptotic expansion at $x=0$. When we say $\lambda_{x}^{\prime} \in \Lambda_{k}$ we mean that the first $k$ coefficients in the asymptotic expansion of $\lambda_{x}^{\prime}$ at $x=0$ are prescribed by $\Lambda_{k}$

$$
\lambda_{x}^{\prime} \sim \lambda_{0}+\lambda_{1} x+\cdots+\lambda_{k-1} x^{k-1}+\cdots .
$$

Similarly $\lambda_{x}^{\prime} \in \Lambda$ means all the coefficients are prescribed by $\Lambda$. Now for the eigenvalues $\lambda_{x}$ of $A_{x}$, set $\lambda_{x}^{\prime}=\lambda_{x} / x$ and define

$$
G_{\Lambda_{k}}=\bigoplus_{\lambda_{x}^{\prime} \in \Lambda_{k}} E\left(\lambda_{x}\right), \quad G_{\Lambda}=\bigoplus_{\lambda_{x}^{\prime} \in \Lambda} E\left(\lambda_{x}\right),
$$


where $E\left(\lambda_{x}\right)$ is the eigenspace associated to $\lambda_{x}$. Thus $G_{\Lambda_{k}}$ is the direct sum of the eigenspaces corresponding to the eigenvalues whose first coefficient in the asymptotic expansion is 0 and whose next $k$ coefficients are prescribed by $\Lambda_{k}$, and $G_{\Lambda}$ is the direct sum of the eigenspaces corresponding to the eigenvalues whose first coefficient is 0 and whose other coefficients are all prescribed by $\Lambda$. Obviously

$$
G_{\Lambda} \subset G_{\Lambda_{k}} \subset G_{\Lambda_{k-1}}
$$

Proposition 4.2. Let $\lambda_{x}$ be an eigenvalue of $A_{x}$ such that $\lambda_{x} \sim \lambda_{1} x+\lambda_{2} x^{2}+$ $\ldots$, and let $\Lambda$ be the infinite sequence given by the coefficients of $\lambda_{x}$. Then $G_{\Lambda}$ is a family of finite-dimensional vector space that depends smoothly on $x$ down to $x=0$. That is, there is an orthonormal basis for $G_{\Lambda}$ that is smooth in $x$ down to $x=0$.

Proof. The same analysis as in the proof of Theorem 1.5 reduces it to the finite-dimensional case. Therefore it suffices to prove the following lemma

Lemma 4.3. If $T(x)$ is a family of finite-dimensional selfadjoint operators that depends smoothly on $x$, then $G_{\Lambda_{k}}=\bigoplus_{\lambda_{x} \in \Lambda} E\left(\lambda_{x}\right)$ depends smoothly on $x$ for all $k=1,2, \ldots, \infty \quad\left(\Lambda_{\infty}=\Lambda\right)$.

Proof. Note the slight modification in the definition of $G_{\Lambda_{k}}$. The idea is to show by induction that the orthogonal projection $Q_{k}(x)$ on $G_{\Lambda_{k}}$ depends smoothly on $x$. Then by Lemma 2.9 , we have a smooth family of unitary operators $V_{k}(x)$ such that

$$
V_{k}(x)^{-1} Q_{k}(x) V_{k}(x)=Q_{k}(0) \text {. }
$$

Therefore the desired basis can be gotten by taking the $V_{k}(x)$-image of an orthonormal basis for Image $Q_{k}(0)$.

By the discreteness of $\operatorname{spec}(T(0))$, there exists an $\varepsilon>0$ such that $\lambda_{0}$ is the only point of $\operatorname{spec}(T(0))$ in $\left|\lambda-\lambda_{0}\right|<\varepsilon$. By the first resolvent formula and the smoothness of $T(x)$,

$$
Q(x)=\frac{1}{2 \pi i} \int_{\left|\lambda-\lambda_{0}\right|=\varepsilon}(T(x)-\lambda)^{-1} d \lambda
$$

is well defined for small $x$ and is smooth. One easily sees that $Q(x)=Q_{1}(x)$.

Now assume that $Q_{k-1}(x)$ is smooth. Then the family

$$
S(x) \stackrel{\text { def }}{=} V_{k-1}(x)^{-1} T(x) V_{k-1}(x)
$$

is smooth on Image $Q_{k-1}(0)$ and the eigenvalues of $S(x)$ are the eigenvalues of $T(x)$ having the first $k-1$ coefficients of their asymptotic expansions prescribed by $\Lambda_{k-1}$. Set

$$
R(x)=\frac{S(x)-\left(\lambda_{0}+\lambda_{1} x+\cdots+\lambda_{k-1} x^{k-1}\right)}{x^{k}} .
$$


This is a smooth family of selfadjoint operators, and as above, one finds

$$
V_{k-1}(x)^{-1} Q_{k}(x) V_{k-1}(x)=\frac{1}{2 \pi i} \int_{\left|\lambda-\lambda_{k}\right|=\varepsilon}(R(x)-\lambda)^{-1} d \lambda .
$$

This shows that $Q_{k}(x)$ is also smooth and the induction is complete.

Because we are in a finite-dimensional space, the induction process must stop in finitely many steps, proving it for $k=\infty$.

Now consider

$$
\Lambda_{r}^{0}=\{\underbrace{0,0, \ldots, 0}_{r-1}\}, \quad r \geq 2 .
$$

Then $G_{\Lambda_{r}^{0}}$ is the direct sum of the eigenspaces associated to eigenvalues that decay at least like $x^{r}$. From the previous discussion, one knows that $G_{\Lambda_{r}^{0}}$ is a smooth family of finite-dimensional vector spaces, thus it makes sense to consider

$$
E_{r}=\lim _{x \rightarrow 0} G_{\Lambda_{r}^{0}}
$$

for $r \geq 2$. This defines the space considered in Theorem 0.2 .

Before we introduce our next complex, recall that $X=[0, \infty) \times M$. Define the bundle ${ }^{\mathscr{V}} T^{*} M$ to be spanned by $\frac{d y}{x}, d z$. Its corresponding exterior bundle is denoted by ${ }^{\mathscr{V}} \Lambda^{*}$. The space of Laurent series, $\mathscr{L}\left(X,{ }^{\mathscr{V}} \Lambda^{*}\right)$ is defined as,

$$
\mathscr{L}\left(X,{ }^{\mathscr{V}} \Lambda^{*}\right)=\frac{\left\{u \in C^{\infty}\left(X,{ }^{\mathscr{V}} \Lambda^{*}\right), x^{q} u \in C^{\infty}\left(X,{ }^{\mathscr{V}} \Lambda^{*}\right), \text { for some } q \in Z\right\}}{\dot{C}^{\infty}\left(X,{ }^{\mathscr{V}} \Lambda^{*}\right)},
$$

where $\dot{C}^{\infty}\left(X,{ }^{\mathscr{V}} \Lambda^{*}\right)$ is the space of $C^{\infty}$ sections vanishing to all orders at the boundary. $\mathscr{L}\left(X,{ }^{\mathscr{V}} \Lambda^{*}\right)$ is a module over the ring of formal Laurent series

$$
\mathscr{L}=\mathscr{L}([0, \infty))
$$

in the variable $x$.

Formally, an element in $\mathscr{L}\left(X,{ }^{\mathscr{V}} \Lambda^{*}\right)$ can be represented as $\sum_{j \geq i} a_{j} x^{j}$, where $a_{i}$ is sum of elements of the form $b(y, z)\left(\frac{d y}{x}\right)^{\alpha} d z^{\beta}$ with $b$ a smooth function. The exterior differential $d$ acts on it in the obvious way.

Now consider the complex $\left(\mathscr{L}\left(X,{ }^{\mathscr{V}} \Lambda^{*}\right), d\right)$. It comes equipped with a natural filtration $F^{i}=\left\{\sum_{j \geq i} a_{j} x^{j}\right\}$, thus giving rise to a spectral sequence $\left(\widetilde{E}_{i}, \tilde{d}_{i}\right)=\left(\bigoplus_{p} \widetilde{E}_{i}^{p}, \bigoplus_{p} \tilde{d}_{i}^{p}\right)$. Note that $x^{q}: F^{i} \cong F^{i+q}$ induces $\widetilde{E}_{i}^{p} \cong \widetilde{E}_{i}^{p+q}$. Further the diagram

$$
\begin{array}{lll}
\widetilde{E}_{i}^{p} & \stackrel{\tilde{d}_{i}^{p}}{\longrightarrow} & \widetilde{E}_{i}^{p+i} \\
\downarrow x^{q} & & \downarrow x^{q} \\
\widetilde{E}_{i}^{p+q} & \stackrel{\tilde{d}_{i}^{p+q}}{\longrightarrow} & \widetilde{E}_{i}^{p+q+i}
\end{array}
$$

commutes: $x^{q} \tilde{d}_{i}^{p}=\tilde{d}_{i}^{p+q} x^{q}$. Hence $\widetilde{E}_{i} \cong \widetilde{E}_{i}^{0} \otimes \mathscr{L}(x)$ (with the natural gradation induced by that of $\mathscr{L}(x))$ and $\tilde{d}_{i}^{p} \cong x^{p} \tilde{d}_{i}^{0} \otimes 1$. 
Explicit calculation shows

$$
\begin{aligned}
& \widetilde{E}_{0} \cong C^{\infty}\left(\Lambda^{*} B \otimes C^{\infty}\left(\Lambda^{*} Y\right)\right) \otimes \mathscr{L}(x), \\
& \widetilde{E}_{1} \cong C^{\infty}\left(\Lambda^{*} B \otimes \mathscr{H}^{*}(Y)\right) \otimes \mathscr{L}(x), \\
& \widetilde{E}_{2} \cong H\left(B, \mathscr{H}^{*}(Y)\right) \otimes \mathscr{L}(x) .
\end{aligned}
$$

By the finite dimensionality of $\widetilde{E}_{2}$ (as an $\mathscr{L}(x)$-module), this spectral sequence must degenerate after finitely many steps.

Remark. These quotient modules ( $\mathscr{L}(x)$-modules) can actually be viewed as vector spaces.

Proof of Theorem 0.2. We prove $E_{r} \cong \bar{E}_{r}$ by showing that both of them are isomorphic to $\widetilde{E}_{r}^{0}$.

(a) $\bar{E}_{r} \cong \widetilde{E}_{r}^{0}$ for all $r \geq 0$.

Consider the natural inciusion:

$$
\begin{gathered}
C^{\infty}\left(\Lambda^{*} M\right) \\
\hookrightarrow \mathscr{L}\left(X,{ }^{\mathscr{V}} \Lambda^{*}\right), \\
a(y, z) d y^{\alpha} d z^{\beta} \rightarrow x^{|\alpha|} a(y, z)(d y / x)^{\alpha} d z^{\beta} .
\end{gathered}
$$

It is clearly filtration preserving. Thus it induces homomorphisms $l_{r}^{q}: \bar{E}_{r}^{q} \rightarrow \widetilde{E}_{r}^{q}$. Define

$$
\iota_{r}: \bar{E}_{r}=\bigoplus_{q=0}^{p} \bar{E}_{r}^{q} \stackrel{\oplus r_{r}^{q}}{\longrightarrow} \bigoplus_{q=0}^{p} \widetilde{E}_{r}^{q} \stackrel{\sum_{q} x^{-q}}{\longrightarrow} E_{r}^{0},
$$

i.e. $l_{r}=\sum_{q=0}^{p} x^{-q} l_{r}^{q}(p=\operatorname{dim} B)$. We show that $l_{r}$ is a homomorphism that preserves the differentials.

In fact we know that

$$
\begin{array}{lll}
\bar{E}_{r}^{p} & \stackrel{\bar{d}_{r}^{p}}{\longrightarrow} & \bar{E}_{r}^{p+r} \\
\downarrow l_{r}^{p} & & \downarrow l_{r}^{p+r} \\
\widetilde{E}_{r}^{p} & \stackrel{\tilde{d}_{r}^{p}}{\longrightarrow} & \widetilde{E}_{r}^{p+r}
\end{array}
$$

commutes. Hence $v_{r}^{p+r} \bar{d}_{r}^{p}=\tilde{d}_{r}^{p} l_{r}^{p}$. Also $x^{q} \tilde{d}_{i}^{p}=\tilde{d}_{i}^{p+q} x^{q}$. Therefore

$$
x^{-p-r} l_{r}^{p+r} \bar{d}_{r}^{p}=x^{-p-r} \tilde{d}_{r}^{p} l_{r}^{p}=\tilde{d}_{r}^{0} x^{-p} l_{r}^{p} .
$$

This shows

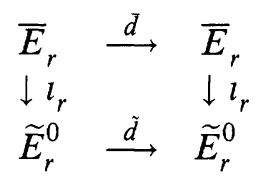

commutes, i.e. $l_{r} \bar{d}=\tilde{d}_{l_{r}}$.

Further, the explicit calculation (4.9) shows that $l_{r}$ induces isomorphisms for $r \leq 2$. Therefore it induces isomorphisms for all $\mathrm{r}$.

(b) $E_{r} \cong \widetilde{E}_{r}^{0}$ for $r \geq 2$. 
The scaled metric $g_{x}$ induces a bilinear form

$$
\mathscr{L}\left(X,{ }^{\mathscr{V}} \Lambda^{*}\right) \times \mathscr{L}\left(X,{ }^{\mathscr{V}} \Lambda^{*}\right) \stackrel{\langle,\rangle}{\longrightarrow} \mathscr{L}(x)
$$

and $\langle d \cdot, \cdot\rangle=\langle\cdot, \delta \cdot\rangle$. This is the same as saying that $\delta$ is the adjoint of $d$ with respect to $g_{x}$ for every $x$. (Here of course we are suppressing the $x$ dependence of $\delta$.) It follows that

$$
\|(d+\delta) z\|^{2}=\|d z\|^{2}+\|\delta z\|^{2} .
$$

Our operator $A_{x}$ is, up to sign, $*(d+\delta)$. Therefore if $A_{x} \varphi_{x}=\lambda_{x} \varphi_{x}$, then

$$
\left\|d \varphi_{x}\right\|^{2}+\left\|\delta \varphi_{x}\right\|^{2}=\left|\lambda_{x}\right|^{2}\left\|\varphi_{x}\right\|^{2}
$$

For simplicity we use the shorthand notation $z \sim x^{r}$ for $z \in F^{r}\left(\mathscr{L}\left(X,{ }^{\mathscr{V}} \Lambda^{*}\right)\right)$. Clearly $z \sim x^{r}$ is the same as $\|z\| \sim x^{r}$. By (4.10) one has

$$
(d+\delta) z \sim x^{r} \Longleftrightarrow d z \sim x^{r}, \quad \delta z \sim x^{r} .
$$

Basic to our proof is the observation that $\left(E_{r}, x^{-r} d\right)$ does form a spectral sequence, though it is not clear if it comes from a filtered complex. To see this, let us first show that $x^{-r} d$ maps $E_{r}$ into $E_{r}$. In fact, by the definition of $E_{r}$ and (4.11), (4.12), it suffices to show that $d$ leaves each eigenspace invariant. This can be seen in the following way. Clearly $d$ maps the 0-eigenspace to 0 (by, say, (4.10)). Now let $\lambda_{x} \neq 0$ be an eigenvalue of $A_{x}$ and $\varphi_{x}$ a corresponding eigenform, $A_{x} \varphi_{x}=\lambda_{x} \varphi_{x}$. But

$$
A_{x}= \pm * d \pm d *
$$

Thus

$$
\begin{aligned}
& \varphi_{x}=* d\left( \pm \varphi_{x} / \lambda_{x}\right)+d *\left( \pm \varphi_{x} / \lambda_{x}\right) \\
& \stackrel{\text { def }}{=} * d \psi_{x}+d * \psi_{x}^{\prime} .
\end{aligned}
$$

Since $* d \psi_{x}\left(d * \psi_{x}^{\prime}\right)$ is coexact (exact) and $A_{x}$ takes coexact (exact) forms to coexact (exact) forms, one must have

$$
\begin{aligned}
A_{x}\left(* d \psi_{x}\right) & =\lambda_{x}\left(* d \psi_{x}\right), \\
A_{x}\left(d * \psi_{x}^{\prime}\right) & =\lambda_{x}\left(d * \psi_{x}^{\prime}\right) .
\end{aligned}
$$

That is, the eigenspace corresponding to a nonzero eigenvalue splits into the direct sum of coexact and the exact eigenspaces. Obviously $d$ takes exact forms to zero. And (4.13) is the same as

$$
* d\left(* d \psi_{x}\right)=\lambda_{x}\left(* d \psi_{x}\right) .
$$

Taking $d$ of this equation one finds

$$
d * d\left(* d \psi_{x}\right)=\lambda_{x} d\left(* d \psi_{x}\right) \quad \text { or } \quad d *\left(d * d \psi_{x}\right)=\lambda_{x}\left(d * d \psi_{x}\right) .
$$

This proves the invariance of the $\lambda_{x}$-eigenspace under $d$. 
To see $E_{r+1} \cong H\left(E_{r}, x^{-r} d\right)$, note that by the finite-dimensional analog of the Hodge theory

$$
H\left(E_{r}, x^{-r} d\right) \cong \operatorname{ker}\left(x^{-r} d\right) \cap \operatorname{ker}\left(x^{-r} \delta\right) .
$$

Together with (4.11), this implies $\lambda_{x}=O\left(x^{r+1}\right)$ and the converse is easier.

Let $h_{r}=\operatorname{dim} E_{r}, h=\operatorname{dim} E_{\infty}=\operatorname{dim} H^{*}(M)$. By Proposition 4.2, we can choose

$$
\varphi_{1}, \ldots, \varphi_{h}, \ldots, \varphi_{h_{2}}
$$

such that $\varphi_{1}, \ldots, \varphi_{h_{r}}$ form a smooth basis for $E_{r}$, for all $r \geq 2$. From (4.12) it is clear that (4.14) induces a linear map

$$
E_{r} \longrightarrow \widetilde{E}_{r}^{0}
$$

It is a homomorphism between the two differential complex because the two differentials are formally identical. We show that it is an isomorphism by showing that it is an isomorphism for $r=2$.

1-1: If $z \in E_{r}$, then $d z \sim x^{r}, \delta z \sim x^{r}$. Assume $r \geq 1$. Now if $z=0$ in $\widetilde{E}_{r}^{0}$, then $z=z_{0}+d z_{1}$ with $z_{0} \sim x$ and $d z_{0} \sim x^{r}, z_{1} \sim x^{-r+1}, d z_{1} \sim x^{0}$. But then $\delta d z_{1}=\delta\left(z-z_{0}\right) \sim x$. Therefore $d z_{1} \sim x$ and thus $z \sim x$. i.e. $z=0$ in $E_{r}$.

onto : Let $z \in \widetilde{E}_{2}^{0}$. Then $z \sim x^{0}, d z \sim x^{2}$. Consider $z^{\prime}=z-$ $\sum_{i=1}^{h_{2}}\left\langle\varphi_{i}, z\right\rangle \varphi_{i}$. Clearly $z^{\prime} \perp E_{2}$ and $d z^{\prime} \sim x^{2}$. By the formal Hodge decomposition ([MM, Proposition (56)], or rather a straightforward adaptation to the operator $d+\delta$; cf. also its proof), $z^{\prime}=(d+\delta) z_{1}$ with $z_{1} \sim x^{-1}$. Now $d z^{\prime}=d \delta z_{1} \sim x^{2}$, which implies that $\delta z_{1} \sim x^{2}$, i.e. $\delta z_{1} \in Z_{1}^{1}$. Also $d z_{1} \in D_{1}^{0}$. Hence $z^{\prime}=0$ in $\widetilde{E}_{r}^{0}$. This shows that the map is onto for $r=2$.

Finally to show that the Hodge * operator with respect to $g_{x}$ induces the duality map on every $E_{r}$-term under the identification we just established, it suffices to prove it for the $E_{2}$-term because of the functoriality. Now if we realize $\bar{E}_{2}$ as $H^{*}\left(B, \mathscr{H}^{*}(Y)\right)$, i.e. the harmonic forms on the base with coefficients in the bundle of fiber-harmonic forms, the multiplication on $\bar{E}_{2}$ is just the wedge on the forms. Now by Lemma (49) in [MM] and the above discussion, we can actually deduce that

$$
H^{*}\left(B, \mathscr{H}^{*}(Y)\right)=E_{2}\left(\stackrel{\text { def }}{=} \lim _{x \rightarrow 0} G_{\Lambda_{2}^{0}}\right) .
$$

Our claim follows.

4.3. Adiabatic limit of $\eta$-invariant and nonmultiplicativity of signature. In $(0.1)$ the adiabatic limit formula, the global contribution is found to be $\sum_{\lambda_{0}, \lambda_{1}=0} \lim \operatorname{sgn} \lambda_{x}$. In the light of the Hodge-Leray theory proved above, it should be possible to characterize this term topologically. This is indeed the case here. 
To have a topological counterpart of this term, let us consider the Leray spectral sequence $\left(E_{r}, d_{r}\right)$. One has

$$
E_{2}=H^{*}\left(B, \mathscr{H}^{*}(Y)\right), \quad E_{\infty}=H^{*}(M) .
$$

Assume that our fibration is oriented. The orientation of $T^{V} M$ gives a trivialization of the flat line bundle $\mathscr{H}^{n-p}(Y)$ on $B$ (note that $n-p=\operatorname{dim} Y$ ). Together with the orientation of $B$, one finds

$$
E_{2}^{n}=H^{p}\left(B, \mathscr{H}^{n-p}(Y)\right) \cong H^{p}(B) \cong \mathbb{R} .
$$

Thus the multiplicative structure on $E_{2}$ gives rise to a Poincaré pairing

$$
E_{2}^{k} \otimes E_{2}^{n-k} \longrightarrow \mathbb{R}
$$

If we denote by $\xi_{2}$ the natural base for $E_{2}^{n}$ induced by the orientations, it induces a natural base $\xi_{r}$ for each $E_{r}^{n}(r \geq 2)$. Now consider the bilinear pairing:

$$
\begin{aligned}
\tau_{r}: & E_{r} \otimes E_{r} \longrightarrow \mathbb{R} \\
\tau_{r}(\varphi \otimes \psi) & =\left\langle\varphi \cdot d_{r} \psi, \xi_{r}\right\rangle \stackrel{\text { def }}{=}\left\langle\varphi, d_{r} \psi\right\rangle .
\end{aligned}
$$

It is easy to verify that

$$
\left\langle\varphi, d_{r} \psi\right\rangle=(-1)^{(\operatorname{deg} \varphi+1)(\operatorname{deg} \psi+1)}\left\langle\psi, d_{r} \varphi\right\rangle .
$$

In our situation $n=4 m-1$. This implies that $\tau_{r}$ restricted to $E_{r}^{2 m-1}$ is symmetric. By viewing $\tau_{r}$ as a symmetric matrix one can take its signature, $\operatorname{sign} \tau_{r}$. Now define

$$
\tau=\sum_{r \geq 2} \operatorname{sign} \tau_{r}
$$

This is clearly a topological invariant.

Theorem 4.4 (Adiabatic limit formula). We have the equality

$$
\tau=\frac{1}{2} \sum_{\lambda_{0}, \lambda_{1}=0} \lim _{x \rightarrow 0} \operatorname{sgn} \lambda_{x} .
$$

Consequently the following adiabatic limit formula holds,

$$
\lim _{x \rightarrow 0} \eta\left(A_{x}\right)=2 \int_{B} \mathscr{L}\left(\frac{R^{B}}{2 \pi}\right) \wedge \tilde{\eta}+\eta\left(A_{B} \otimes \operatorname{ker} A_{Y}\right)+2 \tau .
$$

Proof. It suffices to prove the first equality (see Corollary 4.1). To do this, we claim that

$$
\sum_{\lambda_{0}, \lambda_{1}=0} \frac{1}{2} \lim _{x \rightarrow 0} \operatorname{sgn} \lambda_{x}=\sum_{\lambda_{0}, \lambda_{1}=0}^{\prime} \lim _{x \rightarrow 0} \operatorname{sgn} \lambda_{x}
$$

where $\Sigma^{\prime}$ indicates that the summation is over all $\lambda_{x}$, which are the eigenvalues of $*_{x} d$ on $\Lambda^{2 m-1}$. Granted this, Theorem 4.4 can then be proved as follows. 
Since $E_{2}=H^{*}\left(B, \mathscr{H}^{*}(Y)\right)$, it inherits an inner product $(\cdot, \cdot)$, which then induces an inner product on each $E_{r}$. Define

$$
\left\langle\varphi, d_{r} \psi\right\rangle=\left(\varphi, *_{r} d_{r} \varphi\right)
$$

Then as symmetric mappings, the eigenvalues of $\tau_{r}$ are the same as those of ${ }_{r} d_{r}$. By Theorem 0.2,

$$
d_{r} \sim x^{-r} d \text { and } *_{r} \sim * .
$$

We see immediately that the first statement of Theorem 4.4 holds. The adiabatic limit formula follows then from (0.1).

It remains to justify the claim. For simplicity we suppress the $x$ dependence and consider $A=* d \pm d *: \Lambda^{*}(M) \longrightarrow \Lambda^{*}(M)$. Since $*: \Lambda^{\text {even }} \longrightarrow \Lambda^{\text {odd }}$ is an isomorphism, $A$ is just two copies of its restriction to $\Lambda^{\text {odd }}$. By the Hodge decomposition

$$
\Lambda^{\text {odd }}=\mathscr{H}^{\text {odd }} \oplus d \Lambda^{\text {even }} \oplus \delta \Lambda^{\text {even }} .
$$

Observe that the operator A annihilates $\mathscr{H}^{\text {odd }}$ and coincides up to sign with $d *$ on $d \Lambda^{\text {even }}$ and with $* d$ on $\delta \Lambda^{\text {even }}$.

Now $d *: d \Lambda^{2 \rho} \longrightarrow d \Lambda^{4 k-2 p-2}, * d: \delta \Lambda^{2 p} \longrightarrow \delta \Lambda^{4 m-2 p}$, and $2 p \neq 4 m-$ $2 p-2,2 p=4 m-2 p$ iff $p=m$. Hence we can decompose

$$
A=A_{0} \oplus A_{1} \oplus A_{2}
$$

where $A_{0}$ is the zero operator on $\mathscr{H}^{\text {odd }}$ and $A_{1}=* d$ on $\delta \Lambda^{2 m}$ and $A_{2}$ is an operator of the form

$$
\left(\begin{array}{cc}
0 & T \\
T^{*} & 0
\end{array}\right)
$$

therefore having symmetric spectrum.

We now turn to applications of $(0.1)$ and give intrinsic characterizations of the nonmultiplicativity of signature for manifolds with boundary.

Chern-Hirzebruch-Serre first studied the multiplicative behavior of signature for closed manifolds [CHS]. They showed that for a fibration $Z \longrightarrow N^{n} \longrightarrow B$, we have the multiplicativity

$$
\operatorname{sign} N=\operatorname{sign} B \cdot \operatorname{sign} Z,
$$

provided $Z, B$ are closed manifolds, and $\pi_{1}(B)$ acts trivially on $H^{*}(Z)$. Later Atiyah [A] gave an example showing that this naive sense of multiplicativity does not hold in general. However, he observed that, by the Hirzebruch signature theorem and Atiyah-Singer Family Index Theorem [AS2], one does have a generalized multiplicativity for closed manifolds,

$$
\operatorname{sign} N=\operatorname{sign}(B, \operatorname{Sign} Z)=\int_{B} \mathscr{L}(B) \wedge \operatorname{ch}(\operatorname{Sign} Z),
$$


where $\mathscr{L}(B)$ is a characteristic class that may substitute for the Hirzebruch Lgenus, $\operatorname{Sign} Z$ is the signature bundle of $Z$ over $B$, and $\operatorname{ch}(\operatorname{Sign} Z)$ is its Chern character. When $\pi_{1}(B)$ acts trivially on $H^{*}(Z)$, this reduces to (4.15).

This generalized multiplicativity has a Leray spectral sequence interpretation. Consider the pairing

$$
E_{r}^{i} \otimes E_{r}^{n-i} \longrightarrow \mathbb{R}, \quad \varphi \otimes \psi \longrightarrow\left\langle\varphi \cdot \psi, \xi_{r}\right\rangle,
$$

where $E_{r}$ is the Leray spectral sequence of the fibration and $\xi_{r}$ is defined in the same way as before. When $n=4 k$, the above pairing restricts to a symmetric pairing on $E_{r}^{2 k}$, whose signature is denoted by $\sigma_{r}$. Then (4.16) is equivalent to

$$
\sigma_{2}=\sigma_{\infty} .
$$

This follows from [CHS] and the signature theorem for twisted coefficients [L, AS2].

However, for manifolds with boundary, the generalized multiplicativity fails, as can be seen in the following example.

Example (Disk bundles). Let $V^{2} \longrightarrow B^{2}$ be an oriented 2-plane bundle over an oriented surface. Denote by $\Phi$ the Thom class and $\chi(V)$ the Euler number of V. Consider its disk bundle $D^{2} \longrightarrow D(V) \longrightarrow B^{2}$ with its sphere bundle $S(V)$. By the Thom isomorphism

$$
H^{*-2}(B) \stackrel{\cup \Phi}{\longrightarrow} H^{*}(D(V), S(V)),
$$

one easily finds

$$
\operatorname{sign}(D(V))=\operatorname{sgn} \chi(V) .
$$

But $\operatorname{sign}(B)=\operatorname{sign}\left(D^{2}\right)=0$. In particular, if $\mathrm{V}$ is the Hopf bundle, $\operatorname{sign}(D(V))=1 \neq 0$.

Thus it is quite interesting to study the difference

$$
\Delta \stackrel{\text { def }}{=} \operatorname{sign}(B, \operatorname{Sign} Z)-\operatorname{sign} N=\int_{B} \mathscr{L}(B) \wedge \operatorname{ch}(\operatorname{Sign} Z)-\operatorname{sign} N .
$$

$\Delta$ is called the nonmultiplicativity of signature. We separate our discussion into two cases, depending on how the boundary of the total manifold arises.

Case (a). In the fibration, we assume $B$ is closed, even-dimensional, and $Z$ has nonempty boundary $Y$. Consequently $N$ has a nonempty boundary $M$ that is fibered over $B$ with typical fiber $Y$ :

$$
Y \longrightarrow M \longrightarrow B
$$

By the extended Novikov additivity [BC5] and the generalized multiplicativity for closed manifolds, $\Delta$ depends only on the associated boundary fibration.

Theorem 0.4 (a). The topological invariant $\tau$, which is defined from the closed fibration $Y \longrightarrow M \longrightarrow B$, intrinsically characterizes the nonmultiplicativity of 
signature. That is, whenever there exists another fibration of manifolds with boundary $Z \longrightarrow N^{n} \longrightarrow B$ such that $\partial Z=Y, \partial N=M$, we have $\tau=\Delta$.

Proof. We start with Atiyah-Patodi-Singer's signature formula [APS] for a manifold with boundary,

$$
\operatorname{sign} N=\int_{N} \mathscr{L}\left(\frac{R^{x}}{2 \pi i}\right)-\frac{1}{2} \eta\left(A_{x}\right) .
$$

Take the adiabatic limit of both sides. The left-hand side remains since signature is a topological invariant. The first term on the right-hand side yields, by Lemma 1.1,

$$
\int_{N} \mathscr{L}\left(\frac{R^{B}}{2 \pi i}\right) \wedge \mathscr{L}\left(\frac{R^{Z}}{2 \pi i}\right)=\int_{B} \mathscr{L}\left(\frac{R^{B}}{2 \pi i}\right) \wedge\left(\int_{Z} \mathscr{L}\left(\frac{R^{Z}}{2 \pi i}\right)\right),
$$

while the second term can be replaced by the adiabatic limit formula $(0.1)$. Therefore we obtain

$$
\begin{aligned}
\operatorname{sign}(N)= & \int_{B} \mathscr{L}\left(\frac{R^{B}}{2 \pi i}\right) \wedge\left(\int_{Z} \mathscr{L}\left(\frac{R^{Z}}{2 \pi i}\right)\right) \\
& -\int_{B} \mathscr{L}\left(\frac{R^{B}}{2 \pi i}\right) \wedge \tilde{\eta}-\frac{1}{2} \eta\left(A_{B} \otimes \operatorname{ker} A_{Y}\right)-\tau \\
= & \int_{B} \mathscr{L}\left(\frac{R^{B}}{2 \pi i}\right) \wedge\left(\int_{Z} \mathscr{L}\left(\frac{R^{Z}}{2 \pi i}\right)-\tilde{\eta}\right)-\tau,
\end{aligned}
$$

where the $\eta$-term drops out because $\operatorname{dim} B$ is even. In fact, the involution $\bar{\tau}=(\sqrt{-1})^{p / 2} f_{1} \cdots f_{p}$ anticommutes with $A_{B} \otimes \operatorname{ker} A_{Y}$, which can be seen by using (4.7).

On the other hand, Bismut-Cheeger's Families Index Theorem [BC3, BC4] states that $\int_{Z} \mathscr{L}\left(\frac{R^{Z}}{2 \pi i}\right)-\tilde{\eta}$ is a representative for the chern character of the signature bundle $\operatorname{Sign} Z$. Combining these two finishes the proof.

Case (b). Now assume that $Z$ is closed but $B$ has a nonempty boundary $\partial B$. Again $\Delta$ is intrinsic to the boundary. We show that the same conclusion as in Theorem $0.4(a)$ holds here.

Theorem 0.4(b). Again, $\tau$ intrinsically characterizes the nonmultiplicativity of signature in this case.

Proof. By the same reasoning as in the proof of Theorem 0.4(a), one arrives at:

$$
\begin{aligned}
\operatorname{sign}(N)= & \int_{B} \mathscr{L}\left(\frac{R^{B}}{2 \pi i}\right) \wedge\left(\int_{Z} \mathscr{L}\left(\frac{R^{Z}}{2 \pi i}\right)\right) \\
& -\int_{\partial B} \mathscr{L}\left(\frac{R^{\partial B}}{2 \pi i}\right) \wedge \widetilde{\eta}-\frac{1}{2} \eta\left(A_{\partial B} \otimes \operatorname{Sign} Z\right)-\tau .
\end{aligned}
$$


On the other hand, by the Atiyah-Patodi-Singer index theorem,

$$
\operatorname{sign}(B, \operatorname{Sign} Z)=\int_{B} \mathscr{L}\left(\frac{R^{B}}{2 \pi i}\right) \wedge \operatorname{ch}(\operatorname{Sign} Z)-\frac{1}{2} \eta\left(A_{\partial B} \otimes \operatorname{Sign} Z\right) .
$$

Our claim clearly follows if we show

$$
\begin{aligned}
\int_{B} \mathscr{L}\left(\frac{R^{B}}{2 \pi i}\right) \wedge \operatorname{ch}(\operatorname{Sign} Z) \\
\quad=\int_{B} \mathscr{L}\left(\frac{R^{B}}{2 \pi i}\right) \wedge\left(\int_{Z} \mathscr{L}\left(\frac{R^{Z}}{2 \pi i}\right)\right)-\int_{\partial B} \mathscr{L}\left(\frac{R^{\partial B}}{2 \pi i}\right) \wedge \tilde{\eta}
\end{aligned}
$$

This is a consequence of the transgression formula $(0.2)$. In fact, since $\mathscr{L}\left(\frac{R^{B}}{2 \pi i}\right)$ is a closed form,

$$
\begin{aligned}
\int_{B} \mathscr{L}\left(\frac{R^{B}}{2 \pi i}\right) \wedge\left(\operatorname{ch}(\operatorname{Sign} Z)-\int_{Z} \mathscr{L}\left(\frac{R^{Z}}{2 \pi i}\right)\right) & =-\int_{B} d\left(\mathscr{L}\left(\frac{R^{B}}{2 \pi i}\right) \wedge \tilde{\eta}\right) \\
& =-\int_{\partial B} \mathscr{L}\left(\frac{R^{\partial B}}{2 \pi i}\right) \wedge \tilde{\eta}
\end{aligned}
$$

We finish our discussion with an example. Consider the fibration $Y \rightarrow M \rightarrow$ $B$. Suppose it admits a flat connection, i.e. there is a splitting

$$
T M=T^{V} M \oplus T^{H} M
$$

such that the horizontal distribution $T^{H} M$ is involutive: $\left[T^{H} M, T^{H} M\right] \subset$ $T^{H} M$. We want to show that, in this case, the intrinsic nonmultiplicativity $\tau=0$.

We first do some general calculations using Serre's filtration. Recall that $F^{i}=\left\{\omega \mid \omega \in \Lambda^{r}(M)\right.$, and $\omega\left(Y_{1}, \ldots, Y_{r}\right)=0$ whenever $r-i+1$ of the $r$ tangential vectors are vertical $\}$. In what follows, we use $X$ 's to denote the tangent vectors on $B$ as well as their horizontal lifts and $V$ 's to denote vertical vectors. Now

$$
\begin{aligned}
E_{0}^{i}=F^{i} / F^{i+1} & \rightarrow C^{\infty}\left(\Lambda^{i}(B) \otimes C^{\infty}\left(\Lambda^{*}(Y)\right)\right) \\
{[\omega] } & \rightarrow \widetilde{\omega},
\end{aligned}
$$

where $\widetilde{\omega}\left(X_{1}, \ldots, X_{i}\right)\left(V_{1}, \ldots, V_{j}\right)=\omega\left(X_{1}, \ldots, X_{i}, V_{1}, \ldots, V_{j}\right)$. It is clear that $\widetilde{\omega}$ is independent of the choice of representative. Conversely, given $\widetilde{\omega} \in$ $C^{\infty}\left(\Lambda^{i}(B) \otimes C^{\infty}\left(\Lambda^{*} Y\right)\right)$, we define

$$
\omega\left(X_{1}, \cdots, X_{k}, V_{1}, \cdots, V_{j}\right)= \begin{cases}\widetilde{\omega}\left(X_{1}, \cdots, X_{i}\right)\left(V_{1}, \cdots, V_{j}\right), & \text { if } k=i \\ 0, & \text { otherwise }\end{cases}
$$

Thus, $E_{0}^{i}$ can be identified with $C^{\infty}\left(\Lambda^{i}(B) \otimes C^{\infty}\left(\Lambda^{*} Y\right)\right)$. 
To compute $d_{0}$, we look at the diagram:

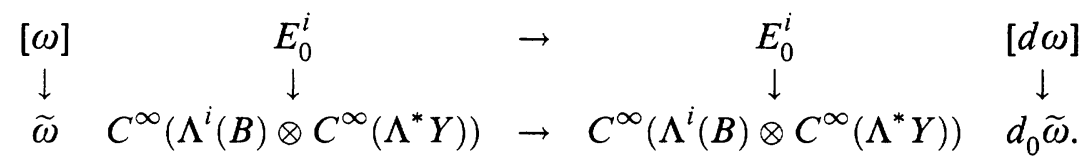

The well-known formula for the exterior derivative gives

$$
\begin{aligned}
d \omega\left(X_{1}, \cdots, X_{i}, V_{1}, \cdots, V_{j+1}\right) \\
=\sum_{k}(-1)^{k} X_{k}\left(\omega\left(X_{1}, \cdots, \widehat{X}_{k}, \cdots, V_{j+1}\right)\right) \\
\quad+\sum_{k}(-1)^{i+k} V_{k}\left(\omega\left(X_{1}, \cdots, \widehat{V}_{k}, \cdots, V_{j+1}\right)\right) \\
\quad+\sum_{k, l}(-1)^{k+l} \omega\left(\left[X_{k}, X_{l}\right], \cdots, \widehat{X}_{k}, \cdots, \widehat{X}_{l}, \cdots, V_{j+1}\right) \\
\quad+\sum_{k, l}(-1)^{k+i+l} \omega\left(\left[X_{k}, V_{l}\right], \cdots, \widehat{X}_{k}, \cdots, \widehat{V}_{l}, \cdots, V_{j+1}\right) \\
\quad+\sum_{k, l}(-1)^{k+l} \omega\left(\left[V_{k}, V_{l}\right], \cdots, \widehat{V}_{k}, \cdots, \widehat{V}_{l}, \cdots, V_{j+1}\right) .
\end{aligned}
$$

By the way we define $\omega$, the first and the third terms drop out. Also $\left[X_{k}, V_{l}\right] \in T^{V} M$. It follows that the fourth term vanishes as well. Thus,

$$
\begin{aligned}
d \omega( & \left.X_{1}, \ldots, V_{j+1}\right) \\
= & \sum_{k}(-1)^{i+k} V_{k}\left(\widetilde{\omega}\left(X_{1}, \ldots, X_{i}\right)\left(V_{1}, \ldots, \widehat{V}_{k}, \ldots, V_{j+1}\right)\right) \\
& +\sum_{k, l} \widetilde{\omega}\left(X_{1}, \ldots, X_{i}\right)\left(\left[V_{k}, V_{l}\right], \ldots, \widehat{V}_{k}, \ldots, \widehat{V}_{l}, \ldots, V_{j+1}\right) \\
= & (-1)^{i} d_{Y}\left(\widetilde{\omega}\left(X_{1}, \ldots, X_{i}\right)\left(V_{1}, \ldots, V_{j+1}\right),\right.
\end{aligned}
$$

where $d_{Y}$ denotes the exterior differentiation along the fiber. This implies $d_{0}=(-1)^{i} d_{Y}$, and hence $E_{1}^{i}$ can be identified with $C^{\infty}\left(\Lambda^{i}(B) \otimes \mathscr{H}^{*}(Y)\right)$, if we denote by $\mathscr{H}^{*}(Y)$ the bundle of the fiber harmonic forms. 
We now proceed to compute $d_{1}$. If $\widetilde{\omega} \in C^{\infty}\left(\Lambda^{i}(B) \otimes \mathscr{H}^{*}(Y)\right)$, then $d \omega \in$ $F^{i+1}$, and

$$
\begin{aligned}
d \omega\left(X_{1}, \ldots, X_{i+1}, V_{1}, \ldots, V_{j}\right) \\
=\sum_{k}(-1)^{k} X_{k}\left(\omega\left(X_{1}, \ldots, \widehat{X}_{k}, \ldots, V_{j}\right)\right) \\
\quad+\sum_{k, l}(-1)^{k+l} \omega\left(\left[X_{k}, X_{l}\right], \ldots, \widehat{X}_{k}, \ldots, \widehat{X}_{l}, \ldots, V_{j}\right) \\
\quad+\sum_{k, l}(-1)^{i+1+k+l} \omega\left(\left[X_{k}, V_{l}\right], \ldots, \widehat{X}_{k}, \ldots, \widehat{V}_{l}, \ldots, V_{j}\right) \\
=\sum_{k}(-1)^{k}\left[X_{k}\left(\omega\left(X_{1}, \ldots, \widehat{X}_{k}, \ldots, V_{j}\right)\right)\right. \\
\left.\quad-\sum_{l} \omega\left(X_{1}, \ldots, \widehat{X}_{k}, \ldots,\left[X_{k}, X_{l}\right], \ldots, V_{j}\right)\right] \\
\quad+\sum_{k, l}(-1)^{k+l} \omega\left(\left[X_{k}, X_{l}\right], \ldots, \widehat{X}_{k}, \ldots, \widehat{X}_{l}, \ldots, V_{j}\right) .
\end{aligned}
$$

Let $\nabla$ be the connection on $T^{V} M$ so that (see $\S 4.1$ where this connection is denoted by $\nabla^{Y}$ )

$$
\nabla_{X_{k}} V_{l}=\left[X_{k}, V_{l}\right]
$$

This connection gives rise to a connection on the infinite-dimensional bundle $C^{\infty}\left(\Lambda^{*} Y\right)$ over $B$, which in turn projects to a connection on $\mathscr{H}^{*}(Y)$; see [BC2] for details. Hence

$$
\begin{aligned}
& X_{k}\left(\omega\left(X_{1}, \ldots, \widehat{X}_{k}, \ldots, V_{j}\right)\right)-\sum_{l} \omega\left(X_{1}, \ldots, \widehat{X}_{k}, \ldots,\left[X_{k}, X_{l}\right], \ldots, V_{j}\right) \\
& =\left(\nabla_{X_{k}} \widetilde{\omega}\left(X_{1}, \ldots, \widehat{X}_{k}, \ldots, X_{i+1}\right)\left(V_{1}, \ldots, V_{j}\right) .\right.
\end{aligned}
$$

From here we conclude that $d_{1}=\pi_{Y} d^{\nabla} \pi_{Y}=d^{\pi_{Y} \nabla \pi_{Y}}$. Here $\pi_{Y}$ denotes the projection of $C^{\infty}\left(\Lambda^{i}(B) \otimes C^{\infty}\left(\Lambda^{*} Y\right)\right)$ onto $C^{\infty}\left(\Lambda^{i} B, \mathscr{H}^{*}(Y)\right)$ induced by the Hodge theorem. Therefore $E_{2}^{i}$ can be identified with $H^{i}\left(B, \mathscr{H}^{*}(Y)\right)$.

Similarly we want to compute $d_{2}$. Let $\widetilde{\omega} \in H^{i}\left(B, \mathscr{H}^{*}(Y)\right)$. In general, we do not have $d \omega \in F^{i+2}$ since $d_{1} \widetilde{\omega}=0$ implies only $\pi_{Y} \widetilde{d \omega}=0$. Thus $\omega$ is not a valid representative for $\widetilde{\omega}$ in $E_{2}$. 
Now this is exactly where our assumption really simplifies the calculation. Under our assumption, $d_{1}=d^{\nabla}$. Further,

$$
\begin{aligned}
& d \omega\left(X_{1}, \ldots, X_{i+2}, V_{1}, \ldots, V_{j-1}\right) \\
& =\sum_{k, l} \omega\left(\left[X_{k}, X_{l}\right], \ldots, \widehat{X}_{k}, \ldots, \widehat{X}_{l}, \ldots, V_{j-1}\right) \\
& =\sum_{k, l}(-1)^{i+k+l} \widetilde{\omega}\left(X_{1}, \ldots, \widehat{X}_{k}, \ldots, \widehat{X}_{l}, \ldots, X_{i+2}\right) \\
& \quad \times\left(\Omega\left(X_{k}, X_{l}\right), V_{1}, \ldots, V_{j-1}\right),
\end{aligned}
$$

where $\Omega$ is the curvature operator.

Given $\widetilde{\omega}$ as above, the formula

$$
\begin{aligned}
& \widetilde{\omega}_{k l}\left(X_{1}, \ldots, X_{i+2}\right)\left(V_{1}, \ldots, V_{j-1}\right) \\
& \quad=\widetilde{\omega}\left(X_{1}, \ldots, \widehat{X}_{k}, \ldots, \widehat{X}_{l}, \ldots, X_{i+2}\right)\left(\Omega\left(X_{k}, X_{l}\right), V_{1}, \ldots, V_{j-1}\right)
\end{aligned}
$$

defines $\widetilde{\omega}_{k l} \in C^{\infty}\left(\Lambda^{i}(B) \otimes C^{\infty}\left(\Lambda^{*} Y\right)\right)$. Let $\pi_{B}$ be the projection of $C^{\infty}\left(\Lambda^{i}(B) \otimes C^{\infty}\left(\Lambda^{*} Y\right)\right)$ onto $H^{i}\left(B, \mathscr{H}^{*}(Y)\right)$ induced by the Hodge theorem. It is clear that

$$
d_{2} \widetilde{\omega}=\sum_{k, l}(-1)^{i+k+l} \pi_{B} \widetilde{\omega}_{k l} .
$$

When $\Omega=0$, we have $\widetilde{\omega}_{k l}=0$ and so $d_{2}=0$. Moreover, it also follows that $d_{r}=0$ for all $r \geq 2$. (This is not true if we just assume $\pi_{B} \widetilde{\omega}_{k l}=0$.) This shows that the spectral sequence degenerates at $E_{2}$.

An interesting special case is when one considers a locally symmetric space $X$ of $\mathbb{Q}$-rank one. The cross section $M$ of a cusp has the fibration structure of a flat torus bundle over a compact locally symmetric space $B$ (cf. [Mü2]). In [Mü2] Müller studied the $L^{2}$-index theory on $X$. Among other things, Müller derived the following formula for the $L^{2}$-signature of $X$ :

$$
L^{2}-\operatorname{sign}(X)=\int_{X} \mathscr{L}-L(0)-1 / 2 \eta,
$$

where $L(0)$ is the value of some $L$-function at 0 and $\eta$ is the twisted eta invariant on $B$, see [Mü2] for details.

On the other hand, if we apply the Atiyah-Patodi-Singer formula to the manifold $Y$, which is obtained from $X$ by chopping off the cusps, and take the adiabatic limit, we have

$$
\operatorname{sign}(Y, \partial Y)=\int_{X} \mathscr{L}-\int_{B} \widehat{A} \wedge \tilde{\eta}-1 / 2 \eta-\tau .
$$

In [BC6] $\int_{B} \widehat{A} \wedge \tilde{\eta}$ is shown to be exactly $L(0)$. And by our above discussion, $\tau=0$. Hence we deduce

$$
L^{2}-\operatorname{sign}(X)=\operatorname{sign}(Y, \partial Y)
$$




\section{ACKNOWLEDGMENTS}

This paper represents part of the author's Ph.D. thesis. He is greatly indebted to his advisor, Jeff Cheeger, whose careful guidance and penetrating insights are indispensable in this work. He would also like to thank Jean-Michel Bismut for helpful comments on an earlier version and Rafe Mazzeo and Richard Melrose for their inspiring work.

\section{REFERENCES}

[A] M. F. Atiyah, The signature of fibre-bundles, Global Analysis (D. G. Spencer and S. Iyanaga, eds.), Princeton Univ. Press, Princeton, NJ, 1969, pp. 73-84.

[ADS] M. F. Atiyah, H. Donnelly, and I. M. Singer, Eta invariants, signature defect of cusps and values of L-functions, Ann. of Math. (2) 118 (1983), 131-177.

[APS] M. F. Atiyah, V. K. Patodi, and I. M. Singer, Spectral asymmetry and Riemannian geometry. I, Math. Proc. Cambridge Philos. Soc. 77 (1975), 43-69.

[AS1] M. F. Atiyah and I. M. Singer, The index of elliptic operators. I, Ann. of Math. 87 (1968), 484-530.

[AS2] _ The index of elliptic operators. III, Ann. of Math. 87 (1968), 546-604.

[AS3] _ The index of elliptic operators. V, Ann. of Math. 93 (1971), 139-149.

[BGV] N. Berline, E. Getzler, and M. Vergne, Heat kernels and Dirac operators, manuscript.

[B] J.-M. Bismut, The Atiyah-Singer index theorem for families of Dirac operators: two heat equation proofs, Invent. Math. 83 (1986), 91-151.

[BC1] J.-M. Bismut and J. Cheeger, Invariants eta et indice des familles pour varietes a bord, C. R. Acad. Sci. Paris Sér. I Math. 305 (1987), 127-130.

[BC2] — $\eta$-invariants and their adiabatic limits, J. Amer. Math. Soc. 2 (1989), 33-70.

[BC3] __ Families index for manifolds with boundary, superconnections and cones. I, J. Funct. Anal. 89 (1990), 313-363.

[BC4] __ Families index for manifolds with boundary, superconnections and cones. II, J. Funct. Anal. 90 (1990), 306-354.

[BC5] _ Remarks on the index theorem for families of Dirac operators on manifolds with boundary (to appear).

[BC6] - in preparation.

[BF1] J.-M. Bismut and D. Freed, The analysis of elliptic families. I, Comm. Math. Phys. 106 (1986), 159-176.

[BF2] _ The analysis of elliptic families. II, Comm. Math. Phys. 107 (1986), 103-163.

[C1] J. Cheeger, Spectral geometry of singular Riemannian spaces, J. Differential Geom. 18 (1983), 575-657.

[C2] - $\eta$-invariants, the adiabatic approximation and conical singularities. J. Differential Geom. 26 (1987), 175-221.

[CD] J. Cheeger and X. Dai, The $L^{2}$-cohomology and signature of a bundle of cones, in preparation.

[CGT] J. Cheeger, M. Gromov, and M. Taylor, Finite propagation speed, kernel estimates for functions of the Laplace operator, and the geometry of complete Riemannian manifolds, J. Differential Geom. 17 (1982), 15-53.

[CHS] S. S. Chern, F. Hirzebruch, and J. P. Serre, On the index of a fibered manifold, Proc. Amer. Math. Soc. 8 (1957), 587-596.

[DM] X. Dai and R. Melrose, Adiabatic limit and analytic torsion, in preparation.

[D] H. Donnelly, Local index theorem for families, Michigan Math. J. 35 (1988), 11-20.

[F] D. Freed, Determinants, torsion and strings, Comm. Math. Phys. 107 (1986), 483-513.

[G] E. Getzler, A short proof of the Atiyah-Singer index theorem, Topology 25 (1986), 111-117. 
[Gi] P. Gilkey, Invariance theory, the heat equation, and the Atiyah-Singer index theorem, Math. Lecture Series, No. 11, Publish or Perish, 1984.

$[\mathrm{H}] \quad$ L. Hörmander, The analysis of linear partial differential operators. Vol. III, Grundlehren Math. Wiss, Band 274, Springer, Berlin, Heidelberg, and New York, 1985.

[K] T. Kato, Perturbation theory for linear operators. Springer-Verlag, 1980.

[LM] H. B. Lawson and M.-L. Michelsohn, Spin geometry, Princeton Math. Series, No 38, Princeton Univ. Press, Princeton, NJ, 1989.

[L] G. Lusztig, Novikov's higher signature theorem and families of elliptic operators, J. Differential Geom. 7 (1972), 229-256.

[MM] R. Mazzeo and R. Melrose, The adiabatic limit, Hodge cohomology and Leray's spectral sequence for a fibration, J. Differential Geom. 31 (1990), 185-213.

[M1] R. Melrose, Pseudodifferential operators on manifolds with corners, preprint.

[M2] - Analysis on manifolds with corners, M.I.T. Notes, 1988.

[M3] _ Transformation of boundary problem, Acta Math. 147 (1981), 149-236.

[Mü1] W. Müller, Signature defects of cusps of Hilbert moldular varieties and values of L-series at $s=1$, J. Differential Geom. 20 (1984), 55-119.

[Mü2] _ Manifolds with cusps of rank one, Lecture Notes in Math., vol. 1244, SpringerVerlag, 1987.

[Q] D. Quillen, Superconnections and the Chern character, Topology 24 (1985), 89-95.

[RS] M. Reed and B. Simon, Methods of modern mathematical physics. IV, Analysis of operators, Academic Press, 1978.

[Sn] B. Simon, Semiclassical analysis of low lying eigenvalues. I, Ann. Inst. Henri Poincaré 38 (1983), 295-307.

[Si] I. M. Singer, The $\eta$-invariant and the index, Mathematical Aspects of String Theory (S. T. Yau, ed.), World Scientific, Singapore, 1987.

[S] M. Stern, $L^{2}$-index theorems on locally symmetric spaces, Invent. Math. 96 (1989), 231282.

[W] E. Witten, Global gravitational anomalies, Comm. Math. Phys. 100 (1985), 197-229.

ABSTRACT. We first prove an adiabatic limit formula for the $\eta$-invariant of a Dirac operator, generalizing the recent work of J.-M. Bismut and J. Cheeger. An essential part of the proof is the study of the spectrum of the Dirac operator in the adiabatic limit. A new contribution arises in the adiabatic limit formula, in the form of a global term coming from the (asymptotically) very small eigenvalues.

We then proceed to show that, for the signature operator, these very small eigenvalues have a purely topological significance. In fact, we show that the Leray spectral sequence can be recast in terms of these very small eigenvalues. This leads to a refined adiabatic limit formula for the signature operator where the global term is identified with a topological invariant, the signature of a certain bilinear form arising from the Leray spectral sequence.

As an interesting application, we give intrinsic characterization of the nonmultiplicativity of signature.

Department of Mathematics, Massachusetts Institute of Technology, Cambridge, MASSACHUSETTS 02139 